Portland State University

PDXScholar

Winter 3-4-2014

\title{
Global Resource Management of Response Surface Methodology
}

Michael Chad Miller

Portland State University

Follow this and additional works at: https://pdxscholar.library.pdx.edu/open_access_etds

Part of the Other Mathematics Commons, and the Statistical Methodology Commons Let us know how access to this document benefits you.

\section{Recommended Citation}

Miller, Michael Chad, "Global Resource Management of Response Surface Methodology" (2014).

Dissertations and Theses. Paper 1621.

https://doi.org/10.15760/etd.1620

This Dissertation is brought to you for free and open access. It has been accepted for inclusion in Dissertations and Theses by an authorized administrator of PDXScholar. Please contact us if we can make this document more accessible: pdxscholar@pdx.edu. 
Global Resource Management of Response Surface Methodology

by

Michael Chad Miller

A dissertation submitted in partial fulfillment of the requirements for the degree of

Doctor of Philosophy

in

Systems Science: Mathematics

Dissertation Committee:

Wayne Wakeland, Chair

Bradford Crain

Jeff Fletcher

Thaddeus Shannon

Christof Teuscher

Portland State University

2014 
(C) 2014 Michael Chad Miller 


\begin{abstract}
Statistical research can be more difficult to plan than other kinds of projects, since the research must adapt as knowledge is gained. This dissertation establishes a formal language and methodology for designing experimental research strategies with limited resources. It is a mathematically rigorous extension of a sequential and adaptive form of statistical research called response surface methodology. It uses sponsor-given information, conditions, and resource constraints to decompose an overall project into individual stages. At each stage, a "parent" decision-maker determines what design of experimentation to do for its stage of research, and adapts to the feedback from that research's potential "children", each of whom deal with a different possible state of knowledge resulting from the experimentation of the "parent". The research of this dissertation extends the real-world rigor of the statistical field of design of experiments to develop an deterministic, adaptive algorithm that produces deterministically generated, reproducible, testable, defendable, adaptive, resourceconstrained multi-stage experimental schedules without having to spend physical resource.
\end{abstract}




\section{Acknowledgements}

I would like to my committee for their assistance: Wayne Wakeland, Bradford Crain, Jeff Fletcher, Thaddeus Shannon, and Christof Teuscher. Thank you for your assistance with my academic process regarding the development of my dissertation.

I would also like to thank Darcy Kramer for her work in helping me navigate the social and bureaucratic issues that are involved in this kind of academic endeavor.

In addition, I would also like to thank Eric Thomas, who helped me make my technical language clear and concise for my target audience.

Finally, I would like to thank my father for his support, which has been invaluable to me. 


\section{Table of Contents}

Abstract

Acknowledgements

List of Tables viii

List of Figures $\quad$ XV

1 Introduction 1

1.1 Overall Purpose of Research . . . . . . . . . . . . . . . 1

1.2 Designing Experiments to Acquire Knowledge . . . . . . . . . . . 3

1.3 Managing the Resources Needed to Conduct Experiments . . . . . . . 4

1.4 Approaching Response Surface Methodology With Mathematical and Systems Perspectives ................ 6

2 Background/Related Works 11

2.1 Response Surface Methodology . . . . . . . . . . . . . . 11 
2.1.1 What is Response Surface Methodology? . . . . . . . . . 11

2.1.2 Design of Experiments . . . . . . . . . . . . . . 13

2.1 .3 Automating ARC-RSM . . . . . . . . . . . . . . . 14

2.2 Systemic Representation . . . . . . . . . . . . . . . . 21

2.2.1 Resource-Constrained Project Scheduling . . . . . . . . . . 21

2.2.2 Game Theory/Decision Theory . . . . . . . . . . . 22

3 Introduction to Adaptive Resource-Constrained Response Surface Methodology (ARC-RSM) 27

3.1 Generating approaches to RSM from collections of experimental designs 27

3.2 Response Surface Methodology Iterations in Terms of Collective Behavior 28

3.3 Research Strategy Requirements . . . . . . . . . . . . . . . . 30

3.4 Preference Function Lists . . . . . . . . . . . . . . . . . . . . 34

3.5 General Algorithm for Constructing Research Strategies . . . . . . . 37

4 Creation of research strategies in ARC-RSM 39

4.1 ARC-RSM Algorithm Overview . . . . . . . . . . . . 39

4.2 Basic ARC-RSM Analytical Task Terms . . . . . . . . . . . 42

4.3 Basic ARC-RSM Experimental Design Structures . . . . . . . . . 44

4.4 Ordering of Analytical Tasks . . . . . . . . . . . . . . 46

4.5 Ordering of Experimental Designs . . . . . . . . . . . 47

4.6 Basic Requirements for ARC-RSM Structures . . . . . . . . . 49

4.7 Research Strategy Design Processes . . . . . . . . . . . . . 51 
4.8 ARC-RSM Partial Orders $\ldots \ldots \ldots \ldots \ldots \ldots$

5 Sponsor-Designer specification of ARC-RSM 64

5.1 Preliminary Sponsor-Designer Discussions _.......... . 64

5.2 Sponsor-Designer Task and Design Selections . . . . . . . . . . 66 66

5.3 Sponsor-Designer Feedback Specifications . . . . . . . . . . . . 67

6 Applications and Demonstrations 70

6.1 Summary of Simulation Code and Testing Methods . . . . . . . 70

6.2 Phase 1 - Initial Phase $\ldots \ldots \ldots \ldots \ldots \ldots$

6.2.1 Preliminary Sponsor-Designer Discussions _. . . . . . . 74

6.2.2 Sponsor-Designer Task and Design Selections . . . . . . . . 76

6.2.3 Sponsor-Designer Feedback Specifications . . . . . . . . . 78

6.2.4 Specifications Expressed Formally by Designer for Phase 1 . . 80

6.2.5 Constructed Research Strategy - Initial Phase . . . . . . . 83 83

6.2.6 Model Approximation Testing - Initial Phase . . . . . . . . . 92

6.3 Phase 2 - Minimal Strategy Phase . . . . . . . . . . . . . . . 107

6.3.1 Preliminary Sponsor-Designer Discussions . . . . . . . . 107

6.3.2 Specifications Expressed Formally by Designer for Phase 2 . . 107

6.3.3 Model Approximation Testing - Minimal Strategy Phase . . . 110

6.3.4 Model Approximation Testing - Minimal Strategy Phase . . . 113

6.4 Phase 3 - No Task Compromise Minimal Strategy Phase . . . . . . . 123

6.4.1 Preliminary Sponsor-Designer Discussions . . . . . . . . . 123 


\section{TABLE OF CONTENTS}

6.4.2 Specifications Expressed Formally by Designer for Phase 3 . . 123

6.4.3 Constructed Research Strategy - No Task Compromise Minimal Strategy Phase . . . . . . . . . . . . . . . 125

6.4.4 Model Approximation Testing - No Task Compromise Minimal Strategy Phase . . . . . . . . . . . . . . . 135

6.5 Phase 4 - Logic Error Phase . . . . . . . . . . . . . . . . . . . 145

6.5.1 Preliminary Sponsor-Designer Discussions . . . . . . . . 145

6.5.2 Specifications Expressed Formally by Designer for Phase 4 . . 145

6.5.3 Constructed Research Strategy - Logic Error Phase . . . . . . 147

6.5.4 Model Approximation Testing - Logic Error Phase . . . . . . . 156

6.6 Phase 5 - Catastrophe Phase . . . . . . . . . . . . . 166

6.6.1 Preliminary Sponsor-Designer Discussions . . . . . . . . 166

6.6.2 Specifications Expressed Formally by Designer for Phase 5 . . 166

6.6.3 Constructed Research Strategy - Catastrophe Phase . . . . . . 168

6.6.4 Model Approximation Testing - Catastrophe Phase . . . . . . 177

7 Summary

7.1 Results . . . . . . . . . . . . . . . . . . . . . . . . . 189

7.2 Current Limitations . . . . . . . . . . . . . . . . . . . . . . . . . . 191

7.3 Future Research . . . . . . . . . . . . . . . . . . . . . . . . 192

Bibliography

194 
A Additional Background 213

A.1 Design of Experiments . . . . . . . . . . . . . . . 213

A.2 Set Theory. . . . . . . . . . . . . . . 216

\begin{tabular}{ll} 
Index & 219 \\
\hline
\end{tabular} 


\section{List of Tables}

1.1 Feature comparison of different approaches to Response Surface Methodology. . . . . . . . . . . . . . . . . . . 2

1.2 Steps to complete research. . . . . . . . . . . . . . . . . . 9 9

6.1 Descriptions of the knowledge state index i0 during Phase 1 . . . 84

6.2 Descriptions of the knowledge state index i0x2 during Phase $1 \ldots$. . 85

6.3 Descriptions of the knowledge state index i0x3 during Phase 1 . . . 86

6.4 Descriptions of the knowledge state index i0x4 during Phase $1 \ldots$. . 87

6.5 Descriptions of the knowledge state index i0x5 during Phase 1 . . . 88

6.6 Descriptions of the knowledge state index i0x6 during Phase 1 . . . 89

6.7 Descriptions of the knowledge state index i0x7 during Phase 1 . . . 90

6.8 Descriptions of the knowledge state index i0x8 during Phase 1 . . . 91

6.9 Descriptions of the knowledge state index during Move 1 of Phase 1 -

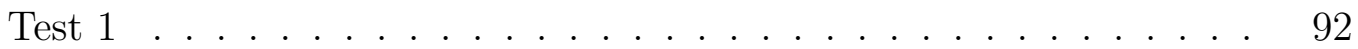




\section{LIST OF TABLES}

6.10 Descriptions of the knowledge state index during Move 2 of Phase 1 Test $1 \ldots \ldots \ldots \ldots$

6.11 Descriptions of the knowledge state index during Move 3 of Phase 1 Test $1 \ldots \ldots \ldots \ldots \ldots$

6.12 Final Results of Phase 1 - Test 1 . . . . . . . . . . . . . 95

6.13 Descriptions of the knowledge state index during Move 1 of Phase 1 Test $2 \ldots \ldots \ldots \ldots$

6.14 Descriptions of the knowledge state index during Move 2 of Phase 1 Test $2 \ldots \ldots \ldots \ldots \ldots$

6.15 Descriptions of the knowledge state index during Move 3 of Phase 1 Test $2 \ldots \ldots \ldots \ldots$

6.16 Final Results of Phase 1 - Test $2 \ldots \ldots$. . . . . . . . . 99

6.17 Descriptions of the knowledge state index during Move 1 of Phase 1 Test $3 \ldots \ldots \ldots \ldots \ldots$

6.18 Descriptions of the knowledge state index during Move 2 of Phase 1 Test $3 \ldots \ldots \ldots \ldots \ldots 10 \ldots \ldots$

6.19 Descriptions of the knowledge state index during Move 3 of Phase 1 Test $3 \ldots \ldots \ldots \ldots$

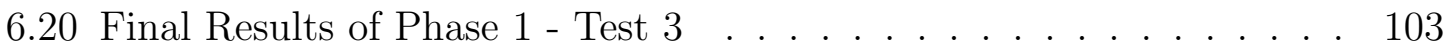

6.21 Descriptions of the knowledge state index during Move 1 of Phase 1 Test $4 \ldots \ldots \ldots \ldots \ldots$. . . . . . . . . . . . . . . . . . . . . . 


\section{LIST OF TABLES}

6.22 Descriptions of the knowledge state index during Move 2 of Phase 1 Test $4 \ldots \ldots \ldots \ldots \ldots$

6.23 Final Results of Phase 1 - Test 4 . . . . . . . . . . . . . . . 106

6.24 Descriptions of the knowledge state index i0 during Phase $2 \ldots \ldots$

6.25 Descriptions of the knowledge state index i0x2 during Phase 2 . . . 112

6.26 Descriptions of the knowledge state index during Move 1 of Phase 2 Test $1 \ldots \ldots \ldots \ldots \ldots \ldots$

6.27 Descriptions of the knowledge state index during Move 2 of Phase 2 Test $1 \ldots \ldots \ldots \ldots$. . . . . . . . . . . . . . . . . . . . . . .

6.28 Descriptions of the knowledge state index during Move 3 of Phase 2 Test $1 \ldots \ldots \ldots \ldots \ldots \ldots$

6.29 Final Results of Phase 2 - Test $1 \ldots \ldots$. . . . . . . . . 116

6.30 Descriptions of the knowledge state index during Move 1 of Phase 2 -

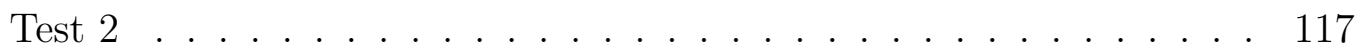

6.31 Final Results of Phase 2 - Test $2 \ldots \ldots$. . . . . . . . . 118

6.32 Descriptions of the knowledge state index during Move 1 of Phase 2 Test $3 \ldots \ldots \ldots \ldots \ldots \ldots$

6.33 Final Results of Phase 2 - Test 3 . . . . . . . . . . . . . 120

6.34 Descriptions of the knowledge state index during Move 1 of Phase 2 Test $4 \ldots \ldots \ldots \ldots \ldots \ldots \ldots$

6.35 Final Results of Phase 2 - Test $4 \ldots \ldots$. . . . . . . . . 122

6.36 Descriptions of the knowledge state index i0 during Phase 3 . . . . . 127 


\section{LIST OF TABLES}

6.37 Descriptions of the knowledge state index i0x2 during Phase $3 \ldots$. . 128

6.38 Descriptions of the knowledge state index i0x3 during Phase 3 . . . . 129

6.39 Descriptions of the knowledge state index i0x4 during Phase 3 . . . . 130

6.40 Descriptions of the knowledge state index i0x5 during Phase 3 . . . 131

6.41 Descriptions of the knowledge state index i0x6 during Phase 3 . . . . 132

6.42 Descriptions of the knowledge state index i0x7 during Phase 3 . . . 133

6.43 Descriptions of the knowledge state index i0x8 during Phase 3 . . . . 134

6.44 Descriptions of the knowledge state index during Move 1 of Phase 3 Test $1 \ldots \ldots \ldots \ldots \ldots \ldots$

6.45 Descriptions of the knowledge state index during Move 2 of Phase 3 Test $1 \ldots \ldots \ldots \ldots \ldots \ldots \ldots$

6.46 Descriptions of the knowledge state index during Move 3 of Phase 3 Test $1 \ldots \ldots \ldots$

6.47 Final Results of Phase 3 - Test 1 . . . . . . . . . . . . . . . . 138

6.48 Descriptions of the knowledge state index during Move 1 of Phase 3 Test $2 \ldots \ldots \ldots \ldots \ldots$

6.49 Final Results of Phase 3 - Test 2 . . . . . . . . . . . . . . . 140

6.50 Descriptions of the knowledge state index during Move 1 of Phase 3 Test $3 \ldots \ldots \ldots \ldots \ldots$. . . . . . . . . . . . . . . . . . . . .

6.51 Final Results of Phase 3 - Test 3 . . . . . . . . . . . . . . . . 142

6.52 Descriptions of the knowledge state index during Move 1 of Phase 3 Test $4 \ldots \ldots \ldots \ldots \ldots \ldots$ 


\section{LIST OF TABLES}

6.53 Final Results of Phase 3 - Test 4 . . . . . . . . . . . . . 144

6.54 Descriptions of the knowledge state index i0 during Phase $4 \ldots \ldots$

6.55 Descriptions of the knowledge state index i0x2 during Phase $4 \ldots \ldots$

6.56 Descriptions of the knowledge state index i0x3 during Phase $4 \ldots \ldots$

6.57 Descriptions of the knowledge state index i0x4 during Phase $4 \ldots \ldots$

6.58 Descriptions of the knowledge state index i0x5 during Phase $4 \ldots \ldots$

6.59 Descriptions of the knowledge state index i0x6 during Phase $4 \ldots \ldots$

6.60 Descriptions of the knowledge state index i0x7 during Phase $4 \ldots \ldots$

6.61 Descriptions of the knowledge state index i0x8 during Phase $4 \ldots \ldots$

6.62 Descriptions of the knowledge state index during Move 1 of Phase 4 Test $1 \ldots \ldots \ldots \ldots \ldots \ldots \ldots$

6.63 Descriptions of the knowledge state index during Move 2 of Phase 4 Test $1 \ldots \ldots \ldots \ldots \ldots \ldots \ldots \ldots$

6.64 Descriptions of the knowledge state index during Move 3 of Phase 4 Test $1 \ldots \ldots \ldots \ldots \ldots \ldots \ldots$

6.65 Final Results of Phase $4-$ Test $1 \ldots \ldots \ldots \ldots$

6.66 Descriptions of the knowledge state index during Move 1 of Phase 4 -

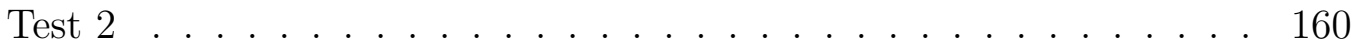

6.67 Final Results of Phase 4 - Test $2 \ldots \ldots \ldots \ldots 16 \ldots$

6.68 Descriptions of the knowledge state index during Move 1 of Phase 4 -

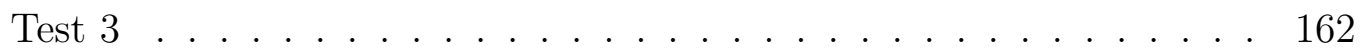

6.69 Final Results of Phase 4 - Test 3 . . . . . . . . . . . . 163 


\section{LIST OF TABLES}

6.70 Descriptions of the knowledge state index during Move 1 of Phase 4 Test $4 \ldots \ldots \ldots \ldots$. . . . . . . . . . . . . . . . . . . . . . . . . .

6.71 Final Results of Phase 4 - Test $4 \ldots \ldots$. . . . . . . . . . 165

6.72 Descriptions of the knowledge state index i0 during Phase 5(pre-catastrophe) 169

6.73 Descriptions of the knowledge state index i0x2 during Phase 5(precatastrophe) ..................... . . . . 170

6.74 Descriptions of the knowledge state index i0x3 during Phase 5(precatastrophe) .......................... 171

6.75 Descriptions of the knowledge state index i0x4 during Phase 5(precatastrophe) . . . . . . . . . . . . . . . 172

6.76 Descriptions of the knowledge state index i0x5 during Phase 5(precatastrophe) ..................... . . . 173

6.77 Descriptions of the knowledge state index i0x6 during Phase 5(precatastrophe) ......................... 174

6.78 Descriptions of the knowledge state index i0x7 during Phase 5(precatastrophe) . . . . . . . . . . . . . . . . 175

6.79 Descriptions of the knowledge state index i0x8 during Phase 5(precatastrophe) ...................... 176

6.80 Descriptions of the knowledge state index during Move 1 of Phase 5 Test $1 \ldots \ldots \ldots \ldots$. . . . . . . . . . . . . . . . . . . . . . . .

6.81 Descriptions of the knowledge state index during Move 3 of Phase 5 Test $1 \ldots \ldots \ldots \ldots \ldots \ldots$ 


\section{LIST OF TABLES}

6.82 Descriptions of the knowledge state index during Move 5 of Phase 5 Test $1 \ldots \ldots \ldots \ldots \ldots$. . . . . . . . . . . . . . . . . . . . . .

6.83 Final Results of Phase 5 - Test 1 . . . . . . . . . . . . . . . 180

6.84 Descriptions of the knowledge state index during Move 1 of Phase 5 Test $1 \ldots \ldots \ldots \ldots \ldots 18 \ldots \ldots$

6.85 Descriptions of the knowledge state index during Move 3 of Phase 5 Test $1 \ldots \ldots \ldots \ldots \ldots$

6.86 Descriptions of the knowledge state index during Move 5 of Phase 5 Test $1 \ldots \ldots \ldots \ldots \ldots$

6.87 Final Results of Phase 5 - Test $1 \ldots \ldots$. . . . . . . . . . . 184

6.88 Descriptions of the knowledge state index during Move 1 of Phase 5 Test $1 \ldots \ldots \ldots \ldots \ldots$

6.89 Descriptions of the knowledge state index during Move 3 of Phase 5 Test $1 \ldots \ldots \ldots \ldots \ldots$

6.90 Descriptions of the knowledge state index during Move 5 of Phase 5 Test $1 \ldots \ldots \ldots \ldots \ldots$. . . . . . . . . . . . . . . . . . .

6.91 Final Results of Phase 5 - Test $1 \ldots \ldots$. . . . . . . . . 188 


\section{List of Figures}

1.1 Designer specifying a machine; from "Mechanisms Of Intelligence" (20) . 6

1.2 Visual demonstration of a sponsor giving the designer a goal through channel 'B'; from "Mechanisms Of Intelligence" (20). This will be used later to help visually present this dissertation's methodology. . . . . . 7

1.3 Goal-directed regulator from "Mechanisms Of Intelligence", where 'C' is the channel the designer uses to communicate with ' $\mathrm{F}$ ', $\left(x_{1}, \ldots, x_{m}\right)$ are the machine inputs, and $\left(y_{1}, \ldots, y_{n}\right)$ are the machine outputs.(20). This is to give a visual hint as to how Ashby's goal-directed regulator is applicable to design of experiments. . . . . . . . . . . . . . 7

1.4 General model of a Design of Experiments system from "Design and analysis of experiments" $(62) . \ldots \ldots \ldots$

2.1 Visualization from Box and Draper (12) of the CDEA process . . . 12 


\section{LIST OF FIGURES}

2.2 Framework for an Automated Response Surface Methodology Algorithm from Neddermeijer, van Oortmarssen, Piersma, and Dekker (66), to show a current attempt to automate response surface methodology. 16

2.3 Diagram from Brown and Schamburg (15) describing their "Modified Response Surface Methodology", to show a different kind of attempt to automate response surface methodology. . . . . . . . . . . . 18

2.4 Diagram from Nicolai and Dekker (68) describing their response surface methodology framework, to show another previous attempt to automate response surface methodology. . . . . . . . . . . . 20

2.5 Structure of the resource-constrained project scheduling problem, where the resources are labeled as $R_{1}$ through $R_{4}$, from Ben Abdelaziz, Krichen,

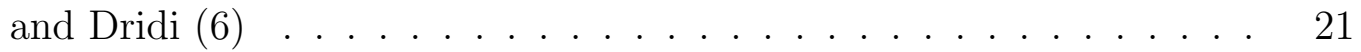

2.6 Initial collection of considered choices a decision maker has available. 24

2.7 Current choices a decision maker has considered available, after unaffordable choices are removed. . . . . . . . . . . . . 25

2.8 Current choices a decision maker has available, after choices with uninteresting results are removed. . . . . . . . . . . . . 25

2.9 Remaining choices a decision maker has available, after choices that do not incorporate the desired standards of correctness are removed. . . 26

3.1 Visual description of a single CDEA iteration of response surface method-

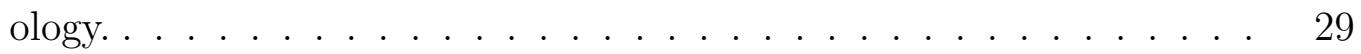

3.2 Visual description of a research strategy. . . . . . . . . . 30 


\section{LIST OF FIGURES}

3.3 Black-box diagram of the generation of a research strategy. . . . . . . 36

3.4 Visual example of acceptable choices. . . . . . . . . . . . . 37

4.1 Visual representation of the algorithm for the construction of a planned move. ............................ 41

6.1 Exit burrs observed during drilling. . . . . . . . . . . . . 71

6.2 Tree graph of research strategy for initial phase. . . . . . . . . . 83

6.3 Tree graph of research strategy for minimal strategy phase. . . . . . . 110

6.4 Tree graph of research strategy for no task compromise minimal strategy phase. . . . . . . . . . . . . . . . 126

6.5 Tree graph of research strategy for logic error phase. . . . . . . . . . . 147

6.6 Tree graph of research strategy (pre-catastrophe) for catastrophe phase.168 


\section{1}

\section{Introduction}

\subsection{Overall Purpose of Research}

The key purpose of this research is to develop a statistical decision-making methodology that can help the researcher to make experimental design choices so as not to waste resources. Shown below is a comparison table describing the difference between how response surface methodology has traditionally been done (Traditional RSM), how others have attempted to automate response surface methodology (Automated RSM), and this new version of response surface methodology (ARC-RSM). 


\subsection{Overall Purpose of Research}

\begin{tabular}{|c|c|c|c|}
\hline Feature & Traditional RSM & Automated RSM & ARC-RSM \\
\hline $\begin{array}{l}\text { Sequential, adaptive } \\
\text { experimentation }\end{array}$ & Yes & Yes & Yes \\
\hline $\begin{array}{l}\text { Able to incorporate } \\
\text { new designs and } \\
\text { methods }\end{array}$ & Yes & No & Yes \\
\hline $\begin{array}{l}\text { Able to directly } \\
\text { compare different } \\
\text { approaches to } \\
\text { Response Surface } \\
\text { Methodology }\end{array}$ & Component-wise & No & Yes \\
\hline $\begin{array}{l}\text { Able to define } \\
\text { current state of } \\
\text { research }\end{array}$ & Limited & Yes & Yes \\
\hline $\begin{array}{l}\text { Able to ensure } \\
\text { terminability }\end{array}$ & No & No & Yes \\
\hline $\begin{array}{l}\text { Overall resource } \\
\text { management }\end{array}$ & No & No & Yes \\
\hline
\end{tabular}

Table 1.1: Feature comparison of different approaches to Response Surface Methodology.

The main contributions of this research is a decision-making statistical approach, based on response surface methodology, which can not only describe what is being done at each stage of research, but can also explain why a particular overall strategy was chosen, and do so in a way that is tenable, comparable, and replicable. 


\subsection{Designing Experiments to Acquire Knowledge}

\subsection{Designing Experiments to Acquire Knowledge}

The general idea of response surface methodology is based around the goal of determining what controllable factors, called predictors, have a significant effect on a main element of interest, called a response, and in what way. In order to do this, there are many questions whose answers are often simply left up to the experimental designer. Here are some of those questions, paraphrased from Box and Draper (12):

1. What input variables should be studied?

2. What kind of relationships should be considered?

3. How should the response be measured?

4. At which levels (values) of a given input variable should experiments be run?

5. How complex must the model be in a particular situation?

6. How should we represent factors which are categorical (types) rather than numbers?

7. What experimental arrangement (design) should be used?

Box and Draper further describe the problems as follows:

"In brief then, the investigator deals with a number of entities whose natures are necessarily matters of opinion. Among these are (a) the identity of the space of the inputs and outputs in which the experiments should be 


\subsection{Managing the Resources Needed to Conduct Experiments}

conducted; (b) the scales, metrics, and transformations in which the variables should be measured; and (c) the location of the region of interest, the specification of the model over it, and the experimental arrangement that should be used to explore the region of interest."

It is very difficult to know the answers to all of the questions above at the start of a project. However, when intelligently used, the sequential and iterative nature of response surface methodology can help to assure that initial bad choices by the experimenter will be corrected as the process proceeds. The effectiveness of response surface methodology strongly depends on the communication between the experimental designer and the experimental designer's sponsor. The present research creates a rigorous methodology, made up of mathematical, statistical, and systems structures, to help researchers take better advantage of response surface methodology as they investigate phenomena and create statistical models.

\subsection{Managing the Resources Needed to Conduct Experiments}

Although individual experimental designs can be adjusted to attempt to get as much information as possible from a current state of knowledge, and experimental design includes resource management at a local level, the iterative process of response surface methodology lacks methods for managing and optimizing the resources re- 


\subsection{Managing the Resources Needed to Conduct Experiments}

quired over an entire project. There are several additional questions related to overall project management:

1. How can a knowledge discovery project be decomposed into a sequence of experimental designs?

2. What requirements must be fulfilled to transition from one stage of research to the next?

3. How might the results of a specific experimental design within a larger project affect the requirements for the rest of the project?

4. How can limited resources be allocated over a series of experimental designs when the results of one experimental design will affect the kind of experimental design to be run next, especially given the fact that the cost of an experimental design will affect what can be allocated for future experimental designs (the nature of which is not yet known)?

5. Under what conditions can a research project be considered efficent and sufficent?

There are already multiple approaches to answering questions similar to these, but they require some form of probabilistic foundation (using probabilities rather than direct statistical evaluation, inference, etc.), and response surface methodology is intended for situations where a probabilistic foundation may not exist during the course of experimentation. Therefore, new approaches must be invented in order to address these questions. This dissertation will provide such an approach. 


\subsection{Approaching Response Surface Methodology With Mathematical}

and Systems Perspectives

\subsection{Approaching Response Surface Methodology With Mathematical and Systems Perspectives}

Response surface methodology uses design of experiments and model conjecture in an informal and sequential knowledge discovery process, using polynomials to approximate the relationship between the value of an output variable (response) and the input variables of interest (predictors). Unfortunately, both of these require considerable interpretation by the researcher. Consequently, while response surface methodology has some formal decision-making criteria, it is incomplete, lacking formal criteria for global resource management and related issues.

An experimental designer works by determining how the testing environment should be configured, which is a concept that Ashby (20) has discussed in "Mechanisms Of Intelligence" (20). Figure 1.1 provides a visual example from Ashby of a designer entering input into a machine:

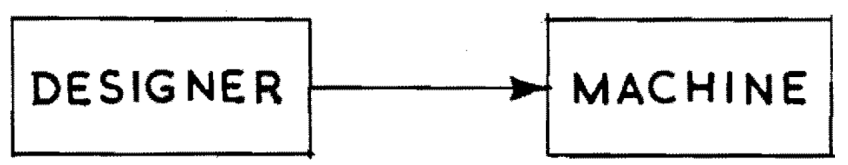

Figure 1.1: Designer specifying a machine; from "Mechanisms Of Intelligence" (20).

The experimental designer gets a goal from a sponsor, which here is based on what the sponsor wishes to learn about the system under investigation. In Ashby's terms, the sponsor has a goal which must be communicated to the designer through 


\subsection{Approaching Response Surface Methodology With Mathematical and Systems Perspectives}

channel 'B'. This concept is described in "Mechanisms Of Intelligence" (20). Figure 1.2 provides a visual example from Ashby of a sponsor giving the designer a goal:

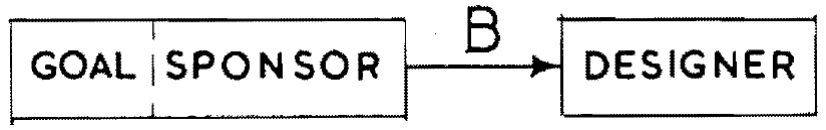

Figure 1.2: Visual demonstration of a sponsor giving the designer a goal through channel 'B'; from "Mechanisms Of Intelligence" (20). This will be used later to help visually present this dissertation's methodology.

The sponsor regulates the designer by transmitting sufficent information to achieve the goal, while the designer can request such information from the sponsor as needed. In experimental design, this is achieved by having the designer interview the sponsor.

Figure 1.3 shows the designer using the goal to configure the machine 'F' (using channel ' $\mathrm{C}$ ') to take in input (information about what is to be regulated) and send out the relevant output (orders for what is to be regulated):

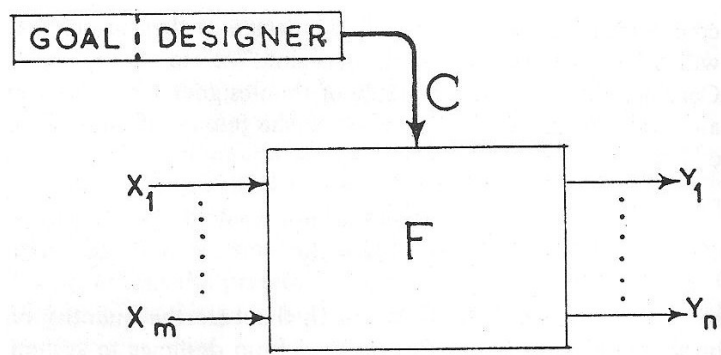

Figure 1.3: Goal-directed regulator from "Mechanisms Of Intelligence", where ' $C$ ' is the channel the designer uses to communicate with ' $F$ ', $\left(x_{1}, \ldots, x_{m}\right)$ are the machine inputs, and $\left(y_{1}, \ldots, y_{n}\right)$ are the machine outputs.(20). This is to give a visual hint as to how Ashby's goal-directed regulator is applicable to design of experiments.

Similarly, Figure 1.4 shows a visual example of a general model of an experimental 


\subsection{Approaching Response Surface Methodology With Mathematical and Systems Perspectives}

design:

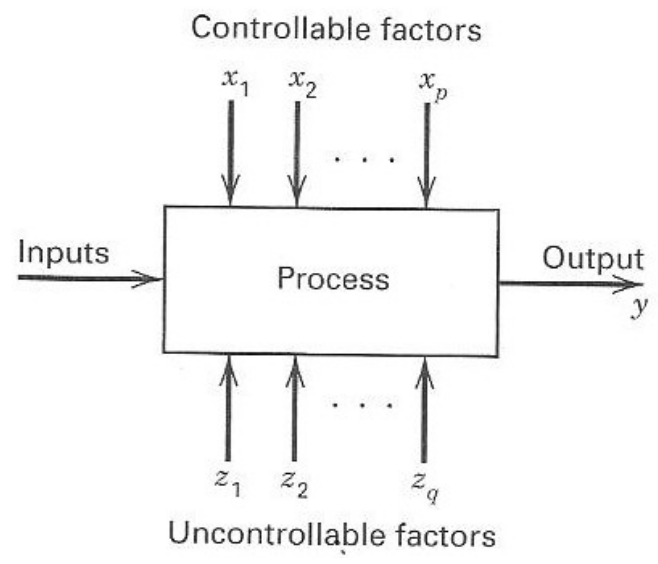

Figure 1.4: General model of a Design of Experiments system from "Design and analysis of experiments" (62).

This is a basic example of the strength of the conceptual relations between Ashby's work on setting goals in cybernetic systems and response surface methodology. In later sections, it will be shown how Ashby's goal-directed regulator will be interpreted in order to govern response surface methodology.

Table 1.2 summarizes the primary deliverables of this research. 


\subsection{Approaching Response Surface Methodology With Mathematical and Systems Perspectives}

\begin{tabular}{|c|}
\hline $\begin{array}{l}\text { Formal language and definitions of what knowledge the sponsor will } \\
\text { contribute, and will receive }\end{array}$ \\
\hline $\begin{array}{l}\text { Formal language and definitions of what knowledge the } \\
\text { experimental designer will contribute, and will receive }\end{array}$ \\
\hline $\begin{array}{l}\text { Formal language and definitions for analytical tasks and } \\
\text { experimental designs }\end{array}$ \\
\hline $\begin{array}{l}\text { Formal language and definitions for preferences and dependencies } \\
\text { of analytical tasks and experimental designs }\end{array}$ \\
\hline Methodology for representing feedback \\
\hline $\begin{array}{l}\text { Methodology for incorporating feedback, preferences and } \\
\text { dependencies into selection of choices for analytical tasks and } \\
\text { experimental designs }\end{array}$ \\
\hline $\begin{array}{l}\text { Rules for what the sponsor and experimental designer are allowed } \\
\text { to influence }\end{array}$ \\
\hline Proofs of unique choice selection by algorithm \\
\hline Proofs that the general algorithm terminates in finite time \\
\hline $\begin{array}{l}\text { Demonstrations of methodology's effectiveness at determining } \\
\text { affordability }\end{array}$ \\
\hline
\end{tabular}

Table 1.2: Steps to complete research.

The remainder of this document further elaborates on these concepts. Chapter 2 describes the background that explains the relevant concepts as they are currently defined. Chapter 3 describes the conceptual model of this methodology's approach to RSM. Chapter 4 contains the descriptions of all the technical components of the ARC-RSM algorithm, with conditions, requirements, and mathematical proofs of conceptual model. Chapter 5 describes how the sponsor and designer interact to give 


\subsection{Approaching Response Surface Methodology With Mathematical and Systems Perspectives}

the designer the necessary information to specify the algorithm's parameters. Chapter 6 describes five interconnected examples showing the applicability, testability, and adaptability of the methodology. Chapter 7 summarizes the results, discusses current limitations in the methodology, and lists some possible future research involving this methodology. 
2

\section{Background/Related Works}

\subsection{Response Surface Methodology}

\subsubsection{What is Response Surface Methodology?}

Response Surface Methodology (RSM) is an iterative process that was invented in 1951 (Box and Wilson (13)), which uses various methods including design of experiments, to determine which controllable factors significantly affect a variable of interest (and how), and then to perform local approximation within the experimental region. In modular form, the most basic procedure in response surface methodology is Conjecture/Design/Experiment/Analyze (CDEA), which can be iterated as necessary, either to explore the current experimental region or to adjust the experimental region as is suitable and accessible. A visualization of this process (Box and Draper (12)) is shown in Figure 2.1. 


\subsection{Response Surface Methodology}

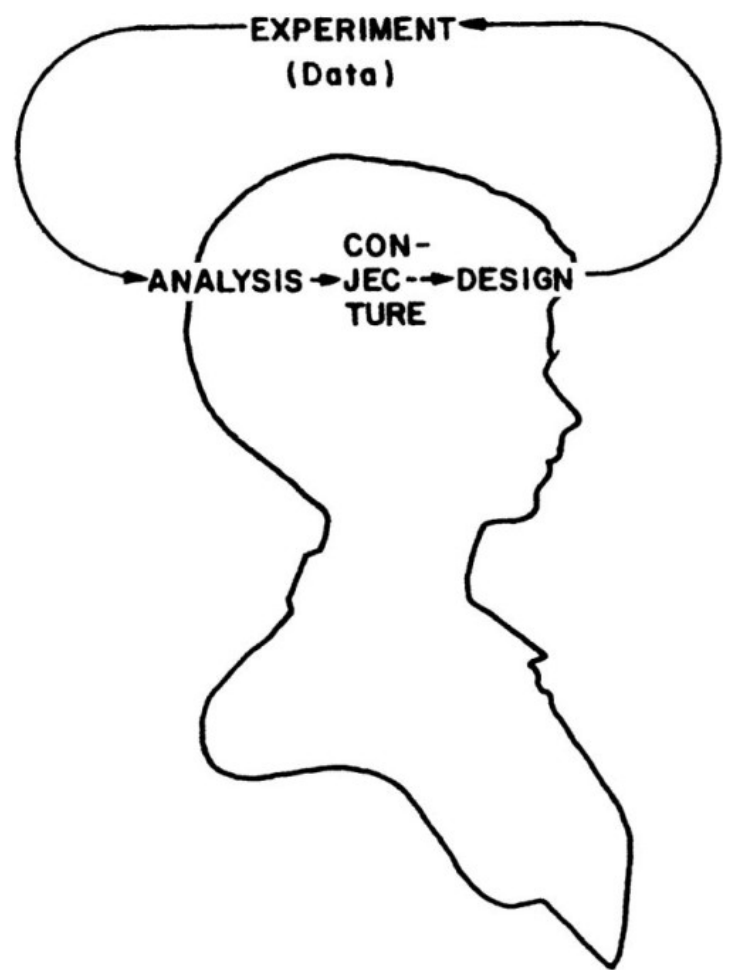

Figure 2.1: Visualization from Box and Draper (12) of the Conjecture/Design/Experiment/Analyze process.

The Conjecture module uses knowledge gained from previous iterations to determine what to ask during the next CDEA iteration, should another CDEA iteration be needed. The Design module determines which statistical data structure (experimental design) to use in order to answer the questions from the Conjecture module with available resources. The Experiment module performs the experimental runs (each experimental run is a single implementation of given input parameters) and records the experimental results. The Analyze module performs the appropriate tests on the experimental run results, and outputs the tests' conclusions of the current 


\subsection{Response Surface Methodology}

experimental design. Essentially, the Analysis module uses the data provided by the Experiment module (which performs the experimental runs constructed by the Design module), to compute the answers to statistical questions posed by the Conjecture module. It is this methodology that will be explored and improved.

\subsubsection{Design of Experiments}

An experimental design is a collection and arrangement of experimental runs designed to gain the information most relevant to the project goals with a minimum of resource and time, while compensating for nuisance factors (elements which may affect the response, but are not of interest to the experimenter) (Montgomery (62)). The available resources are analyzed to determine what experimental designs are viable, and which experimental design would be most appropriate for satisfying the goals of the research. The experimental design is chosen according to how and why each factor is selected, and how each is suspected to interact with the other factors of interest.

Even though some projects may be small enough to complete with a single experimental design, scientific investigations usually require more than one set of experiments. Thus, many designs are intended to be part of a larger sequence of experimental designs. Although each experimental design has an internal mathematical justification for its structuring, it is up to the experimenter to properly choose and integrate the designs into an overall project. For more details, see Appendix A.1. 


\subsection{Response Surface Methodology}

\subsubsection{Automating ARC-RSM}

Many researchers perform experimentation without properly justifying the experimental designs. Without adequate justification of choice of the experimental designs, the conclusions of the experimentation cannot be justified. Any automation of resource-constrained response surface methodology must adequately address the following issues:

- The results of current experimentation must affect the kind of experimentation that will occur next.

- The number and nature of the possible results of current experimentation can only be determined after its experimental design has been constructed. If the experimental design is changed, the number and nature of possible results would necessarily change as well.

- The cost of an experimental design affects what can be allocated for future experimentation.

- In order for planning elements to choose and possibly change their own individual experimental designs and evaluation methods, the elements must be able to determine what experiments could follow any experimental design performed, and ensure that the sum of the expenses required to carry out all of the experimentation does not exceed the given budget for time and resources. 


\subsection{Response Surface Methodology}

Another concern is communication and comparison between different research communities, since their philosophical interpretations of response surface methodology can be disparate enough that it is difficult to transfer knowledge from one response surface methodology interpretation to another. In addition, overall resource management is not discussed in the current Response Surface Methodology literature. This is true even with the examples in this section, which are the best available.

Figure 2.2 from Neddermeijer, van Oortmarssen, Piersma, and Dekker (66) depicts a meta level description for automating response surface methodology, except that it assumes that screening has already been performed. 


\subsection{Response Surface Methodology}

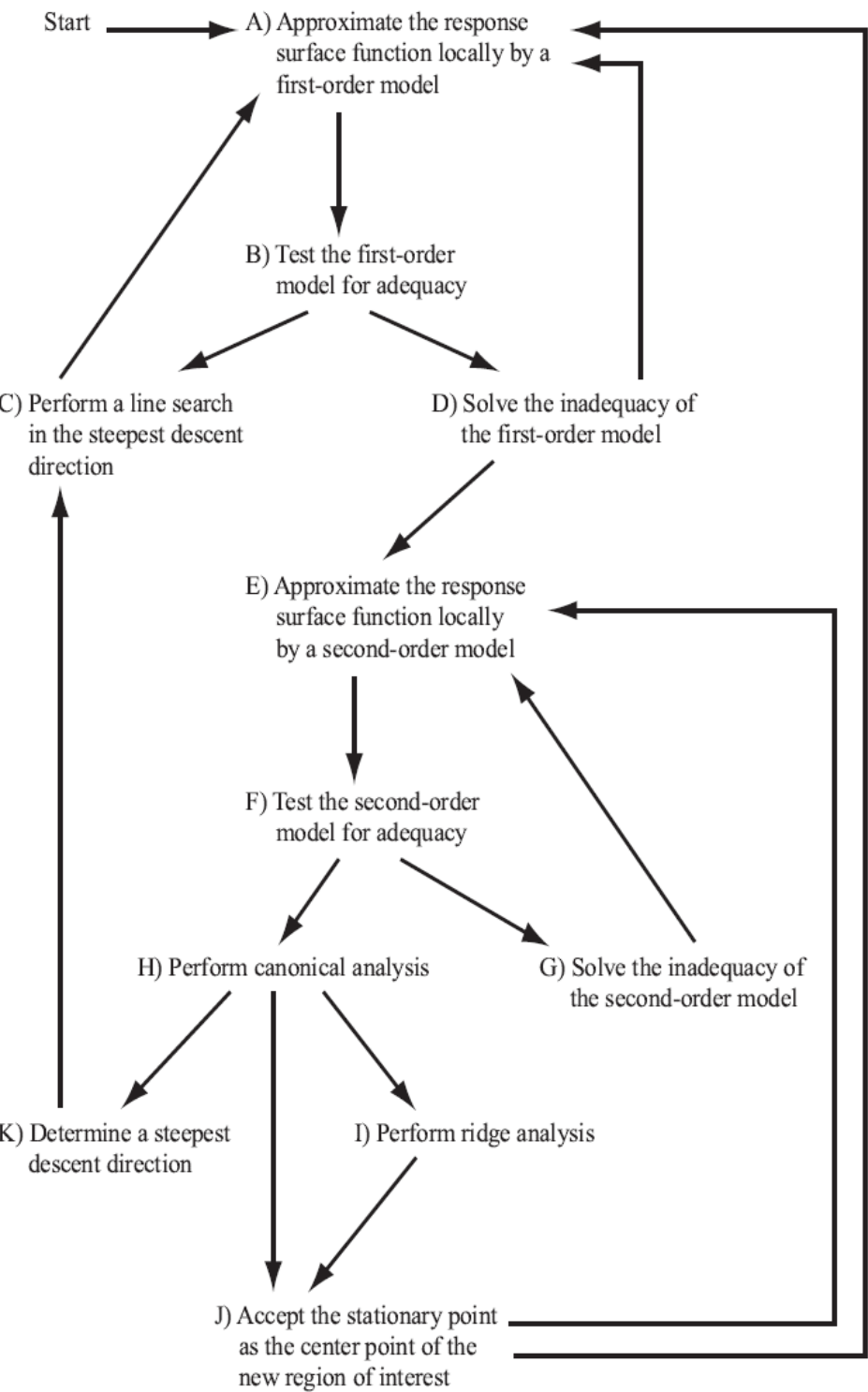

Figure 2.2: Framework for an Automated Response Surface Methodology Algorithm from Neddermeijer, van Oortmarssen, Piersma, and Dekker (66), to show a current attempt to automate response surface methodology. 


\subsection{Response Surface Methodology}

Additionally, their algorithm does not allow for the introduction of new strategies and elements, and its terminating condition is not clear. Schamburg and Brown (80) present a qualitative methodology for approaching response surface methodology from a systems point of view. In another paper from the same year, Brown and Schamburg (15) present a more descriptive approach to response surface methodology, shown in Figure 2.3. However, the framework misses details regarding model selection, dependencies between response surface methodology iterations, and termination within finite time. 


\subsection{Response Surface Methodology}

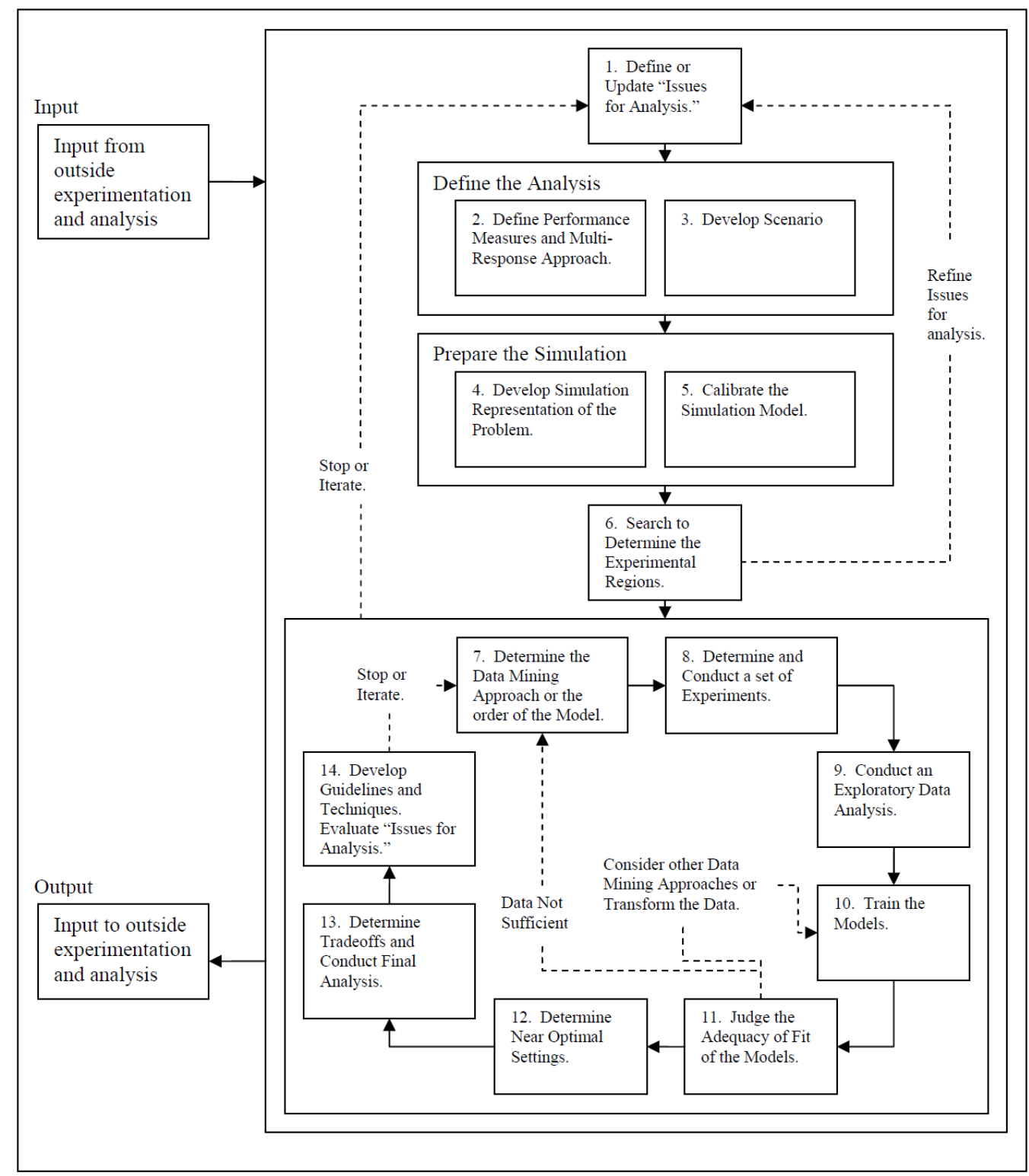

Figure 2.3: Diagram from Brown and Schamburg (15) describing their "Modified Response Surface Methodology", to show a different kind of attempt to automate response surface methodology. 


\subsection{Response Surface Methodology}

The framework of Nicolai and Dekker (68) implements a traditional approach to response surface methodology, and as such, there is no assurance that their algorithm

will terminate. Although their paper describes game theoretic approaches, its descriptions and integrations of response surface methodology methods within the described framework are vague. Screening is also assumed to have been performed before the start of their algorithm, which is shown in Figure 2.4 . 


\subsection{Response Surface Methodology}

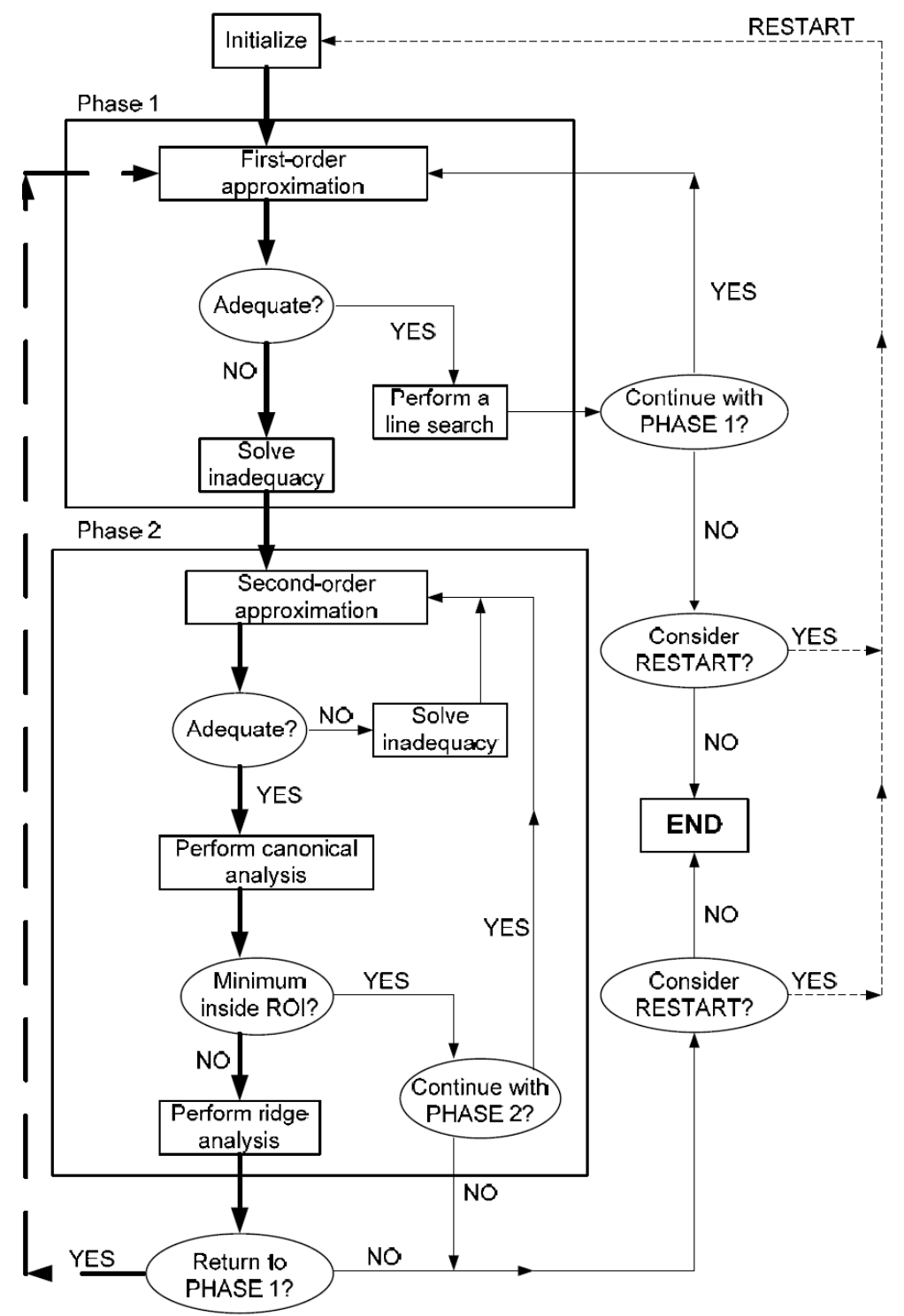

Figure 2.4: Diagram from Nicolai and Dekker (68) describing their response surface methodology framework, to show another previous attempt to automate response surface methodology.

$\mathrm{kl}$ 


\subsection{Systemic Representation}

\subsubsection{Resource-Constrained Project Scheduling}

The resource-constrained project scheduling problem is the problem of arranging different activities required to complete a specific project, while making sure the resources used by those activities are not exhausted before the project is complete. There is no general consensus for solving the problem, but there are examples demonstrating near optimal solutions, given available methods.

In particular, if the experiments take the same amount of time and the cost of each experiment is known beforehand, there are many well known solutions. For projects with interdependent activities, precedence, which states what activities must be completed before others can be attempted, becomes an important issue. An example from Ben Abdelaziz, Krichen, and Dridi (6) is provided in Figure 2.5.

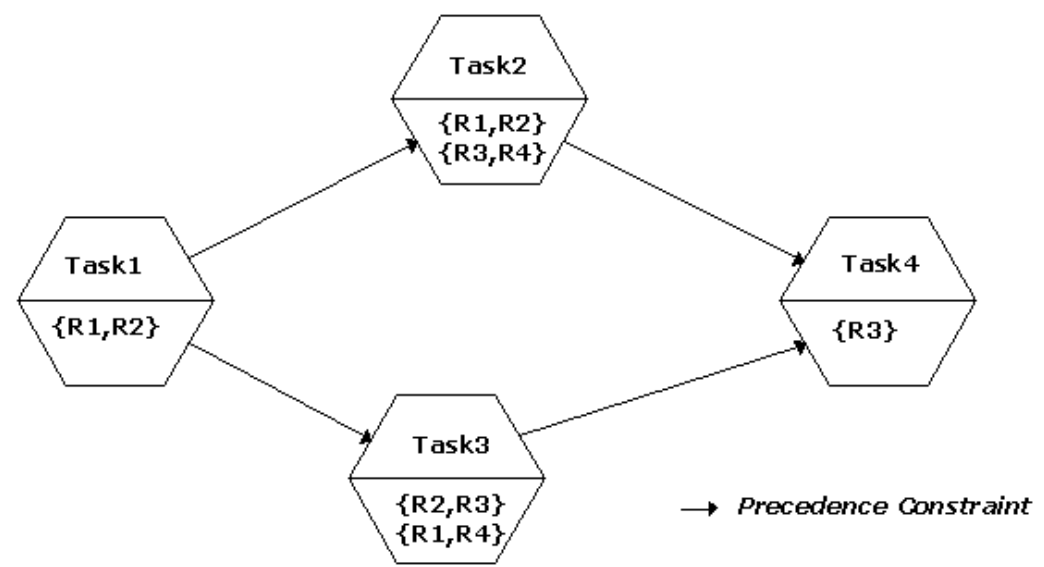

Figure 2.5: Structure of the resource-constrained project scheduling problem, where the resources are labeled as $R_{1}$ through $R_{4}$, from Ben Abdelaziz, Krichen, and Dridi (6) 


\subsection{Systemic Representation}

From this common structure, there are several ways to approach the resourceconstrained project scheduling problem. Fleszar and Hindi (30) and Ben Abdelaziz, Krichen, and Dridi (6) introduce a partial order, but most use metaheuristics to guide behavior (Fleszar and Hindi (30), Kolisch and Hartmann (51) (52) (53), Ouelhadj and Petrovic (74)). However, in response surface methodology, the sequence of experimental designs is not fully known, since each experimental design is a reaction to the analysis of the last experimental design. Consequently, existing resource-constrained project scheduling approaches may be useful for an initial project design, but an implementation that properly accounts for this lack of knowledge requires additional mathematical structures that the current resource-constrained project scheduling approaches cannot or do not incorporate.

\subsubsection{Game Theory/Decision Theory}

Game theory is based around analyzing games, where a game is a system in which there are multiple interdependent elements(players) that each follow formal rules(Osborne and Rubinstein (73)). Each player forms a strategy out of available choices to pursue a goal while considering the other players' goals and available choices. By analyzing the possible strategies each player can have, as well as the possible outcomes of each player following a particular available strategy, it is possible to study the interrelationships of the players in the system. Decision theory can be considered to be part of game theory by considering the decision process to be a game against nature, where nature is considered to be a player that does not care 


\subsection{Systemic Representation}

about the decision maker's actions (Parsons and Wooldridge (75)).

Game theory, however, is generally probabalistic rather than statistical, whereas this research focuses on situations where the probabilities are unknown. Like game theory, statistics does not put the focus on what the result will be, but whether or not the conclusion will meet certain standards of correctness. These standards can be mathematical, statistical, or correctness of information known about the system being studied.

An analytical task produces a result from a set of multiple possible results, based on observations from a system which is not totally understood, and so there is incomplete and imperfect information about the results of each choice. Therefore, in a game where analytical tasks make up the choices, that game must be considered as having incomplete and imperfect information.

The following example shows the process of attempting to reduce the variety of choices to a single choice, in order to determine a decision. To begin, Figure 2.6 shows an example of a decision maker's possible choices $\left(\theta_{1}-\theta_{4}\right)$, taken from a set of generally applicable actions: 


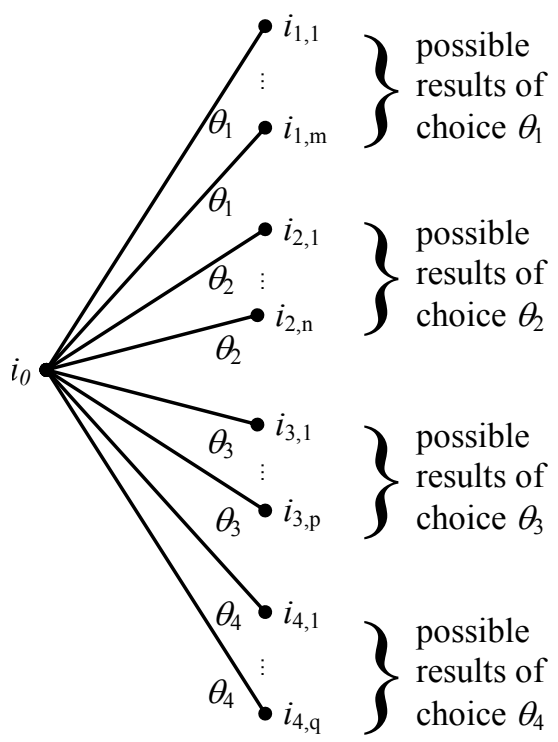

Figure 2.6: Initial collection of considered choices a decision maker has available.

First, since resources are limited, the choice(s) that cannot be afforded must be removed. In this case, assume that the cost of $\theta_{4}$ exceeds the budget for the project and therefore must be removed. 


\subsection{Systemic Representation}

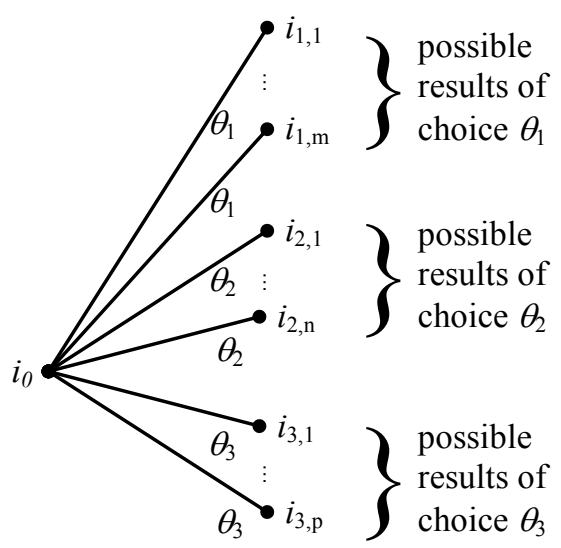

Figure 2.7: Current choices a decision maker has considered available, after unaffordable choices are removed.

Next, the choice(s) that cannot produce results of interest to the sponsor are removed. In this example, perhaps $\theta_{1}$ includes an expensive test for something the sponsor does not consider relevant to the given problem.

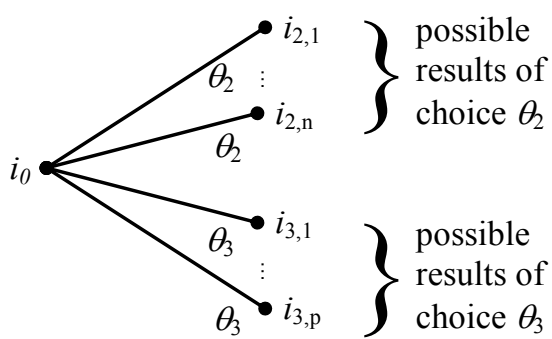

Figure 2.8: Current choices a decision maker has available, after choices with uninteresting results are removed.

Then, the choice(s) that do not incorporate the desired standards of correctness are removed. For example, $\theta_{3}$ might produce a relevant result, but may not sufficently address the kinds of error that the sponsor is most concerned with. 


\subsection{Systemic Representation}

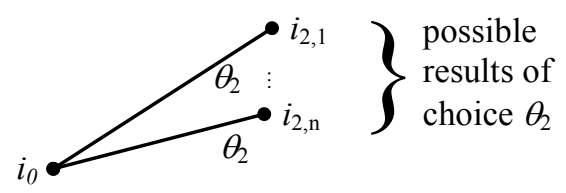

Figure 2.9: Remaining choices a decision maker has available, after choices that do not incorporate the desired standards of correctness are removed.

Since exactly one choice remains, then that is the preferred choice. If there are no choices left, then either the circumstances of the game (amount of available resources, nature of the goal, etc.) or the variety-reducing criteria need to be changed to allow at least one choice. If there are multiple choices left, then the variety-reducing criteria needs to be adjusted or expanded to differentiate between the choices, making sure to provide justification for the nature of the changes. 
3

\section{Introduction to Adaptive}

\section{Resource-Constrained Response}

\section{Surface Methodology (ARC-RSM)}

\subsection{Generating approaches to RSM from collec- tions of experimental designs}

Design of experiments works locally, based on current knowledge, which means that the most basic research strategy is a collection of experimental designs. An experimental design can have the order of its runs randomized, but the runs themselves are predetermined before any run of the experimental design is performed. Following this, research strategies need to be fixed as much as possible, since there is no point in 


\subsection{Response Surface Methodology Iterations in Terms of Collective}

Behavior

trying to 'trick' nature, to avoid confusing randomness within the research strategy for randomness within the system being observed.

Termination conditions are not well described in RSM literature. Consequently, this methodology introduces its own means for incorporating termination conditions. Also, boundary conditions need to be incorporated in order to insure both termination of the research strategy design process, and the termination of the research strategy in application. Since RSM is an iterative knowledge discovery process, the termination and boundary conditions will be based around the emerging behavior of research strategies. For example, resource needs may not be entirely determinable from the initial state, so feedback will be used to consider potential future needs that may not be anticipated with one experimental design.

\subsection{Response Surface Methodology Iterations in Terms of Collective Behavior}

First, the interpretation of response surface methodology used in this research will be clarified. As described in Section 2.1.1, each iteration of response surface methodology is made up of four interacting elements; Conjecture (C) Design (D), Experiment (E) and Analyze (A), which are shown in Figure 3.1. 


\subsection{Response Surface Methodology Iterations in Terms of Collective}

Behavior

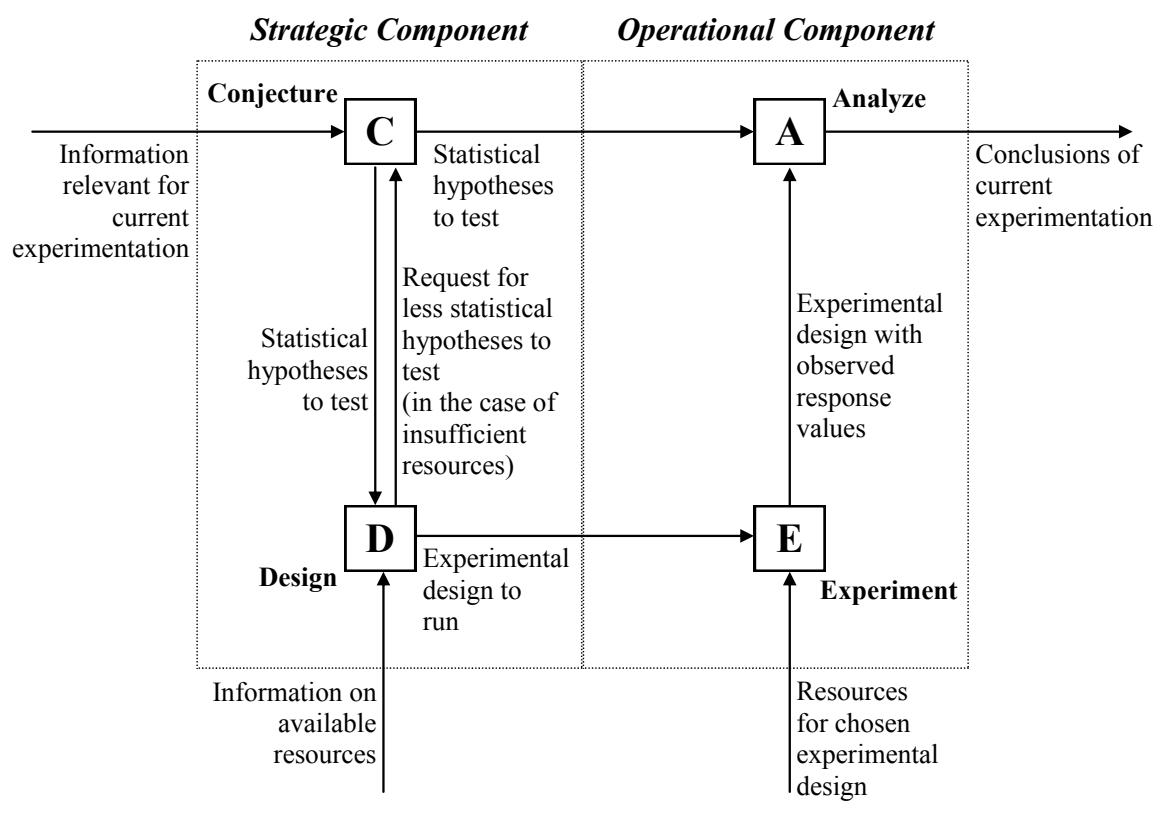

Figure 3.1: Visual description of a single CDEA iteration of response surface methodology.

Each CDEA element (Conjecture, Design, Experiment, Analyze) has its own internal processes and communicates information with other elements as described in Figure 3.1, working together on different parts of a single experimental design. Therefore, each element must have a response for whatever information it receives, and its output must be valid input for its recipient. The behaviors of $\mathrm{E}$ and $\mathrm{A}$ are mechanical responses to $\mathrm{C}$ and $\mathrm{D}$, and form the operational component. The $\mathrm{C}$ and $\mathrm{D}$ elements determine what experimental design and associated analytical tasks can and should be run, and comprise the strategic component. C and D must be able to work with each other, and must operate in compatibility with $\mathrm{E}$ and $\mathrm{A}$.

In terms of individual CDEA iterations, each iteration is the response to the 


\subsection{Research Strategy Requirements}

real world results of previous experimentations. Therefore, each iteration can be considered a "move" in a game against nature. Since the strategic component of a CDEA iteration is where planning occurs, the output of the strategic component of a CDEA iteration will be called a planned move. A collection of planned moves will be called a research strategy.

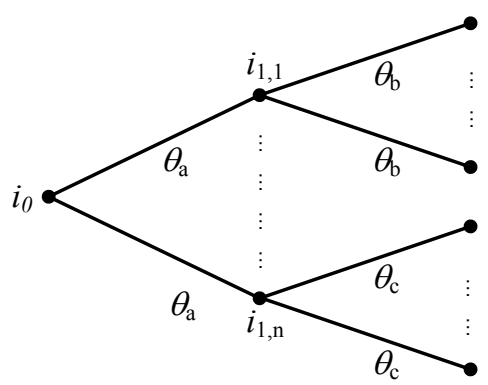

Figure 3.2: Visual description of a research strategy.

The next section describes the requirements that a research strategy must meet.

\subsection{Research Strategy Requirements}

A scientific investigation involving the collecting of empirical data is not usually completed with a single experimental design, but rather with a sequence of experimental designs whose nature develops according to the results of evolving knowledge. Consequently, an overall methodology coherently linking the individual CDEA iterations is required, and such a methodology does not currently exist.

Regardless of the situation, a researcher following a research strategy should be able to statistically derive the sponsor-desired information within the resource con- 


\subsection{Research Strategy Requirements}

straints. Each experimental design uses a predetermined and finite amount of resource, and produces results from a known set of possible conclusions that can be reduced algebraically to a known finite set of significant conclusions. Furthermore, each subsequent experimental design is a reaction to the conclusions made by that previous experimental design. For example, the first experimental design might have a single hypothesis test, so the next experimental design invoked would need to develop a reaction according to the null hypothesis being rejected, and be able to react to the null hypothesis not being rejected. In general, a research strategy should be a dynamic plan that is defendable, efficient, terminating in finite time, and within budget. It should be noted that an effective research strategy might not produce the desired result. For instance, it may be that no controllable factor considered by the sponsor has any significant effect on the response $y$, so that would be the honest conclusion, even if it fails to achieve the intended overall goal of the project's sponsor.

There are three types of knowledge that are considered when implementing a CDEA iteration; facts, goals, and resources. Facts describe the variables under study and what has been determined about them. Goals describe what knowledge the sponsor wants to obtain. Resources describe the current state of testing conditions. In more detail:

- Facts

A fact predicate, or fact for short, is a predicate that represents a statement about at least one system element determined to be relevant directly or indirectly by the designer and/or sponsor ( i.e. $\operatorname{scrPass}\left(x_{1}, y\right)$, meaning " $x_{1}$ has passed the 


\subsection{Research Strategy Requirements}

screening test with respect to $\left.y^{\prime \prime}\right)$.

Examples:

- conclusions of experimental designs determined by designer and/or sponsor

- initial sponsor-defined assumptions

- Goals

A goal command, or goal for short, is a command that represents a client-specified objective ( i.e. opt $\left(x_{1}, y\right)$, meaning "optimize the response $y$ in terms of the predictor $\left.x_{1}^{\prime \prime}\right)$.

Examples:

- what the sponsor wants to know

- Resources

A resource predicate is a predicate that states information about the available resources ( for example, avail $(3, l, t)$, meaning "only 3 samples of $l$ for each unit of time $\left.t^{\prime \prime}\right)$.

Examples:

- amount of time left available for experimentation

- types of physical resource available

- available amounts of each type of resource 


\subsection{Research Strategy Requirements}

- constraints on the accessibility of each type of resource

Therefore, let $\mathscr{F}$ be the set of all finite sets of facts that a team could consider, $\mathscr{G}$ be the set of all finite sets of goals a team could be given, and $\mathscr{H}$ be the set of all finite sets of possible resource states (finite quantities and types of resource). Let $I=\mathscr{F} \times \mathscr{G} \times \mathscr{H}$, which shall be referred to as the knowledge state index set, and an element of $I$ will be considered a knowledge state index. The knowledge state index representing the state of knowledge at the beginning of the research strategy(the knowledge given by the sponsor) is called the initial knowledge state index. Since this research works with the knowledge state index set $I$, it is useful to have clear, technical language for interacting with $I$.

Definition 3.1. For any $i=(F, G, H) \in I$, define $i_{\mathscr{F}}:=F, i_{\mathscr{G}}:=G, i_{\mathscr{H}}:=H$. For any $J \subseteq I$, define

$$
\begin{aligned}
& J_{\mathscr{F}}:=\{F \in \mathscr{F}:(F, G, H) \in J \text { for some } G \in \mathscr{G}, H \in \mathscr{H}\} \\
& J_{\mathscr{G}}:=\{G \in \mathscr{G}:(F, G, H) \in J \text { for some } F \in \mathscr{F}, H \in \mathscr{H}\} \\
& J_{\mathscr{H}}:=\{H \in \mathscr{H}:(F, G, H) \in J \text { for some } F \in \mathscr{F}, G \in \mathscr{G}\}
\end{aligned}
$$

The research strategy must have a planned move for the initial knowledge state index, and a planned move for each knowledge state index that could follow the initial knowledge state index until experimentation is complete. 


\subsection{Preference Function Lists}

Note that a research strategy is designed to specify the implementation and analysis of real-world experimental runs, but experimental runs are not performed during its strategy development process.

\subsection{Preference Function Lists}

There are several important things to consider regarding the response surface methodology procedure:

1. Each experimental design is determined by the conclusions that exist at the beginning of the CDEA iteration and the overall goal. Also, due to resource constraints, there is always a finite upper bound for how many experiments can be performed.

2. The only operation that can be considered expensive is the actual running of experiments, expressed in terms of time and resources.

3. The information available at any point must be considered imperfect and incomplete, since the environment is at least partially unknown, and there is a possibility of an incorrect conclusion at every stage of research.

A preference function list is a list of preference-generating functions (which have been created by the sponsor and experimental designer), including local decision preferences and success conditions, in order to determine a choice where several choices are technically acceptable. The designer transmits information to the machine through the preference function list created by the designer with the sponsor's information 


\subsection{Preference Function Lists}

and approval (agreement between designer and sponsor on what the sponsor really wants). Each preference function represents the sponsor's response to a particular question that an experimental designer needs to ask the sponsor.

Definition 3.2. A preference function is a function $\operatorname{pref}_{k}^{p}: I \rightarrow A$ where $I$ is the knowledge state index set and $A$ is a set of preference choices based upon the purpose of the preference function. A sponsor-designer preference function list is a list of preference functions, $\left\{\operatorname{pref}_{k}^{p}\right\}_{k}$, which share a domain I. Let $P$ be the set of all sponsor-designer preference function lists. Each type of decision made with a sponsordesigner preference function list corresponds directly with a specific function within each of the sponsor-designer preference function lists (for example, determining if the goals are satisfied).

This next set of preference functions are the preference functions that all sponsordesigner preference function lists are required to have. First is the preference function needed to determine successful termination of experimentation.

Definition 3.3. The boolean preference function pref $_{\text {projSat }}^{p}$ within a sponsor-designer preference function list $p \in P$ that determines whether experimentation should be considered successfully finished is called the project satisfaction function of $p$.

Next is the preference function that determines how important it is that experimentation can be completed from a given knowledge state index.

Definition 3.4. The nonnegative real-valued preference function pref $_{\text {weight }}^{p}$ within a sponsor-designer preference function list $p \in P$ that determines how important it is 


\subsection{Preference Function Lists}

that experimentation can be completed from a given knowledge state index is called the index priority weight function of $p$.

Then, the preference function that determines if a planned move is acceptable.

Definition 3.5. The nonnegative real-valued preference function $\operatorname{pref}_{\text {thresh }}^{p}$ within a sponsor-designer preference function list $p \in P$ that determines the minimum level of successful feedback (from 0 to 1, 0 being feedback of no success, 1 being feedback of complete success) required for acceptance is called the feedback compromise threshold function of $p$.

Figure 3.3 presents a visual demonstration of the overall method in terms of the Ashby goal-oriented regulator described in Section 1.4 .

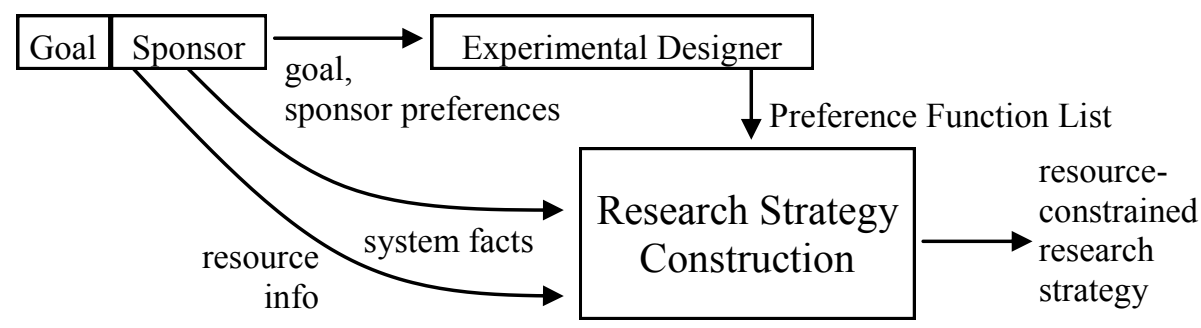

Figure 3.3: Black-box diagram of the generation of a research strategy.

Based on these ideas, this research constructs a formal language for the decisions made in response surface methodology that can determine if a research strategy can be constructed from sponsor-given information, according to sponsor-defined conditions. 


\subsection{General Algorithm for Constructing Research Strategies}

\subsection{General Algorithm for Constructing Research Strategies}

Suppose that we had an initial knowledge state index $i_{0}$ as defined in Section 3.3 . and a sponsor-designer preference function list $p$ as defined in Section 3.4 .

First, $p$ is used to create a task list $T$ to be performed at $i_{0}$, and an experimental design list $D_{T}$ from which to choose an experimental design that $T$ will be performed on, such that the expense of performing $T$ is not greater than the available resources. Figure 3.4 shows a visual examplet.

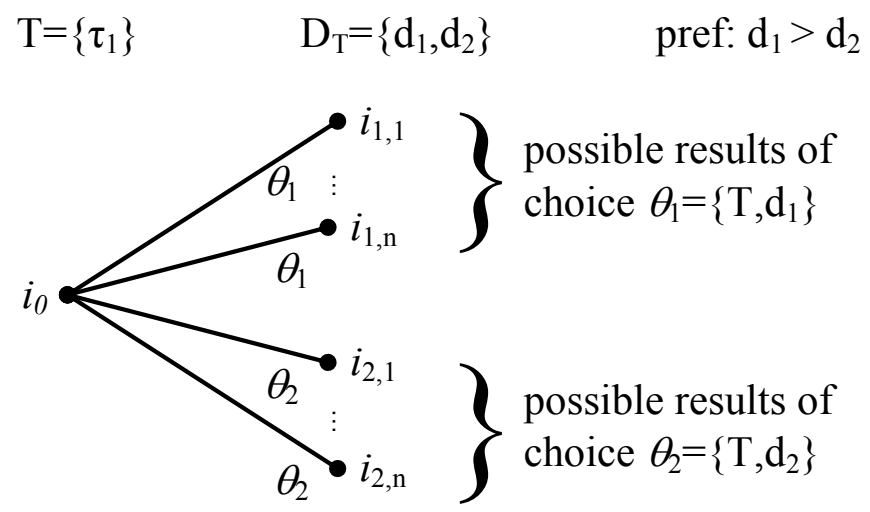

Figure 3.4: Visual example of acceptable choices.

In this example, the choice $\theta_{1}=\left(T, d_{1}\right)$, where $d_{1}$ is the most preferred design in $D_{T}$, will be temporarily considered the initial planned move. Next, this process is continued from the knowledge state indexes after $\theta_{1}$ at $i_{0}\left(i_{1,1}, \ldots, i_{1, n}\right)$ to determine if the project can be completed after the current initial choice. If not, then the choice

\footnotetext{
${ }^{1}$ For the sake of simplicity, there is only one analytical task and two experimental designs.
} 


\subsection{General Algorithm for Constructing Research Strategies}

$\theta_{1}=\left(T, d_{1}\right)$ is no longer considered, $d_{1}$ is removed from $D_{T}$, and the next most preferred design $d_{2} \in D_{T}$ is selected, and the process begins again for the new choice

$\theta_{2}=\left(T, d_{2}\right)$. Note that $d_{1}$ and $d_{2}$ have the same number of possible results because they have the same task list.

If $D_{T}$ is exhausted without finding a research strategy, then $T$ is reduced by removing the analytical tasks of lowest priority, a new $D_{T}$ is constructed, and the process starts again. The first choice that is completable within the resource constraints is considered the preferred choice; thus, no other choice is of interest past this point.

If there are no choices left, then either the project circumstances (amount of available resources, nature of the goal, etc.) or the variety-reducing criteria need to be changed to allow at least one choice. 
4

Creation of research strategies in ARC-RSM

\subsection{ARC-RSM Algorithm Overview}

In order to organize the research process, the basic concepts of RSM are listed in order of importance, greatest to least:

1. affordability of chosen research strategy (without this condition being met, the research strategy can't be done)

2. meeting of satisfaction conditions (without this condition being met, the research strategy may not be useful)

3. performing of priority analytical tasks (experimental designs exist in order to 


\subsection{ARC-RSM Algorithm Overview}

have analytical tasks performed on them)

4. selection of preferred experimental designs

Figure 4.1 is a visual representation of the choice selection process that will be described in this section: 


\subsection{ARC-RSM Algorithm Overview}

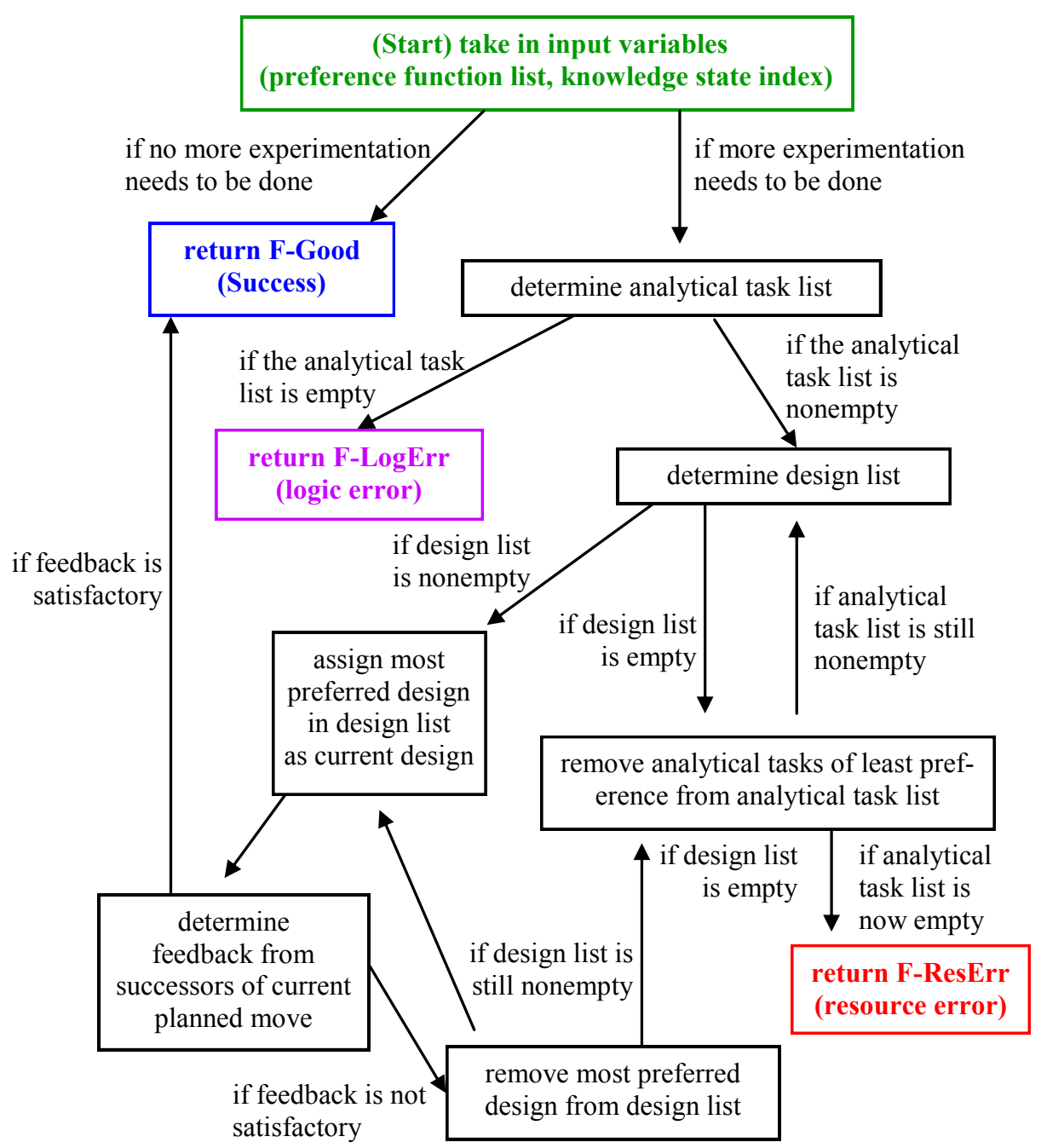

Figure 4.1: Visual representation of the algorithm for the construction of a planned move. 


\subsection{Basic ARC-RSM Analytical Task Terms}

\subsection{Basic ARC-RSM Analytical Task Terms}

The following definition describes analytical tasks as they will be interpreted during the construction of a research strategy.

Definition 4.1. An analytical task $\tau$ is a function which represents a particular statistical process (for example, screening a predictor), directed towards an analytical target $x$ (for example, a predictor being screened). A potential argument for an analytical task is represented as an ordered pair $\left(x, a_{\tau \mid i}\right)$, where $x$ is the analytical target, and $a_{\tau \mid i}$ is the additional information needed to specify the analytical task at

$i \in I$ (levels of significance, etc.), and returns the set of potential facts representing the possible conclusions of that process (for example: \{the predictor passes screening, the predictor fails screening\}). Let $T$ be the set of all analytical tasks, $X$ be the set of all analytical targets, $A_{\tau}$ be the set of possible arguments for a given analytical task $\tau \in T$, and $A_{T}=\bigcup_{\tau \in T} A_{\tau}$.

This next set of domains are needed to clarify where analytical tasks can be used, and to specify the analytical tasks according to sponsor-designer requirements.

Definition 4.2. For a given $\tau \in T$, the preference function targetList ${ }_{\tau}^{p}$ takes in a knowledge state index $i \in I$, and returns the list of analytical targets that $\tau$ is to be used on at $i$. target List $t_{\tau}^{p}(i)$ is the target list of $\tau$ at $i$ given $p$.

Definition 4.3. For a given $\tau \in T$, define $I_{\tau} \subseteq I$ as the set of knowledge state indexes where $\tau$ is applicable (contains analytical targets for $\tau$, etc.). $I_{\tau}$ is the knowledge criteron of $\tau$. For $K \subseteq T, I_{K}:=\bigcap_{\tau \in K} I_{\tau}$ is the knowledge criteron of $K$. 


\subsection{Basic ARC-RSM Analytical Task Terms}

The analytical task specification function is the preference function which specifies and prioritizes each instance of an analytical task in a task list.

Definition 4.4. For a given $\tau \in T$, the preference function $\operatorname{pref}_{\tau}^{p}$ within a sponsordesigner preference function list $p \in P$ that is used to determine user arguments and priorities for an analytical task $\tau \in T$ is called the analytical task specification function of $p$ for $\tau$. Specifically, for any $i \in I$,

$$
\begin{aligned}
& \operatorname{pref}_{\tau}^{p}(i):=\left\{\tau_{x \mid i}^{p}=(\tau,(x, a), k): x \in \operatorname{target} L i s t_{\tau}^{p}(i), a \in A_{\tau}, k \in[0,1]\right\} \\
& \text { For } t=(\tau,(x, a), k) \in \operatorname{pref}_{\tau}^{p}(i) \text { define } t_{\text {task }}:=\tau, t_{\text {arg }}:=(x, a), \text { and } \\
& t_{\text {priority }}:=k .
\end{aligned}
$$

This next notation represents the range of a specified analytical task.

Definition 4.5. For $S \subseteq \operatorname{pref}_{\tau}^{p}(i)$, define

$$
\vec{S}:=\{\tau(x, a):(\tau,(x, a), k) \in S \text { for some } k \in[0,1]\}
$$




\subsection{Basic ARC-RSM Experimental Design Structures}

\subsection{Basic ARC-RSM Experimental Design Struc- tures}

Since changing an experimental design can change the amount of resources it requires, each experimental design will be considered fully specified.

Definition 4.6. Let $D$ be the set of all unperformed experimental designs to be considered. Each element in $D$ represents a specific experimental design setup.

Since experimental designs will be chosen with respect to a previously constructed task list and real resource constraints, these next definitions represent the domains within which an individual experimental design can be chosen.

Definition 4.7. For a given $d \in D$, define $I_{d} \subseteq I$ as the set of knowledge state indexes where $d$ can be performed affordably. $I_{d}$ is the affordability criteron of $d$.

Definition 4.8. For a given $K \subseteq T$ and $U \subseteq X$, define $D_{K \mid U} \subseteq D$ as the set of experimental designs that can incorporate $U$, and that $K$ can be performed upon. $D_{K \mid U}$ is the application criteron of $K$ given $U$, and $D_{K}:=\left\{d: d \in D_{K \mid U}\right.$ for some $U \subseteq$ $X\}$ is the application criteron of $K$.

Definition 4.9. For a given $K \subseteq T$ and $d \in D_{K}$, define $I_{d \mid K}:=I_{d} \cap I_{K} . I_{d \mid K}$ is the strategic criteron of d given $K$.

Since exactly one experimental design will be used for each task list, the preference ordering of experimental designs will be strict. 


\subsection{Basic ARC-RSM Experimental Design Structures}

Definition 4.10. For a given $K \subseteq T$ and $d \in D_{K}$, the preference function $\operatorname{pref}_{d \mid K}^{p}$ : $I_{d \mid K} \rightarrow[0,1]$ within a sponsor-designer preference function list $p \in P$ that is used to determine the user preference for the experimental design d ( 0 is no interest, 1 is highest interest) is called the experimental design preference ordering function of $p$ for $d$ given $K$. It should be noted that for any $i \in I$ and distinct $d_{1}, d_{2} \in D_{K}$, that $\operatorname{pref}_{d_{1} \mid K}^{p}(i) \neq \operatorname{pref}_{d_{2} \mid K}^{p}(i)$ if either $\operatorname{pref}_{d_{1} \mid K}^{p}(i)$ or pref $_{d_{2} \mid K}^{p}(i)$ are nonzero, in order to maintain strict preferences between experimental designs.

Definition 4.11. For $p \in P$, define

$$
D_{p}:=\left\{d: d \in D, \operatorname{pref}_{d \mid K}^{p}(i)>0 \text { for some } K \subseteq T \text { and } i \in I\right\}
$$

$D_{p}$ is the preferred design set given $p$.

It should be noted that, as a rule of thumb, the priority/preference of any given task/design should be zero, unless explicitly defined by the client and designer. Even if there is no strict difference in client/designer preference between two or more designs, the experimental design preference ordering function must be specified as if there is, even if it is by constructing research strategies for all preference ordering permutations for choices of equal client/designer preference.

Resource cost is a vital part of this research. Consequently, a formal language is needed to represent the counting arguments required to keep track of resource cost as resource allocation is planned out.

Definition 4.12. For $i \in I$ and $d \in D$, define cost $(d)$ as the resource cost of using 


\subsection{Ordering of Analytical Tasks}

$d$. Let $i_{\mathscr{H}}-\operatorname{cost}(d)$ represent the change of resources from $i_{\mathscr{H}}$ after $d$ is performed, and let $i_{\mathscr{H}} / \operatorname{cost}(d)$ equal the maximum number of times that cost $(d)$ could be removed from $i_{\mathscr{H}}$. For $d_{1}, d_{2} \in D, \operatorname{cost}\left(d_{1}\right) \leq \operatorname{cost}\left(d_{2}\right)$ means that $d_{2}$ has at least the same change in resources as $d_{1}$. For $d \in D$ and $i \in I$, cost $(d) \leq i_{\mathscr{H}}$ means that $d$ is affordable at $i$.

\subsection{Ordering of Analytical Tasks}

This section describes how the lists of analytical tasks are collected, reduced, specified, sorted and accessed.

The first list is for what analytical tasks are available at a given knowledge state index.

Definition 4.13. For $i \in I$, define

$$
T_{i}:=\left\{\tau: \tau \in T, i \in I_{\tau}\right\}
$$

$T_{i}$ is called the available analytical task list in terms of $i$.

The next list is for the analytical tasks that are available at a given knowledge state index and are of interest according to a given sponsor-designer preference function list. 


\subsection{Ordering of Experimental Designs}

Definition 4.14. For $i \in I$ and $p \in P$, define

$$
T_{(i, p)}:=\left\{t: t \in \operatorname{pref}_{\tau}^{p}(i), \tau \in T, i \in I_{\tau}, t_{\text {priority }}>0\right\}
$$

$T_{(i, p)}$ is called the specified analytical task list in terms of $(i, p)$.

This next list is to determine what analytical tasks are to be removed from a given analytical task list, according to a given sponsor-designer preference function list, if feedback indicates they cannot all be performed.

Definition 4.15. For $i \in I, p \in P$, and $S \subseteq T_{(i, p)}$, define

$$
\operatorname{lpt}(i, p):=\left\{t: t \in S, t_{\text {priority }} \leq \tau_{\text {priority }} \text { for all } \tau \in S\right\}
$$

$\operatorname{lpt}(i, S, p)$ is called the lowest priority analytical task list by $p$ in $S$ at $i$.

\subsection{Ordering of Experimental Designs}

This section describes how the lists of experimental designs are collected, reduced, specified, sorted and accessed.

The first list is for what experimental designs are available at a given knowledge state index for a given analytical task list.

Definition 4.16. For $i \in I$ and $K \subseteq T$, define

$$
D_{(i, K)}:=\left\{d: d \in D, i \in I_{d \mid K}\right\}
$$




\subsection{Ordering of Experimental Designs}

$D_{(i, K)}$ is called the available design list in terms of $(i, K) . D_{i}:=D_{\left(i, T_{i}\right)}$ is called the available design list in terms of $i$.

The next list contains the experimental designs that are available at a given knowledge state index for a given analytical task list, and are of interest according to a given sponsor-designer preference function list.

Definition 4.17. For $i \in I, K \subseteq T$, and $p \in P$ define

$$
D_{(i, K, p)}:=\left\{d: d \in D_{(i, K)}, \operatorname{pref}_{d \mid K}^{p}(i)>0\right\}
$$

$D_{(i, K, p)}$ is called the specified design list in terms of $(i, K, p) . D_{(i, p)}:=D_{\left(i, T_{(i, p)}, p\right)}$ is called the specified design list in terms of $(i, p)$.

Since only one experimental design can be chosen, the next function determines how to choose the most preferred experimental design from a list of experimental designs

Definition 4.18. For $i \in I, K \subseteq T$, nonempty $L \subseteq D$ and $p \in P$, define

$$
\operatorname{mpd}(i, K, L, p):=m
$$

where $m \in L$ and $\operatorname{pref}_{m \mid K}^{p}(i) \geq \operatorname{pref}_{d \mid K}^{p}(i)$ for all $d \in L$, and $m p d(i, K, \emptyset, p):=\emptyset$. $\operatorname{mpd}(i, K, L, p)$ is called the most preferred design by $p$ in $L$ at $i$ for $K$. 


\subsection{Basic Requirements for ARC-RSM Structures}

\subsection{Basic Requirements for ARC-RSM Structures}

This section includes special requirements that must be met in order to satisfy later theorems.

First, for each goal and sponsor-designer preference function list, there must be some set of conditions that will satisfy the goal according to the sponsor-designer preference function list.

Definition 4.19. Let $p \in P$. If, for any $G \in \mathscr{G}$, there exists an $i \in I$ such that $i_{\mathscr{G}}=G$ and $\operatorname{pref}_{\text {projSat }}^{p}(i)=$ True, then $p$ is satisfiable with respect to $(I, T, D)$.

If each $p \in P$ is satisfiable with respect to $(I, T, D)$, then $P$ is satisfiable with respect to $(I, T, D)$.

Next, conclusions must be distinct from each other to prevent logical contradictions.

Definition 4.20. For a given $i \in I$, define $\left(i_{\mathscr{F}}\right)^{*} \subseteq \bigcup_{F \in \mathscr{F}} F$ as the set of facts containing $i_{\mathscr{F}}$ and the facts that contradict any of the facts in $i_{\mathscr{F}}$ (for example, if $x_{1}$ could be blue or red, and $i_{\mathscr{F}}$ contained the fact that $x_{1}$ was blue, then $\left(i_{\mathscr{F}}\right)^{*}$ would contain the fact that $x_{1}$ was blue, and the fact that $x_{1}$ is red). ( $\left.i_{\mathscr{F}}\right)^{*}$ is called the logical span of $i_{\mathscr{F}}$ in $\mathscr{F}$.

Definition 4.21. For a given $\tau \in T$, if $\tau\left(x, a_{\tau \mid i}\right) \cap\left(i_{\mathscr{F}}\right)^{*}=\emptyset$ for any $i \in I_{\tau}$ and $\left(x, a_{\tau \mid i}\right) \in \operatorname{dom} \tau$, then $\tau$ is a valid analytical task.

If each $\tau \in T$ is a valid analytical task, and $\left(F_{1}\right)^{*} \cap\left(F_{2}\right)^{*}=\emptyset$ for $F_{1} \in \operatorname{ran} \tau_{1}$ and 


\subsection{Basic Requirements for ARC-RSM Structures}

$F_{2} \in \operatorname{ran} \tau_{2}$ where $\tau_{1}, \tau_{2} \in T$ such that $\tau_{1} \neq \tau_{2}$, then $T$ is a valid analytical task set.

Next, there must be boundary conditions to ensure both the creation of a research strategy and the implementation of that research strategy are completed in finite time.

Definition 4.22. If a $p \in P$ meets the following requirements:

1. For $i \in I,\left|T_{(i, p)}\right|<\infty$ (finite number of tasks chosen at each stage)

2. For $i \in I, \mid$ ran $\tau_{p} \mid<\infty$ for each $\tau_{p} \in T_{(i, p)}$. (Each task has a finite number of possible conclusions)

3. For $i \in I,\left|D_{(i, K, p)}\right|<\infty$ for each $K \subseteq T$. (finite number of experimental designs considered for each set of analytical tasks)

4. For any $d_{1} \in D_{p}$, there exists a $d_{2} \in D_{p}$ such that $0<\operatorname{cost}\left(d_{2}\right) \leq \operatorname{cost}\left(d_{1}\right)$, and there does not exist a $d_{3} \in D_{p}$ such that $\operatorname{cost}\left(d_{3}\right)<\operatorname{cost}\left(d_{2}\right)$. (minimum cost boundaries for experimentation)

then $p$ is bounded with respect to $(I, T, D)$.

If each $p \in P$ is bounded with respect to $(I, T, D)$, then $P$ is bounded with respect to $(I, T, D)$.

For ARC-RSM, it is required that $T$ be a valid analytical task set, and $P$ be satisfiable and bounded with respect to $(I, T, D)$. Therefore, these conditions will be assumed in the following sections. 


\subsection{Research Strategy Design Processes}

\subsection{Research Strategy Design Processes}

This next section describes the main processes and states that make up the choice selection process.

The first definition is a very important one: the selection and organization of analytical tasks and experimental designs for a given knowledge state index, according to a given sponsor-designer preference function list.

Definition 4.23. Let $i \in I$ and $p \in P$. Let $\Upsilon_{0}^{(i, p)}=T_{(i, p)}$. Given $\Upsilon_{k}^{(i, p)} \neq \emptyset$ and $D_{\left(i, \Upsilon_{k}^{(i, p)}, p\right)} \neq \emptyset$, let $n_{k}=\left|D_{\left(i, \Upsilon_{k}^{(i, p)}, p\right)}\right|$ and $\Phi_{k}^{(i, p)}=\left\{d_{(j, k)}^{(i, p)}\right\}_{j=1}^{n_{k}}=D_{\left(i, \Upsilon_{k}^{(i, p)}, p\right)}$, where $d_{(1, k)}^{(i, p)}=\operatorname{mpd}\left(i, D_{\left(i, \Upsilon_{k}^{(i, p)}, p\right)}, p\right), d_{(2, k)}^{(i, p)}=\operatorname{mpd}\left(i, D_{\left(i, \Upsilon_{k}^{(i, p)} \backslash\left\{d_{(1, k)}^{(i, p)}\right\}, p\right)}, p\right), \ldots$, and $\Upsilon_{k+1}^{(i, p)}=$ $\Upsilon_{k}^{(i, p)} \backslash \operatorname{lpt}\left(i, \Upsilon_{k}^{(i, p)}, p\right)$. Else, then let $n_{k}=1$, and $\Phi_{k}^{(i, p)}=\emptyset$. This sequence $\Lambda_{(i, p)}=$ $\left\{\left\{\left(\Upsilon_{k}^{(i, p)}, d_{(j, k)}^{(i, p)}\right)\right\}_{j=1}^{n_{k}}\right\}_{k=0}^{s}$ is called the reducing algorithm of $p$ at $i$.

Note that for an unbounded $p \in P$, its reducing algorithm may not be finite. However, since this research is only interested in bounded $p \in P$, this next proof will show that the reducing algorithm constructed from such a $p$ is finite.

Theorem 4.24. For $i \in I$, and $p \in P$, if the following are satisfied:

- $T$ is a valid analytical task set.

- $p$ is bounded with respect to $(I, T, D)$.

then for $i \in I, \Lambda_{(i, p)}$ contains a finite number of choices. 


\subsection{Research Strategy Design Processes}

Proof. Let $i \in I$, and let $\Lambda_{(i, p)}=\left\{\left\{\left(\Upsilon_{k}^{(i, p)}, d_{(j, k)}^{(i, p)}\right)\right\}_{j=1}^{n_{k}}\right\}_{k=0}^{s}$ be the reducing algorithm of $p$ at $i$. Then since $p$ is bounded with respect to $(I, T, D)$, then $|T(i, p)|<\infty$ by definition of bounded. Then since $s \leq|T(i, p)|$, then $s$ is finite.

Since $p$ is bounded with respect to $(I, T, D)$, then $n_{k}=\left|\Phi_{k}^{(i, p)}\right|=\left|D_{\left(i, \Upsilon_{k}^{(i, p)}, p\right)}\right|$ is finite for $0 \leq k \leq s$ by definition of bounded. Therefore, there are $\sum_{k=0}^{s} n_{k}<\infty$ possible choices to consider.

Since $i \in I$ is arbitrary, then for any $i \in I, \Lambda_{(i, p)}$ contains a finite number of choices.

The next definition describes how to calculate the successors to a given knowledge state index after a given task list and experimental design have been chosen for consideration.

Definition 4.25. For $i=\left(i_{\mathscr{F}}, i_{\mathscr{G}}, i_{\mathscr{H}}\right) \in I, p \in P, S \subseteq T_{(i, p)}$, and $d \in D_{(i, S, p)}$, define

$$
\delta(i, p, S, d):=\left\{j: j \in I, j_{\mathscr{F}}=i_{\mathscr{F}} \cup y \text { for } y \in Y_{p, S, d}, j_{\mathscr{G}}=i_{\mathscr{G}}, j_{\mathscr{H}}=i_{\mathscr{H}}-\operatorname{cost}(d)\right\}
$$

where $Y_{p, S, d}=\left\{\bigcup_{F \in \vec{S}}\left\{f_{F}\right\}: f_{F} \in F\right.$ for each $\left.F \in \vec{S}\right\}$, and $i_{\mathscr{C}}$-cost $(d)$ is the change of resources from $i_{\mathscr{H}}$ after $d$ is performed. $\delta$ is called the transition function, and $\delta(i, p, S, d)$ is the successor list of $(i, p, S, d)$. Define $\Delta_{(i, p)}:=\{\delta(i, p, S, d):(S, d) \in$ $\left.\Lambda_{(i, p)}\right\}$ as the successor potential of $i$ using $p$.

A knowledge state index can either be a terminating index under $p$, where experimentation would stop, or an intermediate index under $p$. There are several types of terminating indexes, which this next set of definitions will describe. 


\subsection{Research Strategy Design Processes}

Definition 4.26. Let $i \in I$ and $p \in P$, and let $\Lambda_{(i, p)}=\left\{\left\{\left(\Upsilon_{k}^{(i, p)}, d_{(j, k)}^{(i, p)}\right)\right\}_{j=1}^{n_{k}}\right\}_{k=0}^{s}$ be the reducing algorithm of $p$ at $i$. If $\operatorname{pref}_{\text {projSat }}^{p}(i)=$ True, then $p$ is successful at $i$, which is called an F-Good terminating index under $p$. If $i$ is not an F-Good terminating index under $p$, and $\Upsilon_{0}^{(i, p)}=\emptyset$, then $p$ is unsuccessful at $i$ for logic reasons, which is

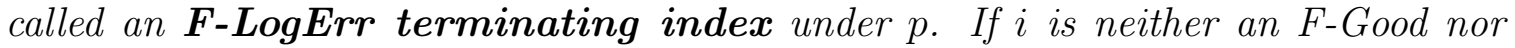
F-LogErr terminating index under $p$, and $\Phi_{k}^{(i, p)}=\emptyset$ for all $k \in\{0,1, \ldots, s\}$, then $p$ is unsuccessful at $i$ for resource reasons, which is called is an $\boldsymbol{F}$-ResErr terminating index under $p$. If $i$ is an F-Good terminating index, F-LogErr terminating index, or F-ResErr terminating index, then $i$ is a terminating index under $p$.

The type of an intermediate index is determined by the feedback from its successors, and the feedback from its successors is determined partially from the types of those successors. This next set of definitions describes this recursive process.

The process begins with the function that evaluates the successor list of the intermediate index.

Definition 4.27. The feedback evaluation function $\kappa: I \times P \rightarrow \overline{\mathbb{R}}$ evaluates the successor lists of intermediate indices.

$\kappa$ determines the type of the intermediate index.

Definition 4.28. Let $i \in I$ be an intermediate index under $p$. If $\kappa(i, p) \geq \operatorname{pref}_{\text {thresh }}^{p}(i)$, then $i$ is an $\boldsymbol{F - G o o d ~ i n t e r m e d i a t e ~ i n d e x ~ u n d e r ~} p$. If $\kappa(i, p)=-\infty$, then $i$ is an F-LogErr intermediate index under $p$. If $i$ is not an F-Good or F-LogErr intermediate index under $p$, then $i$ is an $\boldsymbol{F}$-ResErr intermediate index under $p$. 


\subsection{Research Strategy Design Processes}

Since intermediate indexes are evaluated with the terminating indexes that are contained in the same successor list, common names for their types must be defined. Definition 4.29. If a knowledge state index is either an F-Good intermediate index or F-Good terminating index under $p$, then it is $\boldsymbol{F}$-Good under $p$. If a knowledge state index is either an F-LogErr intermediate index or F-LogErr terminating index under $p$, then it is $\boldsymbol{F - L o g} \boldsymbol{E r r}$ under $p$. If a knowledge state index is either an F-ResErr intermediate index or F-ResErr terminating index under $p$, then it is $\boldsymbol{F}$-ResErr under $p$.

Once the common names are defined, a function can be constructed to evaluate each index in the successor list.

Definition 4.30. ise $: I \times P \rightarrow \overline{\mathbb{R}}$ is the state evaluation function. For $i \in I$ and $p \in P$, define

$$
\text { ise }(i, p):= \begin{cases}1 & \text { if } i \text { is F-Good under } p \\ -\infty & \text { if } i \text { is F-LogErr under } p \\ 0 & \text { if } i \text { is F-ResErr under } p\end{cases}
$$

ise $(i, p)$ can also be set to halt and flag the research construction process if an F-LogErr knowledge state index is encountered, in order to make sure such errors are manually addressed by the sponsor and designer. 


\subsection{Research Strategy Design Processes}

After each knowledge state index in the successor list is evaluated, their collective feedback value can be determined.

Definition 4.31. For $i \in I, p \in P$, where $i$ is an intermediate index under $p$, and $L \in \Delta_{(i, p)}$, define

$$
\operatorname{slfe}(i, p, L):= \begin{cases}\frac{\sum_{h \in L} i s e(h, p) \operatorname{pref}_{\text {weight }}^{p}(h)}{\sum_{h \in L} \operatorname{pref}_{\text {weight }}^{p}(h)} & \sum_{h \in L} \operatorname{pref}_{\text {weight }}^{p}(h) \neq 0 \\ 1 & \sum_{h \in L} \operatorname{pref}_{\text {weight }}^{p}(h)=0\end{cases}
$$

slfe is called is the successor list feedback evaluation function.

The feedback evaluation function then determines the greatest possible collective feedback value for the intermediate index.

Definition 4.32. For $i \in I$ and $p \in P$, where $i$ is an intermediate index under $p$, define

$$
\kappa(i, p):=\max \left(\left\{\operatorname{slfe}(i, p, L): L \in \Delta_{(i, p)}\right\}\right)
$$

This next function returns the preferred choice for an intermediate index, which is a choice that fits the criteria of the reducing algorithm, and is the first choice in the algorithm that is not eliminated by feedback.

Definition 4.33. Let $i \in I$ and $p \in P$, such that $i$ is an intermediate index under $p$, 


\subsection{Research Strategy Design Processes}

and let $\Lambda_{(i, p)}=\left\{\left\{\left(\Upsilon_{k}^{(i, p)}, d_{(j, k)}^{(i, p)}\right)\right\}_{j=1}^{n_{k}}\right\}_{k=0}^{s}$ be the reducing algorithm of $p$ at $i$. Define

$$
p c(i, p):=\left(\Upsilon_{k}^{(i, p)}, d_{(j, k)}^{(i, p)}\right)
$$

where $\operatorname{slfe}\left(i, p, \delta\left(i, p, \Upsilon_{k}^{(i, p)}, d_{(j, k)}^{(i, p)}\right)\right) \geq \min \left(\operatorname{pref}_{\text {thresh }}^{p}(i), \kappa(i, p)\right)$, and there does not exist a $\left(\Upsilon_{s}^{(i, p)}, d_{(r, s)}^{(i, p)}\right)$ such that slfe $\left(i, p, \delta\left(i, p, \Upsilon_{r}^{(i, p)}, d_{(r, s)}^{(i, p)}\right)\right) \geq \min \left(\operatorname{pref}_{\text {thresh }}^{p}(i), \kappa(i, p)\right)$ and either $r<k$ or $r=k, s \leq j . p c(i, p)$ is called the preferred choice of $p$ at $i$.

This next function, using the previously described methods, returns the planned move for a given knowledge state index, according to a given sponsor-designer preference function list.

Definition 4.34. Let $p \in P$. For $i \in I$ define

$$
\theta(i, p):= \begin{cases}p c(i, p) & \text { if } i \text { is an intermediate index under } p \\ (\text { FinishGood,Finish }) & \text { if } i \text { is an F-Good terminating index under } p \\ (\text { FinishResErr,Finish) } & \text { if } i \text { is an F-ResErr terminating index under } p \\ (\text { FinishLogErr,Finish) } & \text { if } i \text { is an F-LogErr terminating index under } p\end{cases}
$$

where Finish is a placeholder when no design is needed, FinishGood is a command to finish experimentation as a success, FinishResErr is a command to finish experimentation as a partial success, and FinishLogErr is an error command stating that $p$ could not find relevant analytical tasks to address $i$. Define $\theta_{p}:=\{\theta(i, p)\}_{i \in I}$. $\theta_{p}$ is called the research strategy of $p$. Define $\Theta_{P}:=\left\{\theta_{p}\right\}_{p \in P} . \Theta_{P}$ is called the research 


\subsection{ARC-RSM Partial Orders}

strategy set for $P$.

For $i \in I$, if $\theta(i, p)$ is F-Good, then $p$ is successful (goal attaining, within budget, logically defendable, reproducable) at meeting the project requirements starting at $i$.

\subsection{ARC-RSM Partial Orders}

This section discusses and demonstrates partial orders that can be used to partially order $I$ in terms of $P$. Those partial orders will then be used to show useful properties of this methodology.

Definition 4.35. For $p \in P$ and $i \in I, c=\left(c_{1}, c_{2}\right) \in \Lambda_{(i, p)}$ is a potential choice for $i$ by $p$ if $c_{2} \in D_{\left(i, c_{1}, p\right)}$.

Definition 4.36. For $p \in P, \omega^{p} \subseteq I \times I$ is a relation called a research strategic arrangement of $I$ such that for $i_{j}, i_{k} \in I$, if $\left(i_{j}, i_{k}\right) \in \omega^{p}$, there exists a potential choice $c=\left(c_{1}, c_{2}\right) \in \Lambda_{(i, p)}$ such that $\left(i_{j}, i_{m}\right) \in \omega^{p}$ if and only if $i_{m} \in \delta\left(i_{j}, p, c_{1}, c_{2}\right)$. For $i_{j}, i_{k} \in I,\left(i_{j}, i_{k}\right) \in \omega^{p}$ can be written as $i_{j} \omega^{p} i_{k}$. Let $\Omega_{p}$ be the set of all research strategic arrangements for a given $p \in P$, and let $\Omega_{P}$ be the set of all research strategic arrangements for each $p \in P$.

Definition 4.37. Given a sequence $\left\{u_{j}\right\}_{j=1}^{m} \subseteq I$, if there exists a $p \in P$ and $\omega^{p} \subseteq I \times I$ such that $u_{j} \omega^{p} u_{j+1}$ for $j \in\{1, \cdots, m-1\}$, then $\left\{u_{j}\right\}_{j=1}^{m} \subseteq I$ is a considered path from $u_{1}$ to $u_{m}$ through $\omega^{p}$. For $a, b \in I$ and $p \in P, b$ is considerable from a through $\omega^{p}$ if there exists a considered path from a to $b$ through $\omega^{p}$. 


\subsection{ARC-RSM Partial Orders}

This section will later show that considered paths are, in fact, paths.

Definition 4.38. For $a, b \in I$ and $\omega^{p} \in \Omega_{P}, a \preccurlyeq \omega_{\omega^{p}} b$ if and only if $b$ is considerable from a through $\omega^{p}$.

Lemma 4.39. Let $T$ be a valid analytical task set. For distinct $a, b \in I$ and $p \in P$, if $a \preccurlyeq \omega^{p} b$, then $a_{\mathscr{F}} \subset b_{\mathscr{F}}$.

Proof. Since $a \preccurlyeq_{\omega^{p}} b$, then there exists a considered path $\left\{u_{j}\right\}_{j=1}^{m} \subseteq I$ from $u_{1}=a$ to $u_{m}=b$ through $\omega^{p}$. Therefore, since $T$ is a valid analytical task set, then $\left(u_{j}\right)_{\mathscr{F}} \subset$ $\left(u_{j+1}\right)_{\mathscr{F}}$ for each $j \in\{1,2, \ldots m-1\}$. Therefore, $a_{\mathscr{F}}=\left(u_{1}\right)_{\mathscr{F}} \subset\left(u_{m}\right)_{\mathscr{F}}=b_{\mathscr{F}}$, so $a_{\mathscr{F}} \subset b_{\mathscr{F}}$.

Theorem 4.40. Let $T$ be a valid analytical task set. Then $\preccurlyeq \omega^{p}$ is a partial order.

Proof. For $a \in I, a$ is always considerable from $a$ using the trivial sequence $\{a\}$, so $\preccurlyeq \omega^{p}$ is reflexive.

Assume that $a, b \in I$ such that $a \preccurlyeq \omega^{p} b$, and $a \neq b$. Therefore, $b$ is considerable from $a$ through $\omega^{p}$. Therefore, since $p \in P$, then by Lemma $4.39, a_{\mathscr{F}} \subset b_{\mathscr{F}}$. Therefore, $b_{\mathscr{F}} \not \subset a_{\mathscr{F}}$, so by Lemma $4.39, b \aleph_{\omega^{p}} a$. Therefore, since $a, b \in I$ are arbitrary, $\preccurlyeq \omega^{p}$ is antisymmetric.

Assume that $a, b, c \in I$ such that $a \preccurlyeq \omega^{p} b$ and $b \preccurlyeq_{\omega^{p}} c$. Since $a \preccurlyeq_{\omega^{p}} b$ and $b \preccurlyeq_{\omega^{p}} c$, then $b$ is considerable from $a$ through $\omega^{p}$ and $c$ is considerable from $b$ through $\omega^{p}$. Therefore, there exist sequences $\left\{u_{j}\right\}_{j=1}^{m},\left\{v_{k}\right\}_{k=1}^{n} \subseteq I$ such that $u_{1}=a, u_{m}=v_{1}=$ $b, v_{n}=c,\left\{u_{j}\right\}_{j=1}^{m}$ is a considered path from $a$ to $b$ through $\omega^{p}$, and $\left\{v_{k}\right\}_{k=1}^{n}$ is a 


\subsection{ARC-RSM Partial Orders}

considered path from $b$ to $c$ through $\omega^{p}$. If $\left\{u_{j}\right\}_{j=1}^{m}$ and $\left\{v_{k}\right\}_{k=1}^{n}$ intersect at an index $d \in I$, then $b \preccurlyeq_{\omega^{p}} d$ and $d \preccurlyeq \omega^{p} b$, so since $\preccurlyeq_{\omega^{p}}$ is antisymmetric, $d=b$. Therefore, $\left\{u_{j}\right\}_{j=1}^{m}$ and $\left\{v_{k}\right\}_{k=1}^{n}$ can be connected into a new sequence $\left\{w_{l}\right\}_{l=1}^{m+n-1}$, which is a considered path from $a$ to $c$ through $\omega^{p}$. Therefore, $c$ is considerable from $a$ through $\omega^{p}$, so $a \preccurlyeq \omega^{p} c$.

Therefore, $\preccurlyeq \omega^{p}$ is transitive.

Since $\preccurlyeq_{\omega^{p}}$ is reflexive, antisymmetric, and transitive, it is a partial order.

Definition 4.41. For $a, b \in I, a \preccurlyeq \Omega_{p} b$ if and only if there exists $a \omega^{p} \in \Omega_{p}$ such that $a \preccurlyeq \omega^{p} b$.

Theorem 4.42. Let $T$ be a valid analytical task set. Then $\preccurlyeq \Omega_{p}$ is a partial order.

Proof. For $a \in I, a$ is always considerable from $a$ using the trivial sequence $\{a\}$, so $\preccurlyeq \Omega_{p}$ is reflexive.

Assume that $a, b \in I$ such that $a \preccurlyeq \Omega_{p} b$, and $a \neq b$. Therefore, there must exist a $p \in P$ such that $b$ is considerable from $a$ through $\omega^{p}$. Therefore, since $p \in P$, by Lemma 4.39, $a_{\mathscr{F}} \subset b_{\mathscr{F}}$. Therefore, $b_{\mathscr{F}} \not \subset a_{\mathscr{F}}$, so by Lemma $4.39, b \aleph_{\Omega_{p}} a$. Therefore, since $a, b \in I$ are arbitrary, $\preccurlyeq \Omega_{p}$ is antisymmetric.

Assume that $a, b, c \in I$ such that $a \preccurlyeq \Omega_{p} b$ and $b \preccurlyeq \Omega_{p} c$. Since $a \preccurlyeq \Omega_{p} b$ and $b \preccurlyeq \Omega_{p} c$, then there exist $\omega_{1}^{p}, \omega_{2}^{p} \in \Omega_{p}$, such that $b$ is considerable from $a$ through $\omega_{1}^{p}$ and $c$ is considerable from $b$ through $\omega_{2}^{p}$. Therefore, there exist sequences $\left\{u_{j}\right\}_{j=1}^{m},\left\{v_{k}\right\}_{k=1}^{n} \subseteq I$ such that $u_{1}=a, u_{m}=v_{1}=b, v_{n}=c,\left\{u_{j}\right\}_{j=1}^{m}$ is a considered path from $a$ through $\omega_{1}^{p}$, and $\left\{v_{k}\right\}_{k=1}^{n}$ is a considered path from $b$ through $q$. If $\left\{u_{j}\right\}_{j=1}^{m}$ and $\left\{v_{k}\right\}_{k=1}^{n}$ 


\subsection{ARC-RSM Partial Orders}

intersect at an index $d \in I$, then $b \preccurlyeq \Omega_{p} d$ and $d \preccurlyeq \Omega_{p} b$, so since $\preccurlyeq \Omega_{p}$ is antisymmetric, $d=b$. Therefore, there exists a $\omega_{3}^{p} \in \Omega_{p}$ such that $\left\{\left(u_{j}, u_{j+1}\right)\right\}_{j=1}^{m-1},\left\{\left(v_{k}, v_{k+1}\right)\right\}_{k=1}^{n-1} \subseteq$ $\omega_{3}^{p}$. Therefore, $c$ is considerable from $a$ through $\omega_{3}^{p}$, so $a \preccurlyeq \Omega_{p} c$. Therefore, $\preccurlyeq \Omega_{p}$ is transitive.

Since $\preccurlyeq \Omega_{p}$ is reflexive, antisymmetric, and transitive, it is a partial order.

This next theorem establishes that the logical structure a sponsor-designer preference function list generates is a tree (there is at most one path between any two points). It also shows that considered paths are, in fact, paths.

Lemma 4.43. For $a, b \in I, p \in P$, and $\omega^{p} \in \Omega_{p}$, if $a \preccurlyeq \omega^{p} b$, then $a \preccurlyeq \Omega_{p} b$.

Proof. Since $a \preccurlyeq \omega^{p} b$ and $\omega^{p} \in \Omega_{p}$, then $a \preccurlyeq \Omega_{p} b$ by definition of $\preccurlyeq \Omega_{p}$.

Theorem 4.44. Let $T$ be a valid analytical task set. For distinct $a, b, c \in I$ and $p \in P$, if $a \preccurlyeq \omega_{\omega^{p}} b, a \preccurlyeq_{\omega^{p}} c, b \npreceq_{\omega^{p}} c$, and $c \aleph_{\omega^{p}} b$, then there does not exist $a d \in I$ such that $b \preccurlyeq \Omega_{p} d$ and $c \preccurlyeq \Omega_{p} d$.

Proof. Since $a \preccurlyeq \omega^{p} b$ and $a \preccurlyeq \omega^{p} c$, where $a, b, c$ are distinct, then there exist distinct sequences $\left\{u_{j}\right\}_{j=1}^{m},\left\{v_{k}\right\}_{k=1}^{n} \subseteq I$ such that $u_{1}=v_{1}=a, u_{m}=b, v_{n}=c,\left\{u_{j}\right\}_{j=1}^{m}$ is a considered path from $a$ to $b$ through $\omega^{p}$, and $\left\{v_{k}\right\}_{k=1}^{n}$ is a considered path from $a$ to $c$ through $\omega^{p}$. If $u_{m}=b \in\left\{v_{k}\right\}_{k=1}^{n}$, then $b \preccurlyeq \omega^{p} c$, which contradicts an initial assumption that $b \npreceq_{\omega^{p}} c$. Therefore, there exists a $k \in\{1,2, \cdots, m\}$ such that $u_{k} \neq v_{k}$. Also, since $u_{1}=v_{1}=a$, then there exists a $k \in\{1,2, \cdots, m\}$ such that $u_{k}=v_{k}$. Therefore, choose $k \in\{1,2, \cdots, m\}$ such that $u_{k} \neq v_{k}$. 


\subsection{ARC-RSM Partial Orders}

Assume there exists a $d \in I$ such that $u_{k} \preccurlyeq \Omega_{p} d$ and $v_{k} \preccurlyeq \Omega_{p} d$. Since $u_{k} \neq v_{k}$, and $u_{1}=v_{1}=a$, then $k>1$. If $u_{k-1}=v_{k-1}$, then $u_{k}, v_{k}$ are both successors of $u_{k-1}$, so $u_{k}, v_{k}$ have the same amount of resources, and are the results of the same analytical tasks. Therefore, since $u_{k} \neq v_{k}$, then they must differ in the results of the tests. Therefore, if $u_{k} \preccurlyeq \Omega_{p} d$ and $v_{k} \preccurlyeq \Omega_{p} d$, then by Lemma 4.39 , $\left(u_{k}\right)_{\mathscr{F}} \subset d_{\mathscr{F}}$ and $\left(v_{k}\right)_{\mathscr{F}} \subset d_{\mathscr{F}}$, so $\left(u_{k}\right)_{\mathscr{F}} \cup\left(v_{k}\right)_{\mathscr{F}} \subset d_{\mathscr{F}}$, so $d_{\mathscr{F}}$ must have contain two facts from the same output of a $\tau \in T$, which contradicts the initial assumption that $T$ is a valid analytical task set. Therefore, $u_{k-1}=v_{k-1}$ must be false, so $u_{k-1} \neq v_{k-1}$.

Therefore, if $u_{t} \neq v_{t}$ for $t \in\{2, \cdots, m\}$, and $u_{t} \preccurlyeq \Omega_{p} d$ and $v_{t} \preccurlyeq \Omega_{p} d$, then $u_{t-1} \neq v_{t-1}$. Also, if $u_{t} \preccurlyeq \Omega_{p} d$ for $t \in\{2, \cdots, m\}$, then $u_{s} \preccurlyeq \Omega_{p} d$ for $s \in\{1, \cdots, t-1\}$, by Theorem 4.42 and Lemma 4.43 . Similarly, if $v_{t} \preccurlyeq \Omega_{p} d$ for $t \in\{2, \cdots, n\}$, then $v_{s} \preccurlyeq \Omega_{p} d$ for $s \in\{1, \cdots, t-1\}$, by Theorem 4.42 and Lemma 4.43 .

Therefore, for $k \in\{2, \cdots, m\}$ such that $u_{k} \neq v_{k}$, and $t \in k, \cdots, m$, if $u_{k} \preccurlyeq \Omega_{p} d$ and $v_{t} \preccurlyeq \Omega_{p} d$, then $u_{2} \preccurlyeq \Omega_{p} d$ and $v_{2} \preccurlyeq \Omega_{p} d$, so $u_{1} \neq v_{1}$, which contradicts $u_{1}=v_{1}=a$. Therefore, since $u_{m}=b$ and $v_{n}=c$, there does not exist a $d \in I$ such that $b \preccurlyeq \Omega_{p} d$ and $c \preccurlyeq \Omega_{p} d$.

The next proofs show that the reducing algorithm can be evaluated in finite time.

Lemma 4.45. Let $T$ be a valid analytical task set. Then for each $i \in I$, there exists a finite length $l$ such that any considered paths to $i$ through any $\omega^{p} \in \Omega_{p}$ are bounded by $l$.

Proof. Let $i \in I$. 


\subsection{ARC-RSM Partial Orders}

Let $l=\left|i_{\mathscr{F}}\right|$, which is finite by definition of $\mathscr{F}$. Therefore, since $T$ is a valid analytical task set, then by Lemma 4.39, any considered path to $i$ can have length at most $l<\infty$.

Since $i \in I$ is arbitrary, then for each $i \in I$, there exists a finite length $l$ such that any considered paths to $i$ through any $\omega^{p} \in \Omega_{p}$ are bounded by $l$.

Lemma 4.46. Let $p \in P$ be bounded. Then for each $i \in I$, there exists a finite length $l$ such that any considered paths from $i$ through any $\omega^{p} \in \Omega_{p}$ are bounded by $l$.

Proof. Let $i \in I$.

By definition of $\mathscr{H}$, the number of types of resource are finite. Therefore, since $p$ is bounded, there exists a finite subset $D_{\min } \subseteq D_{p}$ such that $\operatorname{cost}(d)>0$ for all $d \in D_{\text {min }}$, and there does not exist a $d_{1} \in D_{p}$ such that $\operatorname{cost}\left(d_{1}\right)<\operatorname{cost}\left(d_{2}\right)$ for some $d_{2} \in D_{\min }$. Therefore, there exists a $d_{\text {min }} \in D_{\text {min }}$ such that $i_{\mathscr{H}} / \operatorname{cost}\left(d_{\text {min }}\right) \geq i_{\mathscr{H}} / \operatorname{cost}\left(d_{0}\right)$ for all $d_{0} \in D_{\text {min }}$, and therefore $i_{\mathscr{H}} / \operatorname{cost}\left(d_{\text {min }}\right) \geq i_{\mathscr{H}} / \operatorname{cost}\left(d_{0}\right)$ for all $d_{0} \in D$. Therefore, any considered path from $i$ can be at most length $i_{\mathscr{H}} / \operatorname{cost}\left(d_{\min }\right)$.

Let $l=i_{\mathscr{H}} / \operatorname{cost}\left(d_{\text {min }}\right)$. Since $d_{\text {min }} \in D_{\text {min }}$, then $\operatorname{cost}\left(d_{\text {min }}\right)>0$. And by definition of $\mathscr{H}, i_{\mathscr{H}}$ has a finite amount of resources. Therefore, any considered path from $i$ can be at most length $l<\infty$.

Since $i \in I$ is arbitrary, then for each $i \in I$, there exists a finite length $l$ such that any considered paths from $i$ through any $\omega^{p} \in \Omega_{p}$ are bounded by $l$.

Lemma 4.47. For $i \in I$, and $p \in P$, if the following are satisfied:

- $T$ is a valid analytical task set. 


\subsection{ARC-RSM Partial Orders}

- $p$ is bounded with respect to $(I, T, D)$.

then for $i \in I$, there are a finite number of maximal considered paths from $i$ through $\Omega_{p}$.

Proof. Let $i \in I$. Define $J_{i}=\left\{j: j \in I, i \omega^{p} j\right.$ for some $\left.\omega^{p} \in \Omega_{p}\right\}$. By Theorem 4.24, $\Lambda_{(i, p)}$ contains a finite number $(N)$ of choices. Since $p$ is bounded, then for each $c:=\left(c_{1}, c_{2}\right) \in \Lambda_{(i, p)}, \delta\left(i, p, c_{1}, c_{2}\right)$ is finite. Therefore, $\left|J_{i}\right| \leq|N| \cdot \max \left\{\left|\delta\left(i, p, c_{1}, c_{2}\right)\right|:\right.$ $\left.\left(c_{1}, c_{2}\right) \in \Lambda_{(i, p)}\right\}<\infty$, so $\left|J_{i}\right|<\infty$. Therefore, since $i \in I$ is arbitrary, then for $j \in J_{i}$, $\left|J_{j}\right|<\infty$, and $\left|J_{j^{\prime}}\right|<\infty$ for $j^{\prime} \in J_{j}$, and so on. Therefore, for each $0<k<\infty$, there exists a finite $M_{k}=\max \left\{\left|\delta\left(j, p, c_{1}, c_{2}\right)\right|: j \in I,\left(c_{1}, c_{2}\right) \in \Lambda_{(j, p)}, \operatorname{dist}_{\preccurlyeq \Omega_{p}}(i, j)=k-1\right\}$, where $\operatorname{dist}_{\preccurlyeq \Omega_{p}}(i, j)$ is the minimum length of a path from $i$ to $j$ using $\preccurlyeq \Omega_{p}$.

By Lemma 4.46, there exists a finite length $l$ such that all considered paths from $i$ through $\Omega_{p}$ have length at most $l$. Therefore, there are at most $\prod_{k=1}^{l}\left|M_{k}\right|<\infty$ considered paths from $i$ through $\Omega_{p}$.

Because there are a finite number of considerable paths from $i$, each of finite length, exploration of all paths from $i$ can be accomplished in finite time. 


\section{5}

\section{Sponsor-Designer specification of}

\section{ARC-RSM}

\subsection{Preliminary Sponsor-Designer Discussions}

Design of Experiments, and therefore ARC-RSM, is based on statistical methods and being able to get needed specification information from the sponsor about what statistical methods are appropriate for the current project. In order to make design choices, a designer must first have answers to the following questions:

- What is the goal?

- What information would satisfy this goal? 


\subsection{Preliminary Sponsor-Designer Discussions}

This information gives at least a basic pseudocode about how to program the project satisfaction function $\operatorname{pref}_{\text {projSat }}^{p}$.

The set of analytical tasks, $T$, needs to contain tasks that can produce information that would satisfy the goal. Because of this, more questions must be answered:

- What relevant information is initially available?

- What available analytical tasks would be able to produce the needed information?

- What additional information do the required analytical tasks need?

- What available analytical tasks would be able to produce the information needed for those analytical tasks?

This particular conversation helps determine a conceptual breakdown of the overall goal into stages that will be useful in making individual decisions. When breaking down a goal conceptually, the key idea is to decompose the problem into pieces that can each be completed in one step.

Once there is an idea about what analytical tasks are needed, the set of experimental designs, $D$, needs to have affordable experimental designs that can support those tasks. Therefore, more questions must be answered:

- What resources (including time) are available?

- What are the available testing conditions? 


\subsection{Sponsor-Designer Task and Design Selections}

- What experimental designs of interest, if any, are able to support the kinds of needed analytical tasks, and can fit the testing conditions (at all)?

This conversation is important for not only determining what can be done, but for helping get an idea for how complex the project might really be, especially with respect to what can be done within an available budget. The project goal might be simplified, and/or the resources might be increased.

At this point, there will be a conceptual understanding of what needs to be done, and what can be done. This understanding will get more and more precise as further specifications are made.

\subsection{Sponsor-Designer Task and Design Selections}

When specifying analytical tasks, the following questions must be answered:

- What level of information will be considered necessary for completing the project?

- What system variables are of greatest interest?

- What system interactions are of greatest interest, and to what degree?

- What information can be sacrificed in order to afford completing experimentation?

- How can non-critical analytical tasks be prioritized? 


\subsection{Sponsor-Designer Feedback Specifications}

These questions will determine the analytical task specification functions, although they can be changed later if they are judged to be inadequate during research strategy construction.

When choosing experimental designs, the following questions must be answered:

- What is considered more important: cost, or quality?

- How will that decision be influenced by the content of knowledge state indexes?

- How will those previous two decisions be influenced by differing amounts of available resources?

These questions will determine the experimental design preference ordering function, although like the analytical task specification functions, it can be changed later if it is judged to be inadequate during research strategy construction.

\subsection{Sponsor-Designer Feedback Specifications}

When compromise occurs in ARC-RSM, it means that the ideal solution is not available. In order to get an initial idea about how compromise should occur, these questions must be answered:

- In general, what level of compromise is considered acceptable?

- Where would compromise be more acceptable? 


\subsection{Sponsor-Designer Feedback Specifications}

The index priority weight function is used to determine the importance of a given successor. When determining the index priority weight function, the following questions must be answered:

- What possible knowledge state indexes represent scenarios of greatest interest?

- Under what circumstances should a unsuccessful successor be "settled for" by its parent?

The feedback compromise threshold function is used to provide the standard used to determine if the feedback is good enough, or whether the planned move needs to make compromises. When determining the feedback compromise threshold function, the following questions must be answered:

- Should the index priority weight function be incorporated in deciding the quality of feedback needed to accept a planned move?

- If so, how?

- Should knowledge state index information be incorporated in deciding the quality of feedback needed to accept a planned move from that knowledge state index?

- If so, how?

The index priority weight function and feedback compromise threshold function are there to incorporate where the sponsor is willing to compromise for the sake of 


\subsection{Sponsor-Designer Feedback Specifications}

being able to affordably gain the most valuable information from this project. These functions, along with the other preference functions, can be adjusted if they are judged to be inadequate during research strategy construction. 
6

\section{Applications and Demonstrations}

\subsection{Summary of Simulation Code and Testing Meth- ods}

This section describes the testing of the decision-making process of ARC-RSM, as determined by the preference functions. To do this, an example is derived from Karnik, Gaitonde, and Davim (45):

Example 6.1. A manufacturing company is having a quality issue with the parts they are manufacturing having exit burrs. 


\subsection{Summary of Simulation Code and Testing Methods}

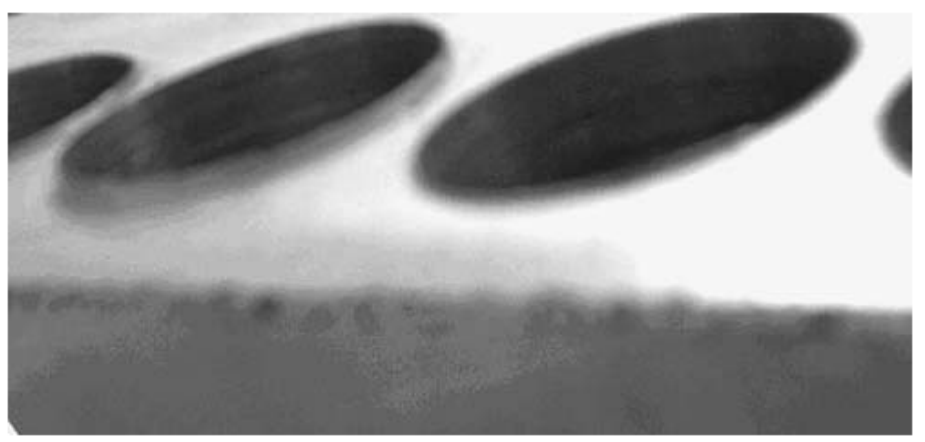

Figure 6.1: Exit burrs observed during drilling.

A company representative (the sponsor) has contacted a statistician familiar with experimental design (the designer) to help plan research to determine how burrs are influenced by characteristics of the manufacturing process.

ARC-RSM is itself algorithmic, so the code used to enact it is a literal interpretation of the mathematical structures, with additional code for specification of the preference functions, user input, and graphical output. The code does not only perform ARC-RSM, but takes in user input, and presents the results in a visually interesting and informative manner that is compatible with LaTeX.

Since the algorithm is tree-based (see Theorem 4.44), it is implemented through recursion. Each node develops its own respective subtree based on its own reducing algorithm. If a knowledge state index's successors meets the feedback conditions, the successors' respective subtrees are then attached to the current node, and the current knowledge state index would now have its own respective tree. In the case where a knowledge state index is a terminating index, the tree is just the knowledge state index's node by itself. Once this process is completed, this tree represents 


\subsection{Summary of Simulation Code and Testing Methods}

the constructed research strategy. The code is distinguished into several categories: interface scripts, main functions, preference functions, conversion functions, logical functions, sorting functions, display functions, and miscellaneous functions, in total constituting 4425 lines of code.

Interface scripts are scripts like the main menu, and anything that would take in user data and regulate execution of the functions. The main functions are functions like the preferred choice function, and any function of ARC-RSM that would not change based upon the project. Preference functions are those functions that are specified during the sponsor-designer interactions. Conversion functions convert one data type into another. Logical functions are simply those functions that return Boolean values that are not main or preferred functions, such as a function to determine if a task list has nonlinear terms. Sorting functions sort the data, such as arranging the outputs of analytical tasks to insert into successors, and/or creating reference points to better access the outputs of Matlab's statistical functions. Display functions display information in a way that users can understand. The miscellaneous functions are those functions that do not fit into any of the previous categories.

There are five different, interconnected phases showing the applicability, testability, and adaptability of the methodology:

1. The first phase reflects the initial sponsor's viewpoint of how experimentation should proceed, to see if a research strategy can be formed.

2. The second phase extends the first phase by trying to find a minimal strategy 


\subsection{Summary of Simulation Code and Testing Methods}

meeting the initial preferences.

3. The third phase is a refinement of the preferences to find a better working research strategy.

4. The fourth phase demonstrates what happens when a project's satisfaction requirements are too strict.

5. The fifth phase tests how well a research strategy can react to unexpected loss of resource.

Each of the first four phases uses four different functions (simple polynomials, for ease of comparison) as test cases, with different levels of interaction, significance, and polynomial order:

1. The first function is a complete quadratic function including CuttingSpeed, FeedRate, and PointAngle. All quadratic and linear terms should make it into the response function estimation.

2. The second function is a linear function including CuttingSpeed, FeedRate, and PointAngle. All linear terms, and no quadratic terms, should make it into the response function estimation.

3. The third function is a complete quadratic function including CuttingSpeed, FeedRate, but not PointAngle. All quadratic and linear terms including CuttingSpeed and FeedRate, but not PointAngle, should make it into the response function estimation. 


\subsection{Phase 1 - Initial Phase}

4. The fourth function does not include Cutting Speed, FeedRate, nor Point Angle. No terms including CuttingSpeed, FeedRate, or PointAngle should make it into the response function estimation. Ideally, they should all be screened out.

The last phase uses the first function, while changing the amount of resources during experimentation in order to observe how the algorithm is able to adapt to those changes.

\subsection{Phase 1 - Initial Phase}

\subsubsection{Preliminary Sponsor-Designer Discussions}

The designer goes over the following questions with the sponsor:

Question: What is the goal?

The sponsor wants to be able to predict the size of exit burrs created during manufacturing.

Question: What information would satisfy this goal?

The sponsor wants to know how exit burrs are affected by the manufacturing process.

Question: What relevant information is initially available? 


\subsection{Phase 1 - Initial Phase}

The sponsor says that cutting speed, feed rate, and the point angle are the significant influences of interest.

Question: What available analytical tasks would be able to produce the needed information?

The designer concludes that since the sponsor wants to be able to predict burr size, a response function is needed for that element in terms of the significant influences of interest. Therefore, regression is needed.

Question: What additional information do the required analytical tasks need?

The sponsor has provided cutting speed, feed rate, and the point angle as the significant influences of interest. Therefore, the designer decides that the variables for those elements (CuttingSpeed, FeedRate, and PointAngle respectively) should be screened before or during the modeling process.

Question: What available analytical tasks would be able to produce the information needed for those analytical tasks?

According to the designer, there should be significance tests included in the modeling process. These may be done before and/or during the regression.

Question: What resources (including time) are available?

The sponsor says that time is not a constraint, but there are the following limita- 


\subsection{Phase 1 - Initial Phase}

tions:

- Each run costs 1 unit of MaterialSample

- There are 90 units of MaterialSample

Question: What are the available testing conditions?

The sponsor provides the following information:

- CuttingSpeed $(C S)$ can be between $20-50 \mathrm{~m} / \mathrm{min}$

- FeedRate (FR) can be between $10-20 \mathrm{~mm} / \mathrm{rev}$

- PointAngle $(P A)$ can be between $0-45$ degrees

Question: What experimental designs of interest, if any, are able to support the kinds of needed analytical tasks, and can fit the testing conditions (at all)?

Since the design must perform regression within a closed and finite space, the experimental designer is interested in the $2^{k}$ factorial design with either 2 or 3 replications.

\subsubsection{Sponsor-Designer Task and Design Selections}

Question: What level of information will be considered necessary for completing the project? 


\subsection{Phase 1 - Initial Phase}

The sponsor wants an approximation of the response function for BurrSize, and has no preference towards its form.

Question: What system variables are of greatest interest?

The sponsor is interested in CuttingSpeed the most, then FeedRate, and then PointAngle.

Question: What system interactions are of greatest interest, and to what degree?

The sponsor does not have any preferences in terms of interactions.

Question: What information can be sacrificed in order to afford completing experimentation?

The sponsor is interested in CuttingSpeed the most, then FeedRate, and then PointAngle. Therefore, testing for PointAngle significance has the lowest priority, then FeedRate, with testing for Cutting Speed being considered essential.

Question: How can non-critical analytical tasks be prioritized?

The sponsor wants an approximation of the response function for BurrSize, and has no preference towards its form. Therefore, the sponsor and designer agree on the following prioritization, from highest priority to lowest:

- analyze effect of CuttingSpeed on BurrSize 


\subsection{Phase 1 - Initial Phase}

- analyze effect of FeedRate on BurrSize

- analyze effect of PointAngle on BurrSize

Question: What is considered more important: cost, or quality?

The sponsor wants the best affordable quality available at each stage.

Question: How will that decision be influenced by the content of knowledge state indexes?

The sponsor does not want the decision regarding cost vs. quality to be affected by the current state of knowledge unless it is shown to be not affordable.

Question: How will those previous two decisions be influenced by differing amounts of available resources?

The sponsor decides that regardless of the amount of available resources, higher quality experimental designs should be preferred over cheaper ones.

\subsubsection{Sponsor-Designer Feedback Specifications}

Question: In general, what level of compromise is considered acceptable?

The sponsor wants a response function appromimation of BurrSize in terms of at least CuttingSpeed, and will compromise anywhere else.

Question: Where would compromise be more acceptable? 


\subsection{Phase 1 - Initial Phase}

The sponsor wants an attempt to model a response function for BurrSize in terms of at least Cutting Speed, and will not compromise on that, but will compromise for anything else.

Question: What possible knowledge state indexes represent scenarios of greatest interest?

The sponsor does not care what kind of response function is returned, wants to keep an eye out for scenarios where no response function can be generated.

Question: Under what circumstances should a unsuccessful successor be "settled for" by its parent?

The sponsor wants some sort of response function for BurrSize, so wants complete success from feedback; at least to begin with.

Question: Should the index priority weight function be incorporated in deciding the quality of feedback needed to accept a planned move? If so, how?

The sponsor and designer agree to use equal weighting for each outcome, with complete success from feedback as the feedback criteria.

Question: Should knowledge state index information be incorporated in deciding the quality of feedback needed to accept a planned move from that knowledge state index? If so, how? 


\subsection{Phase 1 - Initial Phase}

The sponsor and designer agree on complete success from feedback as the feedback criteria for each knowledge state index.

\subsubsection{Specifications Expressed Formally by Designer for Phase 1}

Number of samples: 90

\section{Project Satisfaction Condition:}

All predictors screened out, or response function constructed

\section{Task Specification Function:}

If potential predictors have not gone through screening (in order of preference, greatest to least):

screen(BurrSize $(C S, F R, P A))$

screen $(\operatorname{BurrSize}(C S, F R))$

screen(BurrSize $(C S))$

If potential predictors have gone through screening (in order of preference, greatest to least):

quadraticmodel(BurrSize(screened predictors)), linearmodel(BurrSize(screened predictors)), 


\subsection{Phase 1 - Initial Phase}

linearmodel(BurrSize(screened predictors - screened predictor of least priority)) $\vdots$

linearmodel(BurrSize(highest priority screened variable)),

\section{Experimental Design Preference Ordering Function:}

For screening (in order of preference, greatest to least):

Full Factorial Design, 3 replications

Full Factorial Design, 2 replications

For linear modeling (in order of preference, greatest to least):

Full Factorial Design, 3 replications

Full Factorial Design, 2 replications

For quadratic modeling:

Central Composite Design

maximum number of center points $=(\text { number of screened predictors }+2)^{*} 2$

minimum number of center points $=$ number of screened predictors +4

maximum number of factorial points $=3$

minimum number of factorial points $=2$

maximum number of axial points $=3$

minimum number of axial points $=2$ 


\subsection{Phase 1 - Initial Phase}

start with maximum values, reduce number of center points first, then number of axial points, then number of factorial points 


\subsubsection{Constructed Research Strategy - Initial Phase}

Figure 6.2 is a visual representation of the F-Good research strategy, determined from Section 6.2.4:

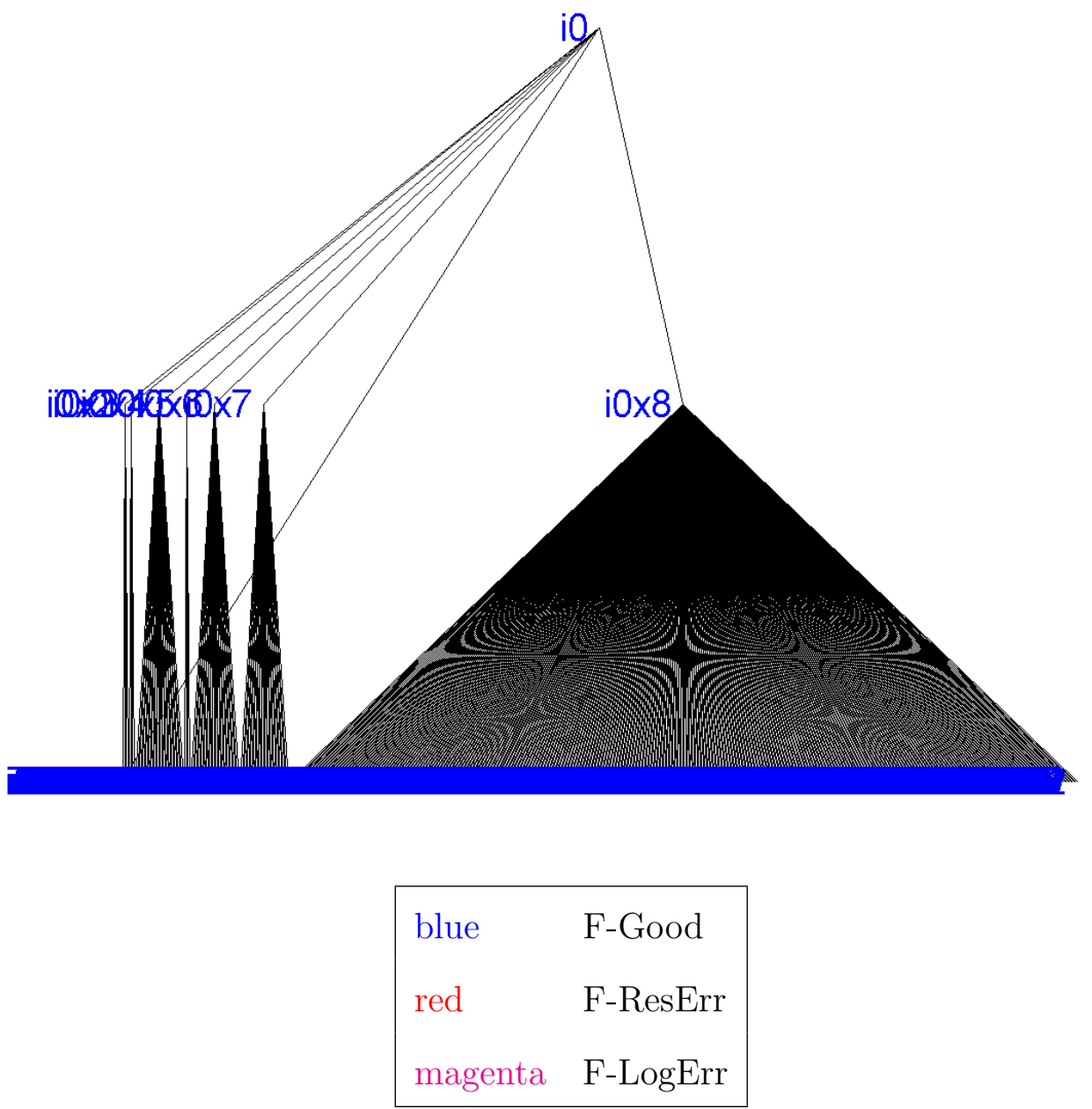

Figure 6.2: Tree graph of research strategy for initial phase. 


\subsection{Phase 1 - Initial Phase}

These are the intermediate knowledge state indexes of the strategy constructed in Phase 1:

\begin{tabular}{|l|l|}
\cline { 2 - 2 } \multicolumn{1}{l|}{} & $\mathrm{i} 0$ \\
\hline State and Type & F-Good intermediate index \\
\hline Samples Available & 90 \\
\hline Obtained Facts & $\begin{array}{l}\text { Potentially Significant Predictors: } \\
C S, F R, P A\end{array}$ \\
\hline Task List & screen $(B u r r S i z e(C S, F R, P A))$ \\
\hline Experimental Design & 3 variable 2-level full factorial, 3 reps \\
\hline Design Cost & 24 \\
\hline
\end{tabular}

Table 6.1: Descriptions of the knowledge state index i0 during Phase 1 


\subsection{Phase 1 - Initial Phase}

\begin{tabular}{|c|c|}
\hline & $\mathrm{i} 0 \mathrm{x} 2$ \\
\hline State and Type & F-Good intermediate index \\
\hline Samples Available & 66 \\
\hline Obtained Facts & $\begin{array}{l}\text { Potentially Significant Predictors: } \\
C S, F R, P A \\
\text { Screened Predictors: } C S\end{array}$ \\
\hline Task List & quadraticmodel(BurrSize $(C S))$ \\
\hline Experimental Design & $\begin{array}{l}1 \text { variable CCD, } 3 \text { axial reps, } 3 \text { factorial } \\
\text { reps, } 6 \text { center points }\end{array}$ \\
\hline Design Cost & 18 \\
\hline
\end{tabular}

Table 6.2: Descriptions of the knowledge state index i0x2 during Phase 1 


\subsection{Phase 1 - Initial Phase}

\begin{tabular}{|c|c|}
\hline & $\mathrm{i} 0 \times 3$ \\
\hline State and Type & F-Good intermediate index \\
\hline Samples Available & 66 \\
\hline Obtained Facts & $\begin{array}{l}\text { Potentially Significant Predictors: } \\
C S, F R, P A \\
\text { Screened Predictors: } F R\end{array}$ \\
\hline Task List & quadraticmodel $($ BurrSize $(F R))$ \\
\hline Experimental Design & $\begin{array}{l}1 \text { variable CCD, } 3 \text { axial reps, } 3 \text { factorial } \\
\text { reps, } 6 \text { center points }\end{array}$ \\
\hline Design Cost & 18 \\
\hline
\end{tabular}

Table 6.3: Descriptions of the knowledge state index i0x3 during Phase 1 


\subsection{Phase 1 - Initial Phase}

\begin{tabular}{|l|l|}
\cline { 2 - 2 } \multicolumn{1}{l|}{} & $\mathrm{i} 0 \mathrm{x} 4$ \\
\hline State and Type & F-Good intermediate index \\
\hline Samples Available & 66 \\
\hline Obtained Facts & $\begin{array}{l}\text { Potentially Significant Predictors: } \\
\text { Screened Predictors: } C S, F R\end{array}$ \\
\hline Task List & quadraticmodel(Burr $S i z e(C S, F R))$ \\
\hline Experimental Design & 2 variable CCD, 3 axial reps, 3 factorial \\
& reps, 8 center points \\
\hline Design Cost & 32 \\
\hline
\end{tabular}

Table 6.4: Descriptions of the knowledge state index i0x4 during Phase 1 


\subsection{Phase 1 - Initial Phase}

\begin{tabular}{|c|c|}
\hline & $\mathrm{i} 0 \times 5$ \\
\hline State and Type & F-Good intermediate index \\
\hline Samples Available & 66 \\
\hline Obtained Facts & $\begin{array}{l}\text { Potentially Significant Predictors: } \\
C S, F R, P A \\
\text { Screened Predictors: } P A\end{array}$ \\
\hline Task List & quadraticmodel $($ BurrSize $(P A))$ \\
\hline Experimental Design & $\begin{array}{l}1 \text { variable CCD, } 3 \text { axial reps, } 3 \text { factorial } \\
\text { reps, } 6 \text { center points }\end{array}$ \\
\hline Design Cost & 18 \\
\hline
\end{tabular}

Table 6.5: Descriptions of the knowledge state index i0x5 during Phase 1 


\subsection{Phase 1 - Initial Phase}

\begin{tabular}{|l|l|}
\cline { 2 - 2 } \multicolumn{1}{l|}{} & i0x6 \\
\hline State and Type & F-Good intermediate index \\
\hline Samples Available & 66 \\
\hline Obtained Facts & $\begin{array}{l}\text { Potentially Significant Predictors: } \\
\text { Screened Predictors: } C S, P A\end{array}$ \\
\hline Task List & quadraticmodel(Burr $S i z e(C S, P A))$ \\
\hline Experimental Design & 2 variable CCD, 3 axial reps, 3 factorial \\
& reps, 8 center points \\
\hline Design Cost & 32 \\
\hline
\end{tabular}

Table 6.6: Descriptions of the knowledge state index i0x6 during Phase 1 


\subsection{Phase 1 - Initial Phase}

\begin{tabular}{|l|l|}
\cline { 2 - 2 } \multicolumn{1}{l|}{} & $\mathrm{i} 0 \mathrm{x} 7$ \\
\hline State and Type & F-Good intermediate index \\
\hline Samples Available & 66 \\
\hline Obtained Facts & $\begin{array}{l}\text { Potentially Significant Predictors: } \\
\text { Screened Predictors: } F R, P A\end{array}$ \\
\hline Task List & quadraticmodel(Burr Size $(F R, P A))$ \\
\hline Experimental Design & 2 variable CCD, 3 axial reps, 3 factorial \\
& reps, 8 center points \\
\hline Design Cost & 32 \\
\hline
\end{tabular}

Table 6.7: Descriptions of the knowledge state index i0x7 during Phase 1 


\subsection{Phase 1 - Initial Phase}

\begin{tabular}{|l|l|}
\cline { 2 - 2 } \multicolumn{1}{l|}{} & $\mathrm{i} 0 \mathrm{x} 8$ \\
\hline State and Type & F-Good intermediate index \\
\hline Samples Available & 66 \\
\hline Obtained Facts & $\begin{array}{l}\text { Potentially Significant Predictors: } \\
C S, F, P A\end{array}$ \\
\hline Task List & quadraticmodel(Burr $S i z e(C S, F R, P A))$ \\
\hline Experimental Design & 3 variable CCD, 3 axial reps, 3 factorial \\
& reps, 10 center points \\
\hline Design Cost & 52 \\
\hline
\end{tabular}

Table 6.8: Descriptions of the knowledge state index i0x8 during Phase 1 


\subsection{Phase 1 - Initial Phase}

\subsubsection{Model Approximation Testing - Initial Phase}

\section{Phase 1 - Test 1}

Function to Approximate: BurrSize $=0.71 C S+0.31 F R+0.46 P A-0.01 C S *$ $C S-0.01 F R * F R-0.01 P A * P A+\epsilon, \epsilon \sim \mathrm{N}(0,0.01)$

The initial knowledge state index is i0, which is described in Table 6.9.

\begin{tabular}{|l|l|}
\cline { 2 - 2 } \multicolumn{1}{l|}{} & $\mathrm{i} 0$ \\
\hline State and Type & F-Good intermediate index \\
\hline Samples Available & 90 \\
\hline Obtained Facts & $\begin{array}{l}\text { Potentially Significant Predictors: } \\
C S, F R, P A\end{array}$ \\
\hline Task List & screen $(B$ Brr Size $(C S, F R, P A))$ \\
\hline Experimental Design & 3 variable 2-level full factorial, 3 reps \\
\hline Design Cost & 24 \\
\hline
\end{tabular}

Table 6.9: Descriptions of the knowledge state index during Move 1 of Phase 1 - Test 1

After performing the planned move of i0, the predictors that passed the screening process are CuttingSpeed, FeedRate, and PointAngle. As the result of the experimental events at the previous knowledge state index, the current knowledge state index is now $\mathrm{i} 0 \mathrm{x} 8$, which is described in Table 6.10. 


\subsection{Phase 1 - Initial Phase}

\begin{tabular}{|l|l|}
\cline { 2 - 2 } \multicolumn{1}{l|}{} & i0x8 \\
\hline State and Type & F-Good intermediate index \\
\hline Samples Available & 66 \\
\hline Obtained Facts & $\begin{array}{l}\text { Potentially Significant Predictors: } \\
C S, F R, P A\end{array}$ \\
\hline Task List & quadraticmodel $($ Burr $S i z e(C S, F R, P A))$ \\
\hline Experimental Design & 3 variable CCD, 3 axial reps, 3 factorial \\
& reps, 10 center points \\
\hline Design Cost & 52 \\
\hline
\end{tabular}

Table 6.10: Descriptions of the knowledge state index during Move 2 of Phase 1 - Test 1

After performing the planned move of $\mathrm{i} 0 \mathrm{x} 8$, the model is estimated to be Burr Size $=0.016+0.71 C S+0.31 F R+0.46 P A-0.01 C S * C S-9.9 \mathrm{e}-3 F R * F R-0.01 P A * P A$. As the result of the experimental events at the previous knowledge state index, the current knowledge state index is now i0x8x336, which is described in Table 6.11: 


\subsection{Phase 1 - Initial Phase}

\begin{tabular}{|l|l|}
\cline { 2 - 2 } \multicolumn{1}{l|}{} & $\mathrm{i} 0 \mathrm{x} 8 \mathrm{x} 336$ \\
\hline State and Type & F-Good terminating index \\
\hline Samples Available & 14 \\
\hline Obtained Facts & $\begin{array}{l}\text { Potentially Significant Predictors: } \\
C S, F R, P A \\
\text { Screened Predictors: } C S, F R, P A \\
\text { Modeled Predictors: } \\
C S, F R, P A, C S * C S, F R * F R, P A * P A\end{array}$ \\
\hline Task List & FinishGood \\
\hline Experimental Design & Finish \\
\hline Design Cost & 0 \\
\hline
\end{tabular}

Table 6.11: Descriptions of the knowledge state index during Move 3 of Phase 1 - Test 1

A response function has been successfully modeled, so experimentation can stop, and the final results can be shown. The type of termination, the main experimental results, and the overall cost are displayed in Figure 6.12. 


\subsection{Phase 1 - Initial Phase}

\begin{tabular}{|l|l|}
\cline { 2 - 2 } \multicolumn{1}{c|}{} & Results \\
\hline Terminating Index State & F-Good \\
\hline Experimental Results & Estimated Function: Burr Size $=0.016+$ \\
& $0.71 C S+0.31 F R+0.46 P A-0.01 C S * C S$ \\
& $-9.9 \mathrm{e}-3 F R * F R-0.01 P A * P A$ \\
\hline Data Source Function & BurrSize $=0.71 C S+0.31 F R+0.46 P A-$ \\
\hline Original Number of Samples & 90 \\
\hline Total Sample Cost & 76 \\
\hline Number Of Samples Left & 14 \\
\hline
\end{tabular}

Table 6.12: Final Results of Phase 1 - Test 1

Phase 1 - Test 2

Function to Approximate: BurrSize $=0.71 C S-0.31 F R+0.46 P A+\epsilon, \epsilon$ $\sim \mathrm{N}(0,0.01)$

The initial knowledge state index is i0, which is described in Table 6.13: 


\subsection{Phase 1 - Initial Phase}

\begin{tabular}{|l|l|}
\cline { 2 - 2 } \multicolumn{1}{l|}{} & i0 \\
\hline State and Type & F-Good intermediate index \\
\hline Samples Available & 90 \\
\hline Obtained Facts & $\begin{array}{l}\text { Potentially Significant Predictors: } \\
C S, F R, P A\end{array}$ \\
\hline Task List & screen $(B u r r S i z e(C S, F R, P A))$ \\
\hline Experimental Design & 3 variable 2-level full factorial, 3 reps \\
\hline Design Cost & 24 \\
\hline
\end{tabular}

Table 6.13: Descriptions of the knowledge state index during Move 1 of Phase 1 - Test 2

After performing the planned move of i0, the predictors that passed the screening process are CuttingSpeed, FeedRate, and PointAngle. As the result of the experimental events at the previous knowledge state index, the current knowledge state index is now i0x8, which is described in Table 6.14. 


\subsection{Phase 1 - Initial Phase}

\begin{tabular}{|l|l|}
\cline { 2 - 2 } \multicolumn{1}{l|}{} & i0x8 \\
\hline State and Type & F-Good intermediate index \\
\hline Samples Available & 66 \\
\hline Obtained Facts & $\begin{array}{l}\text { Potentially Significant Predictors: } \\
\text { Screened Predictors: } C S, F R, P A\end{array}$ \\
\hline Task List & quadraticmodel $(B u r r S i z e(C S, F R, P A))$ \\
\hline Experimental Design & 3 variable CCD, 3 axial reps, 3 factorial \\
& reps, 10 center points \\
\hline Design Cost & 52 \\
\hline
\end{tabular}

Table 6.14: Descriptions of the knowledge state index during Move 2 of Phase 1 - Test 2

After performing the planned move of i0x8, the model is estimated to be BurrSize $=0.11+0.71 C S-0.32 F R+0.46 P A+1.8 \mathrm{e}-5 P A * P A$. As the result of the experimental events at the previous knowledge state index, the current knowledge state index is now i0x8x264, which is described in Table 6.15: 


\subsection{Phase 1 - Initial Phase}

\begin{tabular}{|l|l|}
\cline { 2 - 2 } \multicolumn{1}{l|}{} & i0x8x264 \\
\hline State and Type & F-Good terminating index \\
\hline Samples Available & 14 \\
\hline Obtained Facts & $\begin{array}{l}\text { Potentially Significant Predictors: } \\
\text { Screened Predictors: } C S, F R, P A\end{array}$ \\
\hline Task List & \begin{tabular}{l} 
Modeled Predictors: $C S, F R, P A, P A * P A$ \\
\hline Experimental Design
\end{tabular} \\
\hline Design Cost & Finish \\
\hline
\end{tabular}

Table 6.15: Descriptions of the knowledge state index during Move 3 of Phase 1 - Test 2

A response function has been successfully modeled, so experimentation can stop, and the final results can be shown. The type of termination, the main experimental results, and the overall cost are displayed in Figure 6.16. 


\subsection{Phase 1 - Initial Phase}

\begin{tabular}{|l|l|}
\cline { 2 - 2 } \multicolumn{1}{c|}{} & Results \\
\hline Terminating Index State & F-Good \\
\hline Experimental Results & $\begin{array}{l}\text { Estimated Function: BurrSize }=0.11+ \\
0.71 C S-0.32 F R+0.46 P A+ \\
1.8 \mathrm{e}-5 P A * P A\end{array}$ \\
\hline Data Source Function & BurrSize $=0.71 C S-0.31 F R+0.46 P A+$ \\
\hline Original Number of Samples & 90 \\
\hline Total Sample Cost & 76 \\
\hline Number Of Samples Left & 14 \\
\hline
\end{tabular}

Table 6.16: Final Results of Phase 1 - Test 2

\section{Phase 1 - Test 3}

Function to Approximate: BurrSize $=-0.01 C S * C S+0.05 C S * F R-$ $0.01 F R * F R+\epsilon, \epsilon \sim \mathrm{N}(0,0.01)$

The initial knowledge state index is i0, which is described in Table 6.17; 


\subsection{Phase 1 - Initial Phase}

\begin{tabular}{|l|l|}
\cline { 2 - 2 } \multicolumn{1}{c|}{} & i0 \\
\hline State and Type & F-Good intermediate index \\
\hline Samples Available & 90 \\
\hline Obtained Facts & $\begin{array}{l}\text { Potentially Significant Predictors: } \\
C S, F R, P A\end{array}$ \\
\hline Task List & screen $(B u r r S i z e(C S, F R, P A))$ \\
\hline Experimental Design & 3 variable 2-level full factorial, 3 reps \\
\hline Design Cost & 24 \\
\hline
\end{tabular}

Table 6.17: Descriptions of the knowledge state index during Move 1 of Phase 1 - Test 3

After performing the planned move of i0, the predictors that passed the screening process are CuttingSpeed and FeedRate. As the result of the experimental events at the previous knowledge state index, the current knowledge state index is now i0x4, which is described in Table 6.18, 


\subsection{Phase 1 - Initial Phase}

\begin{tabular}{|l|l|}
\cline { 2 - 2 } \multicolumn{1}{l|}{} & $\mathrm{i} 0 \mathrm{x} 4$ \\
\hline State and Type & F-Good intermediate index \\
\hline Samples Available & 66 \\
\hline Obtained Facts & $\begin{array}{l}\text { Potentially Significant Predictors: } \\
\text { Screened Predictors: } C S, F R\end{array}$ \\
\hline Task List & quadraticmodel $($ Burr $S i z e(C S, F R))$ \\
\hline Experimental Design & 2 variable CCD, 3 axial reps, 3 factorial \\
& reps, 8 center points \\
\hline Design Cost & 32 \\
\hline
\end{tabular}

Table 6.18: Descriptions of the knowledge state index during Move 2 of Phase 1 - Test 3

After performing the planned move of $\mathrm{i} 0 \mathrm{x} 4$, the model is estimated to be BurrSize $=0.034-0.01 C S * C S+0.05 C S * F R-9.8 \mathrm{e}-3 F R * F R$. As the result of the experimental events at the previous knowledge state index, the current knowledge state index is now i0x4x29, which is described in Table 6.19. 


\subsection{Phase 1 - Initial Phase}

\begin{tabular}{|l|l|}
\cline { 2 - 2 } \multicolumn{1}{c|}{} & $\mathrm{i} 0 \mathrm{x} 4 \mathrm{x} 29$ \\
\hline State and Type & F-Good terminating index \\
\hline Samples Available & 34 \\
\hline Obtained Facts & $\begin{array}{l}\text { Potentially Significant Predictors: } \\
C S, F R, P A\end{array}$ \\
& $\begin{array}{l}\text { Screened Predictors: } C S, F R \\
\text { Modeled Predictors: }\end{array}$ \\
\hline Task List & $C S * C S, C S * F R, F R * F R$ \\
\hline Experimental Design & Finish $F$ Good \\
\hline Design Cost & 0 \\
\hline
\end{tabular}

Table 6.19: Descriptions of the knowledge state index during Move 3 of Phase 1 - Test 3

A response function has been successfully modeled, so experimentation can stop, and the final results can be shown. The type of termination, the main experimental results, and the overall cost are displayed in Figure 6.20. 
6.2 Phase 1 - Initial Phase

\begin{tabular}{|l|l|}
\cline { 2 - 2 } \multicolumn{1}{c|}{} & Results \\
\hline Terminating Index State & F-Good \\
\hline Experimental Results & $\begin{array}{l}\text { Estimated Function: BurrSize }=0.034- \\
0.01 C S * C S+0.05 C S * F R- \\
9.8 \mathrm{e}-3 F R * F R\end{array}$ \\
\hline Data Source Function & BurrSize $=-0.01 C S * C S+0.05 C S * F R$ \\
\hline Original Number of Samples & 90 \\
\hline Total Sample Cost & 56 \\
\hline Number Of Samples Left & 34 \\
\hline
\end{tabular}

Table 6.20: Final Results of Phase 1 - Test 3

\section{Phase 1 - Test 4}

Function to Approximate: BurrSize $=\epsilon, \epsilon \sim \mathrm{N}(0,0.01)$

The initial knowledge state index is i0, which is described in Table 6.21: 


\subsection{Phase 1 - Initial Phase}

\begin{tabular}{|l|l|}
\cline { 2 - 2 } \multicolumn{1}{l|}{} & i0 \\
\hline State and Type & F-Good intermediate index \\
\hline Samples Available & 90 \\
\hline Obtained Facts & $\begin{array}{l}\text { Potentially Significant Predictors: } \\
C S, F R, P A\end{array}$ \\
\hline Task List & screen $(B u r r S i z e(C S, F R, P A))$ \\
\hline Experimental Design & 3 variable 2-level full factorial, 3 reps \\
\hline Design Cost & 24 \\
\hline
\end{tabular}

Table 6.21: Descriptions of the knowledge state index during Move 1 of Phase 1 - Test 4

After performing the planned move of i0, no variables passed the screening process. As the result of the experimental events at the previous knowledge state index, the current knowledge state index is now i0x1, which is described in Table 6.22; 


\subsection{Phase 1 - Initial Phase}

\begin{tabular}{|l|l|}
\cline { 2 - 2 } \multicolumn{1}{c|}{} & i0x1 \\
\hline State and Type & F-Good terminating index \\
\hline Samples Available & 66 \\
\hline Obtained Facts & $\begin{array}{l}\text { Potentially Significant Predictors: } \\
\text { Screened Vars: None }\end{array}$ \\
\hline Task List & FinishGood \\
\hline Experimental Design & Finish \\
\hline Design Cost & 0 \\
\hline
\end{tabular}

Table 6.22: Descriptions of the knowledge state index during Move 2 of Phase 1 - Test 4

A response function has been successfully modeled, so experimentation can stop, and the final results can be shown. The type of termination, the main experimental results, and the overall cost are displayed in Figure 6.23. 


\subsection{Phase 1 - Initial Phase}

\begin{tabular}{|l|l|}
\cline { 2 - 2 } \multicolumn{1}{c|}{} & Results \\
\hline Terminating Index State & F-Good \\
\hline Experimental Results & Screened predictors of BurrSize: None \\
\hline Data Source Function & BurrSize $=\epsilon, \epsilon \sim \mathrm{N}(0,0.01)$ \\
\hline Original Number of Samples & 90 \\
\hline Total Sample Cost & 24 \\
\hline Number Of Samples Left & 66 \\
\hline
\end{tabular}

Table 6.23: Final Results of Phase 1 - Test 4

\section{Phase 1 Summary:}

The research strategy is F-Good, and the sponsor is satisfied with the quality of the model approximations. 


\subsection{Phase 2 - Minimal Strategy Phase}

\subsubsection{Preliminary Sponsor-Designer Discussions}

The sponsor wants to know what is the lowest amount of samples that produces an F-Good research strategy using the same preferences as Example 1, and wants to know how well that strategy performs.

\subsubsection{Specifications Expressed Formally by Designer for Phase 2}

Number of samples: minimal number required for F-Good initial knowledge state index

\section{Project Satisfaction Condition:}

All predictors screened out, or response function constructed

Task Specification Function:

If potential predictors have not gone through screening (in order of preference, greatest to least):

screen(BurrSize $(C S, F R, P A))$

screen(BurrSize $(C S, F R))$ 


\subsection{Phase 2 - Minimal Strategy Phase}

screen(BurrSize $(C S))$

If potential predictors have gone through screening (in order of preference, greatest to least):

quadraticmodel(BurrSize(screened predictors)), linearmodel(BurrSize(screened predictors)), linearmodel(BurrSize(screened predictors - screened predictor of least priority))

linearmodel(BurrSize(highest priority screened variable)),

\section{Experimental Design Preference Ordering Function:}

For screening (in order of preference, greatest to least):

Full Factorial Design, 3 replications

Full Factorial Design, 2 replications

For linear modeling (in order of preference, greatest to least):

Full Factorial Design, 3 replications

Full Factorial Design, 2 replications

For quadratic modeling:

Central Composite Design 


\subsection{Phase 2 - Minimal Strategy Phase}

maximum number of center points $=($ number of screened predictors +2$) * 2$

minimum number of center points $=$ number of screened predictors +4

maximum number of factorial points $=3$

minimum number of factorial points $=2$

maximum number of axial points $=3$

minimum number of axial points $=2$

start with maximum values, reduce number of center points first, then number of axial points, then number of factorial points 


\subsection{Phase 2 - Minimal Strategy Phase}

\subsubsection{Model Approximation Testing - Minimal Strategy Phase}

By binary search between 0 and 90, the lowest number of samples required to construct an F-Good research strategy under the conditions of Section 6.3.2 is determined to be 8 . Figure 6.3 is a visual representation of the F-Good research strategy, determined from Section 6.3.2:

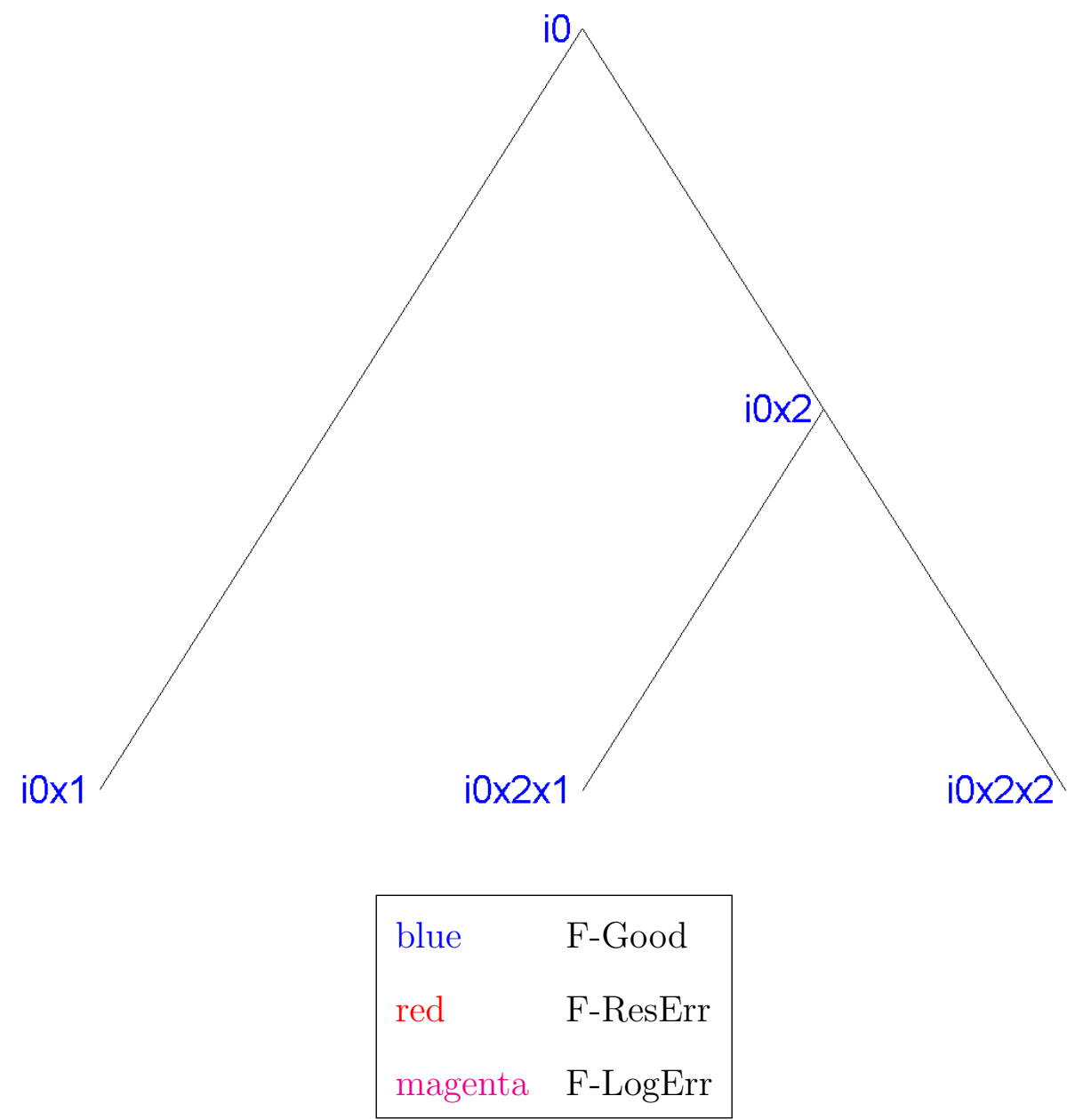

Figure 6.3: Tree graph of research strategy for minimal strategy phase. 


\subsection{Phase 2 - Minimal Strategy Phase}

These are the intermediate knowledge state indexes of the strategy constructed in Phase 2:

\begin{tabular}{|l|l|}
\cline { 2 - 2 } \multicolumn{1}{l|}{} & i0 \\
\hline State and Type & F-Good intermediate index \\
\hline Samples Available & 8 \\
\hline Obtained Facts & $\begin{array}{l}\text { Potentially Significant Predictors: } \\
C S, F R, P A\end{array}$ \\
\hline Task List & screen $(B u r r S i z e(C S))$ \\
\hline Experimental Design & 1 variable 2-level full factorial, 2 reps \\
\hline Design Cost & 4 \\
\hline
\end{tabular}

Table 6.24: Descriptions of the knowledge state index i0 during Phase 2 


\subsection{Phase 2 - Minimal Strategy Phase}

\begin{tabular}{|l|l|}
\cline { 2 - 2 } \multicolumn{1}{l|}{} & i0x2 \\
\hline State and Type & F-Good intermediate index \\
\hline Samples Available & 4 \\
\hline Obtained Facts & $\begin{array}{l}\text { Potentially Significant Predictors: } \\
\text { CS } R, P A\end{array}$ \\
\hline Task List & linearmodel(BurrSize $(C S))$ \\
\hline Experimental Design & 1 variable 2-level full factorial, 2 reps \\
\hline Design Cost & 4 \\
\hline
\end{tabular}

Table 6.25: Descriptions of the knowledge state index i0x2 during Phase 2 


\subsubsection{Model Approximation Testing - Minimal Strategy Phase}

\section{Phase 2 - Test 1}

Function to Approximate: BurrSize $=0.71 C S+0.31 F R+0.46 P A-0.01 C S *$ $C S-0.01 F R * F R-0.01 P A * P A+\epsilon, \epsilon \sim \mathrm{N}(0,0.01)$

The initial knowledge state index is i0, which is described in Table 6.26:

\begin{tabular}{|l|l|}
\cline { 2 - 2 } \multicolumn{1}{c|}{} & i0 \\
\hline State and Type & F-Good intermediate index \\
\hline Samples Available & 8 \\
\hline Obtained Facts & $\begin{array}{l}\text { Potentially Significant Predictors: } \\
C S, F R, P A\end{array}$ \\
\hline Task List & screen $(B u r r S i z e(C S))$ \\
\hline Experimental Design & 1 variable 2-level full factorial, 2 reps \\
\hline Design Cost & 4 \\
\hline
\end{tabular}

Table 6.26: Descriptions of the knowledge state index during Move 1 of Phase 2 - Test 1

After performing the planned move of i0, the predictor that passed the screening process is CuttingSpeed. As the result of the experimental events at the previous knowledge state index, the current knowledge state index is now i0x2, which is described in Table 6.27, 


\subsection{Phase 2 - Minimal Strategy Phase}

\begin{tabular}{|l|l|}
\cline { 2 - 2 } \multicolumn{1}{l|}{} & i0x2 \\
\hline State and Type & F-Good intermediate index \\
\hline Samples Available & 4 \\
\hline Obtained Facts & $\begin{array}{l}\text { Potentially Significant Predictors: } \\
\text { Screened Predictors: } C S\end{array}$ \\
\hline Task List & linearmodel $($ Burr Size $(C S))$ \\
\hline Experimental Design & 1 variable 2-level full factorial, 2 reps \\
\hline Design Cost & 4 \\
\hline
\end{tabular}

Table 6.27: Descriptions of the knowledge state index during Move 2 of Phase 2 - Test 1

After performing the planned move of i0x2, the model is estimated to be BurrSize $=18.0+0.01 C S$. As the result of the experimental events at the previous knowledge state index, the current knowledge state index is now $\mathrm{i} 0 \mathrm{x} 2 \mathrm{x} 2$, which is described in Table 6.28: 


\subsection{Phase 2 - Minimal Strategy Phase}

\begin{tabular}{|c|c|}
\hline & $\mathrm{i} 0 \times 2 \times 2$ \\
\hline State and Type & F-Good terminating index \\
\hline Samples Available & 0 \\
\hline Obtained Facts & $\begin{array}{l}\text { Potentially Significant Predictors: } \\
C S, F R, P A \\
\text { Screened Predictors: } C S \\
\text { Modeled Predictors: } C S\end{array}$ \\
\hline Task List & FinishGood \\
\hline Experimental Design & Finish \\
\hline Design Cost & 0 \\
\hline
\end{tabular}

Table 6.28: Descriptions of the knowledge state index during Move 3 of Phase 2 - Test 1

A response function has been successfully modeled, so experimentation can stop, and the final results can be shown. The type of termination, the main experimental results, and the overall cost are displayed in Figure 6.29. 


\subsection{Phase 2 - Minimal Strategy Phase}

\begin{tabular}{|l|l|}
\cline { 2 - 2 } \multicolumn{1}{c|}{} & Results \\
\hline Terminating Index State & F-Good \\
\hline Experimental Results & $\begin{array}{l}\text { Estimated Function: BurrSize }=18.0+ \\
0.01 C S\end{array}$ \\
\hline Data Source Function & $\begin{array}{l}\text { BurrSize }=0.71 C S+0.31 F R+0.46 P A-C S-0.01 F R * F R-0.01 P A * P A \\
0.01 C S *-01)\end{array}$ \\
\hline Original Number of Samples & 8 \\
\hline Total Sample Cost & 8 \\
\hline Number Of Samples Left & 0 \\
\hline
\end{tabular}

Table 6.29: Final Results of Phase 2 - Test 1

\section{Phase 2 - Test 2}

Function to Approximate: BurrSize $=0.71 C S-0.31 F R+0.46 P A+\epsilon, \epsilon$ $\sim \mathrm{N}(0,0.01)$

The predictor that passed the screening process is CuttingSpeed. The model is estimated to be BurrSize $=5.7+0.71 C S$. The terminating knowledge state index is described in Table 6.30: 


\subsection{Phase 2 - Minimal Strategy Phase}

\begin{tabular}{|l|l|}
\cline { 2 - 2 } \multicolumn{1}{l|}{} & $\mathrm{i} 0 \mathrm{x} 2 \mathrm{x} 2$ \\
\hline State and Type & F-Good terminating index \\
\hline Samples Available & 0 \\
\hline Obtained Facts & $\begin{array}{l}\text { Potentially Significant Predictors: } \\
\text { Screened Predictors: } C S\end{array}$ \\
\hline Task List & \begin{tabular}{l} 
Modeled Predictors: $C S$ \\
\hline Experimental Design
\end{tabular} \\
\hline Design Cost & Finish \\
\hline
\end{tabular}

Table 6.30: Descriptions of the knowledge state index during Move 1 of Phase 2 - Test 2

A response function has been successfully modeled, so experimentation can stop, and the final results can be shown. The type of termination, the main experimental results, and the overall cost are displayed in Figure 6.31. 


\subsection{Phase 2 - Minimal Strategy Phase}

\begin{tabular}{|l|l|}
\cline { 2 - 2 } \multicolumn{1}{c|}{} & Results \\
\hline Terminating Index State & F-Good \\
\hline Experimental Results & $\begin{array}{l}\text { Estimated Function: BurrSize }=5.7+ \\
0.71 C S\end{array}$ \\
\hline Data Source Function & BurrSize $=0.71 C S-0.31 F R+0.46 P A+$ \\
\hline Original Number of Samples & 8 \\
\hline Total Sample Cost & 8 \\
\hline Number Of Samples Left & 0 \\
\hline
\end{tabular}

Table 6.31: Final Results of Phase 2 - Test 2

\section{Phase 2 - Test 3}

Function to Approximate: BurrSize $=-0.01 C S * C S+0.05 C S * F R$ $0.01 F R * F R+\epsilon, \epsilon \sim \mathrm{N}(0,0.01)$

The predictor that passed the screening process is CuttingSpeed. The model is estimated to be BurrSize $=7.8+0.05 C$ S . The terminating knowledge state index is described in Table 6.32: 


\subsection{Phase 2 - Minimal Strategy Phase}

\begin{tabular}{|l|l|}
\cline { 2 - 2 } \multicolumn{1}{l|}{} & $\mathrm{i} 0 \mathrm{x} 2 \mathrm{x} 2$ \\
\hline State and Type & F-Good terminating index \\
\hline Samples Available & 0 \\
\hline Obtained Facts & $\begin{array}{l}\text { Potentially Significant Predictors: } \\
\text { Screened Predictors: } C S\end{array}$ \\
\hline Task List & \begin{tabular}{l} 
Modeled Predictors: $C S$ \\
\hline Experimental Design
\end{tabular} \\
\hline Design Cost & Finish \\
\hline
\end{tabular}

Table 6.32: Descriptions of the knowledge state index during Move 1 of Phase 2 - Test 3

A response function has been successfully modeled, so experimentation can stop, and the final results can be shown. The type of termination, the main experimental results, and the overall cost are displayed in Figure 6.33. 


\subsection{Phase 2 - Minimal Strategy Phase}

\begin{tabular}{|l|l|}
\cline { 2 - 2 } \multicolumn{1}{c|}{} & Results \\
\hline Terminating Index State & F-Good \\
\hline Experimental Results & $\begin{array}{l}\text { Estimated Function: BurrSize }=7.8+ \\
0.05 C S\end{array}$ \\
\hline Data Source Function & $\begin{array}{l}\text { BurrSize }=-0.01 C S * C S+0.05 C S * F R \\
-0.01 F R * F R+\epsilon, \epsilon \sim \mathrm{N}(0,0.01)\end{array}$ \\
\hline Original Number of Samples & 8 \\
\hline Total Sample Cost & 8 \\
\hline Number Of Samples Left & 0 \\
\hline
\end{tabular}

Table 6.33: Final Results of Phase 2 - Test 3

\section{Phase 2 - Test 4}

Function to Approximate: BurrSize $=\epsilon, \epsilon \sim \mathrm{N}(0,0.01)$

No predictors passed the screening process. The terminating knowledge state index is described in Table 6.34: 


\subsection{Phase 2 - Minimal Strategy Phase}

\begin{tabular}{|l|l|}
\cline { 2 - 2 } \multicolumn{1}{c|}{} & i0x1 \\
\hline State and Type & F-Good terminating index \\
\hline Samples Available & 4 \\
\hline Obtained Facts & $\begin{array}{l}\text { Potentially Significant Predictors: } \\
\text { Screened Vars: None }\end{array}$ \\
\hline Task List & FinishGood \\
\hline Experimental Design & Finish \\
\hline Design Cost & 0 \\
\hline
\end{tabular}

Table 6.34: Descriptions of the knowledge state index during Move 1 of Phase 2 - Test 4

A response function has been successfully modeled, so experimentation can stop, and the final results can be shown. The type of termination, the main experimental results, and the overall cost are displayed in Figure 6.35. 


\subsection{Phase 2 - Minimal Strategy Phase}

\begin{tabular}{|l|l|}
\cline { 2 - 2 } \multicolumn{1}{c|}{} & Results \\
\hline Terminating Index State & F-Good \\
\hline Experimental Results & Screened predictors of BurrSize: None \\
\hline Data Source Function & BurrSize $=\epsilon, \epsilon \sim \mathrm{N}(0,0.01)$ \\
\hline Original Number of Samples & 8 \\
\hline Total Sample Cost & 4 \\
\hline Number Of Samples Left & 4 \\
\hline
\end{tabular}

Table 6.35: Final Results of Phase 2 - Test 4

\section{Phase 2 Summary:}

While the constructed research strategy is F-Good and is considerably cheaper than the last research strategy, it is not nearly as able to perform model approximation. The sponsor does not like this particular research strategy, and wants to see if a minimal research strategy can be constructed that still performs the desired analytical tasks. 


\subsection{Phase 3 - No Task Compromise Minimal Strat- egy Phase}

\subsubsection{Preliminary Sponsor-Designer Discussions}

The sponsor didn't like the results of Example 2, and wants to know what is the lowest amount of samples that produces an F-Good research strategy using the same preferences as the previous example, except that for eliminating compromise for the task list, and wants to know how well that strategy performs.

\subsubsection{Specifications Expressed Formally by Designer for Phase 3}

Number of samples: minimal number required for F-Good initial knowledge state index

Project Satisfaction Condition:

All predictors screened out, or response function constructed

\section{Task Specification Function:}

If potential predictors have not gone through screening:

screen $(B u r r S i z e(C S, F R, P A))$ 


\subsection{Phase 3 - No Task Compromise Minimal Strategy Phase}

If potential predictors have gone through screening (in order of preference, greatest to least):

quadraticmodel(BurrSize(screened predictors))

\section{Experimental Design Preference Ordering Function:}

For screening (in order of preference, greatest to least):

Full Factorial Design, 3 replications

Full Factorial Design, 2 replications

For quadratic modeling:

Central Composite Design

maximum number of center points $=($ number of screened predictors +2$) * 2$

minimum number of center points $=$ number of screened predictors +4

maximum number of factorial points $=3$

minimum number of factorial points $=2$

maximum number of axial points $=3$

minimum number of axial points $=2$

start with maximum values, reduce number of center points first, then number of axial points, then number of factorial points 


\subsection{Phase 3 - No Task Compromise Minimal Strategy Phase}

\subsubsection{Constructed Research Strategy - No Task Compromise Minimal Strategy Phase}

By binary search between 0 and 90, the lowest number of samples required to

construct an F-Good research strategy under the conditions of Section 6.4.2 is determined to be 51. Figure 6.4 is a visual representation of the F-Good research strategy, determined from Section 6.4.2. 


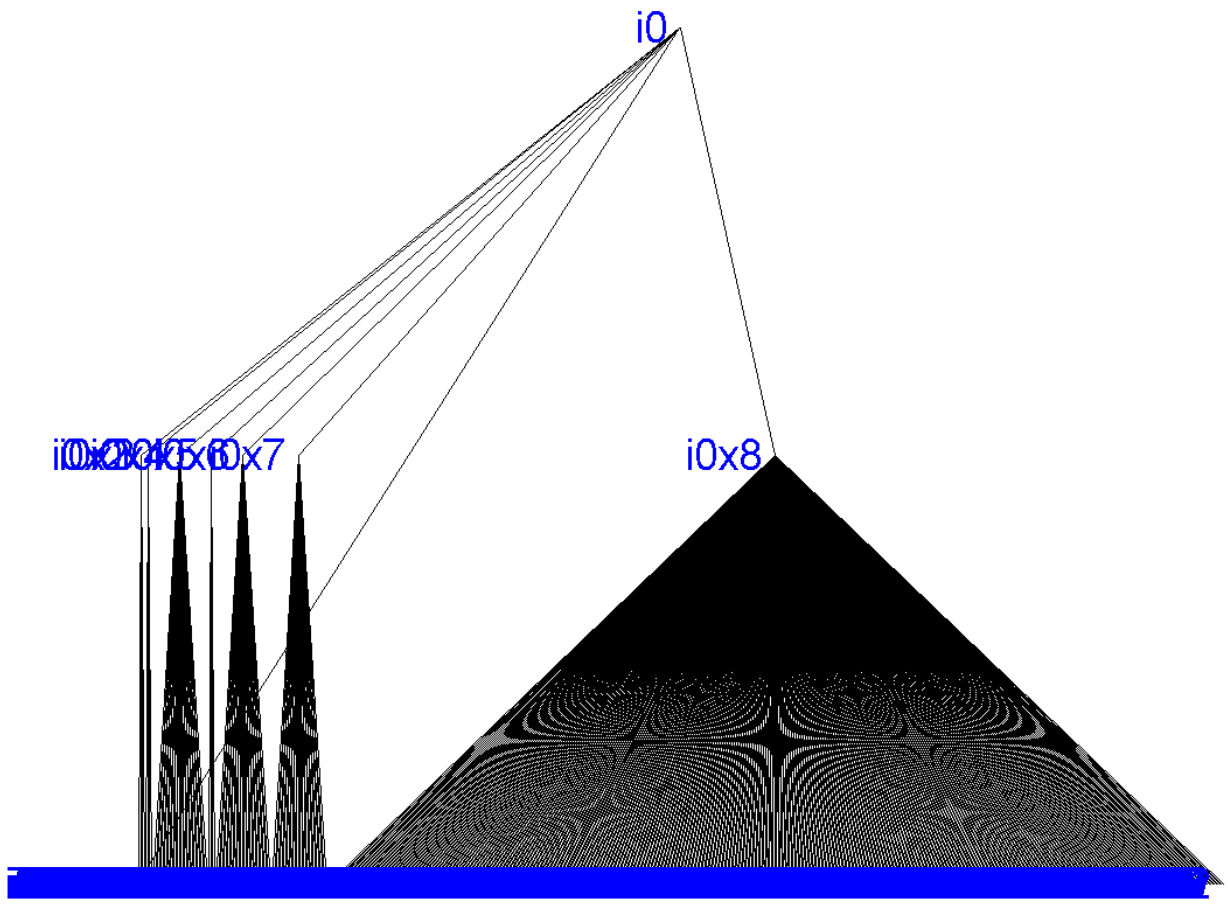

\begin{tabular}{|ll|}
\hline blue & F-Good \\
red & F-ResErr \\
magenta & F-LogErr \\
\hline
\end{tabular}

Figure 6.4: Tree graph of research strategy for no task compromise minimal strategy phase. 


\subsection{Phase 3 - No Task Compromise Minimal Strategy Phase}

These are the intermediate knowledge state indexes of the strategy constructed in Phase 3:

\begin{tabular}{|l|l|}
\cline { 2 - 2 } \multicolumn{1}{l|}{} & i0 \\
\hline State and Type & F-Good intermediate index \\
\hline Samples Available & 51 \\
\hline Obtained Facts & $\begin{array}{l}\text { Potentially Significant Predictors: } \\
C S, F R, P A\end{array}$ \\
\hline Task List & screen $(B u r r S i z e(C S, F R, P A))$ \\
\hline Experimental Design & 3 variable 2-level full factorial, 2 reps \\
\hline Design Cost & 16 \\
\hline
\end{tabular}

Table 6.36: Descriptions of the knowledge state index i0 during Phase 3 


\subsection{Phase 3 - No Task Compromise Minimal Strategy Phase}

\begin{tabular}{|c|c|}
\hline & $\mathrm{i} 0 \mathrm{x} 2$ \\
\hline State and Type & F-Good intermediate index \\
\hline Samples Available & 35 \\
\hline Obtained Facts & $\begin{array}{l}\text { Potentially Significant Predictors: } \\
C S, F R, P A \\
\text { Screened Predictors: } C S\end{array}$ \\
\hline Task List & quadraticmodel(BurrSize $(C S))$ \\
\hline Experimental Design & $\begin{array}{l}1 \text { variable CCD, } 3 \text { axial reps, } 3 \text { factorial } \\
\text { reps, } 6 \text { center points }\end{array}$ \\
\hline Design Cost & 18 \\
\hline
\end{tabular}

Table 6.37: Descriptions of the knowledge state index i0x2 during Phase 3 


\subsection{Phase 3 - No Task Compromise Minimal Strategy Phase}

\begin{tabular}{|c|c|}
\hline & $\mathrm{i} 0 \times 3$ \\
\hline State and Type & F-Good intermediate index \\
\hline Samples Available & 35 \\
\hline Obtained Facts & $\begin{array}{l}\text { Potentially Significant Predictors: } \\
C S, F R, P A \\
\text { Screened Predictors: } F R\end{array}$ \\
\hline Task List & quadraticmodel $($ BurrSize $(F R))$ \\
\hline Experimental Design & $\begin{array}{l}1 \text { variable CCD, } 3 \text { axial reps, } 3 \text { factorial } \\
\text { reps, } 6 \text { center points }\end{array}$ \\
\hline Design Cost & 18 \\
\hline
\end{tabular}

Table 6.38: Descriptions of the knowledge state index i0x3 during Phase 3 


\subsection{Phase 3 - No Task Compromise Minimal Strategy Phase}

\begin{tabular}{|l|l|}
\cline { 2 - 2 } \multicolumn{1}{l|}{} & $\mathrm{i} 0 \mathrm{x} 4$ \\
\hline State and Type & F-Good intermediate index \\
\hline Samples Available & 35 \\
\hline Obtained Facts & $\begin{array}{l}\text { Potentially Significant Predictors: } \\
\text { Screened Predictors: } C S, F R\end{array}$ \\
\hline Task List & quadraticmodel(Burr $S i z e(C S, F R))$ \\
\hline Experimental Design & 2 variable CCD, 3 axial reps, 3 factorial \\
& reps, 8 center points \\
\hline Design Cost & 32 \\
\hline
\end{tabular}

Table 6.39: Descriptions of the knowledge state index i0x4 during Phase 3 


\subsection{Phase 3 - No Task Compromise Minimal Strategy Phase}

\begin{tabular}{|c|c|}
\hline & $\mathrm{i} 0 \times 5$ \\
\hline State and Type & F-Good intermediate index \\
\hline Samples Available & 35 \\
\hline Obtained Facts & $\begin{array}{l}\text { Potentially Significant Predictors: } \\
C S, F R, P A \\
\text { Screened Predictors: } P A\end{array}$ \\
\hline Task List & quadraticmodel $($ BurrSize $(P A))$ \\
\hline Experimental Design & $\begin{array}{l}1 \text { variable CCD, } 3 \text { axial reps, } 3 \text { factorial } \\
\text { reps, } 6 \text { center points }\end{array}$ \\
\hline Design Cost & 18 \\
\hline
\end{tabular}

Table 6.40: Descriptions of the knowledge state index i0x5 during Phase 3 


\subsection{Phase 3 - No Task Compromise Minimal Strategy Phase}

\begin{tabular}{|l|l|}
\cline { 2 - 2 } \multicolumn{1}{l|}{} & i0x6 \\
\hline State and Type & F-Good intermediate index \\
\hline Samples Available & 35 \\
\hline Obtained Facts & $\begin{array}{l}\text { Potentially Significant Predictors: } \\
\text { Screened Predictors: } C S, P A\end{array}$ \\
\hline Task List & quadraticmodel(Burr Size $(C S, P A))$ \\
\hline Experimental Design & 2 variable CCD, 3 axial reps, 3 factorial \\
& reps, 8 center points \\
\hline Design Cost & 32 \\
\hline
\end{tabular}

Table 6.41: Descriptions of the knowledge state index i0x6 during Phase 3 


\subsection{Phase 3 - No Task Compromise Minimal Strategy Phase}

\begin{tabular}{|l|l|}
\cline { 2 - 2 } \multicolumn{1}{l|}{} & $\mathrm{i} 0 \mathrm{x} 7$ \\
\hline State and Type & F-Good intermediate index \\
\hline Samples Available & 35 \\
\hline Obtained Facts & $\begin{array}{l}\text { Potentially Significant Predictors: } \\
\text { Screened Predictors: } F R, P A\end{array}$ \\
\hline Task List & quadraticmodel(Burr Size $(F R, P A))$ \\
\hline Experimental Design & 2 variable CCD, 3 axial reps, 3 factorial \\
& reps, 8 center points \\
\hline Design Cost & 32 \\
\hline
\end{tabular}

Table 6.42: Descriptions of the knowledge state index i0x7 during Phase 3 


\subsection{Phase 3 - No Task Compromise Minimal Strategy Phase}

\begin{tabular}{|l|l|}
\cline { 2 - 2 } \multicolumn{1}{l|}{} & i0x8 \\
\hline State and Type & F-Good intermediate index \\
\hline Samples Available & 35 \\
\hline Obtained Facts & $\begin{array}{l}\text { Potentially Significant Predictors: } \\
\text { Screened Predictors: } C S, F R, P A\end{array}$ \\
\hline Task List & quadraticmodel(Burr $S i z e(C S, F R, P A))$ \\
\hline Experimental Design & 3 variable CCD, 2 axial reps, 2 factorial \\
& reps, 7 center points \\
\hline Design Cost & 35 \\
\hline
\end{tabular}

Table 6.43: Descriptions of the knowledge state index i0x8 during Phase 3 


\subsection{Phase 3 - No Task Compromise Minimal Strategy Phase}

\subsubsection{Model Approximation Testing - No Task Compromise Minimal Strategy Phase}

\section{Phase 3 - Test 1}

Function to Approximate: BurrSize $=0.71 C S+0.31 F R+0.46 P A-0.01 C S *$ $C S-0.01 F R * F R-0.01 P A * P A+\epsilon, \epsilon \sim \mathrm{N}(0,0.01)$

The initial knowledge state index is i0, which is described in Table 6.44:

\begin{tabular}{|l|l|}
\cline { 2 - 2 } \multicolumn{1}{c|}{} & i0 \\
\hline State and Type & F-Good intermediate index \\
\hline Samples Available & 51 \\
\hline Obtained Facts & $\begin{array}{l}\text { Potentially Significant Predictors: } \\
C S, F R, P A\end{array}$ \\
\hline Task List & screen $(B u r r S i z e(C S, F R, P A))$ \\
\hline Experimental Design & 3 variable 2-level full factorial, 2 reps \\
\hline Design Cost & 16 \\
\hline
\end{tabular}

Table 6.44: Descriptions of the knowledge state index during Move 1 of Phase 3 - Test 1

After performing the planned move of i0, the predictors that passed the screening process are CuttingSpeed, FeedRate, and PointAngle. As the result of the experimental events at the previous knowledge state index, the current knowledge state index is now i0x8, which is described in Table 6.45. 


\subsection{Phase 3 - No Task Compromise Minimal Strategy Phase}

\begin{tabular}{|l|l|}
\cline { 2 - 2 } \multicolumn{1}{l|}{} & i0x8 \\
\hline State and Type & F-Good intermediate index \\
\hline Samples Available & 35 \\
\hline Obtained Facts & $\begin{array}{l}\text { Potentially Significant Predictors: } \\
\text { Screened Predictors: } C S, F R, P A\end{array}$ \\
\hline Task List & quadraticmodel $(B u r r S i z e(C S, F R, P A))$ \\
\hline Experimental Design & 3 variable CCD, 2 axial reps, 2 factorial \\
& reps, 7 center points \\
\hline Design Cost & 35 \\
\hline
\end{tabular}

Table 6.45: Descriptions of the knowledge state index during Move 2 of Phase 3 - Test 1

After performing the planned move of i0x8, the model is estimated to be BurrSize $=-0.011+0.71 C S+0.31 F R+0.46 P A-0.01 C S * C S-0.01 F R * F R-0.01 P A * P A$. As the result of the experimental events at the previous knowledge state index, the current knowledge state index is now i0x8x336, which is described in Table 6.46: 


\subsection{Phase 3 - No Task Compromise Minimal Strategy Phase}

\begin{tabular}{|l|l|}
\cline { 2 - 2 } \multicolumn{1}{l|}{} & i0x8x336 \\
\hline State and Type & F-Good terminating index \\
\hline Samples Available & 0 \\
\hline & $\begin{array}{l}\text { Potentially Significant Predictors: } \\
C S, F R, P A\end{array}$ \\
Obtained Facts & $\begin{array}{l}\text { Screened Predictors: } C S, F R, P A \\
\text { Modeled Predictors: } \\
C S, F R, P A, C S * C S, F R * F R, P A * P A\end{array}$ \\
\hline Task List & FinishGood \\
\hline Experimental Design & Finish \\
\hline Design Cost & 0 \\
\hline
\end{tabular}

Table 6.46: Descriptions of the knowledge state index during Move 3 of Phase 3 - Test 1

A response function has been successfully modeled, so experimentation can stop, and the final results can be shown. The type of termination, the main experimental results, and the overall cost are displayed in Figure 6.47. 


\subsection{Phase 3 - No Task Compromise Minimal Strategy Phase}

\begin{tabular}{|l|l|}
\cline { 2 - 2 } \multicolumn{1}{c|}{} & Results \\
\hline Terminating Index State & F-Good \\
\hline Experimental Results & Estimated Function: BurrSize $=0.011+$ \\
& $0.71 C S+0.31 F R+0.46 P A-0.01 C S * C S$ \\
& $-0.01 F R * F R-0.01 P A * P A$ \\
\hline Data Source Function & BurrSize $=0.71 C S+0.31 F R+0.46 P A-$ \\
\hline Original Number of Samples & 51 \\
\hline Total Sample Cost & 51 \\
\hline Number Of Samples Left & 0 \\
\hline
\end{tabular}

Table 6.47: Final Results of Phase 3 - Test 1

\section{Phase 3 - Test 2}

Function to Approximate: BurrSize $=0.71 C S-0.31 F R+0.46 P A+\epsilon, \epsilon$ $\sim \mathrm{N}(0,0.01)$

The predictors that passed the screening process are CuttingSpeed, FeedRate, and PointAngle. The model is estimated to be BurrSize $=0.061+0.71 C S-0.31 F R$ $+0.46 P A$. The terminating knowledge state index is described in Table 6.48; 


\subsection{Phase 3 - No Task Compromise Minimal Strategy Phase}

\begin{tabular}{|l|l|}
\cline { 2 - 2 } \multicolumn{1}{l|}{} & $\mathrm{i} 0 \mathrm{x} 8 \mathrm{x} 8$ \\
\hline State and Type & F-Good terminating index \\
\hline Samples Available & 0 \\
\hline Obtained Facts & $\begin{array}{l}\text { Potentially Significant Predictors: } \\
\text { Screened Predictors: } C S, F R, P A\end{array}$ \\
\hline Task List & \begin{tabular}{l} 
Modeled Predictors: $C S, F R, P A$ \\
\hline Experimental Design
\end{tabular} \\
\hline Design Cost & Finish \\
\hline
\end{tabular}

Table 6.48: Descriptions of the knowledge state index during Move 1 of Phase 3 - Test 2

A response function has been successfully modeled, so experimentation can stop, and the final results can be shown. The type of termination, the main experimental results, and the overall cost are displayed in Figure 6.49. 


\subsection{Phase 3 - No Task Compromise Minimal Strategy Phase}

\begin{tabular}{|l|l|}
\cline { 2 - 2 } \multicolumn{1}{c|}{} & Results \\
\hline Terminating Index State & F-Good \\
\hline Experimental Results & $\begin{array}{l}\text { Estimated Function: BurrSize }=0.061+ \\
0.71 C S-0.31 F R+0.46 P A\end{array}$ \\
\hline Data Source Function & $\begin{array}{l}\text { BurrSize }=0.71 C S-0.31 F R+0.46 P A+ \\
\epsilon, \epsilon \sim \mathrm{N}(0,0.01)\end{array}$ \\
\hline Original Number of Samples & 51 \\
\hline Total Sample Cost & 51 \\
\hline Number Of Samples Left & 0 \\
\hline
\end{tabular}

Table 6.49: Final Results of Phase 3 - Test 2

\section{Phase 3 - Test 3}

Function to Approximate: BurrSize $=-0.01 C S * C S+0.05 C S * F R-$ $0.01 F R * F R+\epsilon, \epsilon \sim \mathrm{N}(0,0.01)$

The predictors that passed the screening process are CuttingSpeed and FeedRate. The model is estimated to be BurrSize $=-0.023-0.01 C S * C S+0.05 C S * F R$ $0.01 F R * F R$. The terminating knowledge state index is described in Table 6.50; 


\subsection{Phase 3 - No Task Compromise Minimal Strategy Phase}

\begin{tabular}{|l|l|}
\cline { 2 - 2 } \multicolumn{1}{l|}{} & $\mathrm{i} 0 \mathrm{x} 4 \mathrm{x} 29$ \\
\hline State and Type & F-Good terminating index \\
\hline Samples Available & 3 \\
\hline Obtained Facts & $\begin{array}{l}\text { Potentially Significant Predictors: } \\
C S, F R, P A\end{array}$ \\
& $\begin{array}{l}\text { Sodeened Predictors: } C S, F R \\
C S * C S, C S * F R, F R * F R\end{array}$ \\
\hline Task List & FinishGood \\
\hline Experimental Design & Finish \\
\hline Design Cost & 0 \\
\hline
\end{tabular}

Table 6.50: Descriptions of the knowledge state index during Move 1 of Phase 3 - Test 3

A response function has been successfully modeled, so experimentation can stop, and the final results can be shown. The type of termination, the main experimental results, and the overall cost are displayed in Figure 6.51. 


\subsection{Phase 3 - No Task Compromise Minimal Strategy Phase}

\begin{tabular}{|l|l|}
\cline { 2 - 2 } \multicolumn{1}{c|}{} & Results \\
\hline Terminating Index State & F-Good \\
\hline Experimental Results & $\begin{array}{l}\text { Estimated Function: BurrSize }=-0.023 \text { - } \\
0.01 C S * C S+0.05 C S * F R-0.01 F R * F R\end{array}$ \\
\hline Data Source Function & $\begin{array}{l}\text { BurrSize }=-0.01 C S * C S+0.05 C S * F R \\
-0.01 F R * F R+\epsilon, \epsilon \sim \mathrm{N}(0,0.01)\end{array}$ \\
\hline Original Number of Samples & 51 \\
\hline Total Sample Cost & 48 \\
\hline Number Of Samples Left & 3 \\
\hline
\end{tabular}

Table 6.51: Final Results of Phase 3 - Test 3

\section{Phase 3 - Test 4}

Function to Approximate: BurrSize $=\epsilon, \epsilon \sim \mathrm{N}(0,0.01)$

No predictors passed the screening process. The terminating knowledge state index is described in Table 6.52 


\subsection{Phase 3 - No Task Compromise Minimal Strategy Phase}

\begin{tabular}{|c|c|}
\hline & i0x1 \\
\hline State and Type & F-Good terminating index \\
\hline Samples Available & 35 \\
\hline Obtained Facts & $\begin{array}{l}\text { Potentially Significant Predictors: } \\
C S, F R, P A \\
\text { Screened Vars: None }\end{array}$ \\
\hline Task List & FinishGood \\
\hline Experimental Design & Finish \\
\hline Design Cost & 0 \\
\hline
\end{tabular}

Table 6.52: Descriptions of the knowledge state index during Move 1 of Phase 3 - Test 4

A response function has been successfully modeled, so experimentation can stop, and the final results can be shown. The type of termination, the main experimental results, and the overall cost are displayed in Figure 6.53. 


\subsection{Phase 3 - No Task Compromise Minimal Strategy Phase}

\begin{tabular}{|l|l|}
\cline { 2 - 2 } \multicolumn{1}{c|}{} & Results \\
\hline Terminating Index State & F-Good \\
\hline Experimental Results & Screened predictors of BurrSize: None \\
\hline Data Source Function & BurrSize $=\epsilon, \epsilon \sim \mathrm{N}(0,0.01)$ \\
\hline Original Number of Samples & 51 \\
\hline Total Sample Cost & 16 \\
\hline Number Of Samples Left & 35 \\
\hline
\end{tabular}

Table 6.53: Final Results of Phase 3 - Test 4

\section{Phase 3 Summary:}

This research strategy is F-Good, and is much cheaper than the original research strategy, while still being able to incorporate the predictors of interest. 


\subsection{Phase 4 - Logic Error Phase}

\subsubsection{Preliminary Sponsor-Designer Discussions}

The sponsor likes the results of Example 2, but wants to change the project satisfaction condition to require having a response function constructed containing all variables initially considered, and wants to see what happens. The designer is allowed 90 samples.

\subsubsection{Specifications Expressed Formally by Designer for Phase 4}

Number of samples: 90

\section{Project Satisfaction Condition:}

Response function constructed containing all predictors initially considered

Task Specification Function:

If potential predictors have not gone through screening: $\operatorname{screen}(\operatorname{BurrSize}(C S, F R, P A))$

If potential predictors have gone through screening (in order of preference, greatest to 


\subsection{Phase 4 - Logic Error Phase}

least):

quadraticmodel(BurrSize(screened predictors))

\section{Experimental Design Preference Ordering Function:}

For screening (in order of preference, greatest to least):

Full Factorial Design, 3 replications

Full Factorial Design, 2 replications

For quadratic modeling:

Central Composite Design

maximum number of center points $=($ number of screened predictors +2$) * 2$

minimum number of center points $=$ number of screened predictors +4

maximum number of factorial points $=3$

minimum number of factorial points $=2$

maximum number of axial points $=3$

minimum number of axial points $=2$

start with maximum values, reduce number of center points first, then number of axial points, then number of factorial points 


\subsection{Phase 4 - Logic Error Phase}

\subsubsection{Constructed Research Strategy - Logic Error Phase}

Figure 6.5 is a visual representation of the F-LogErr research strategy, determined from Section 6.5.2:

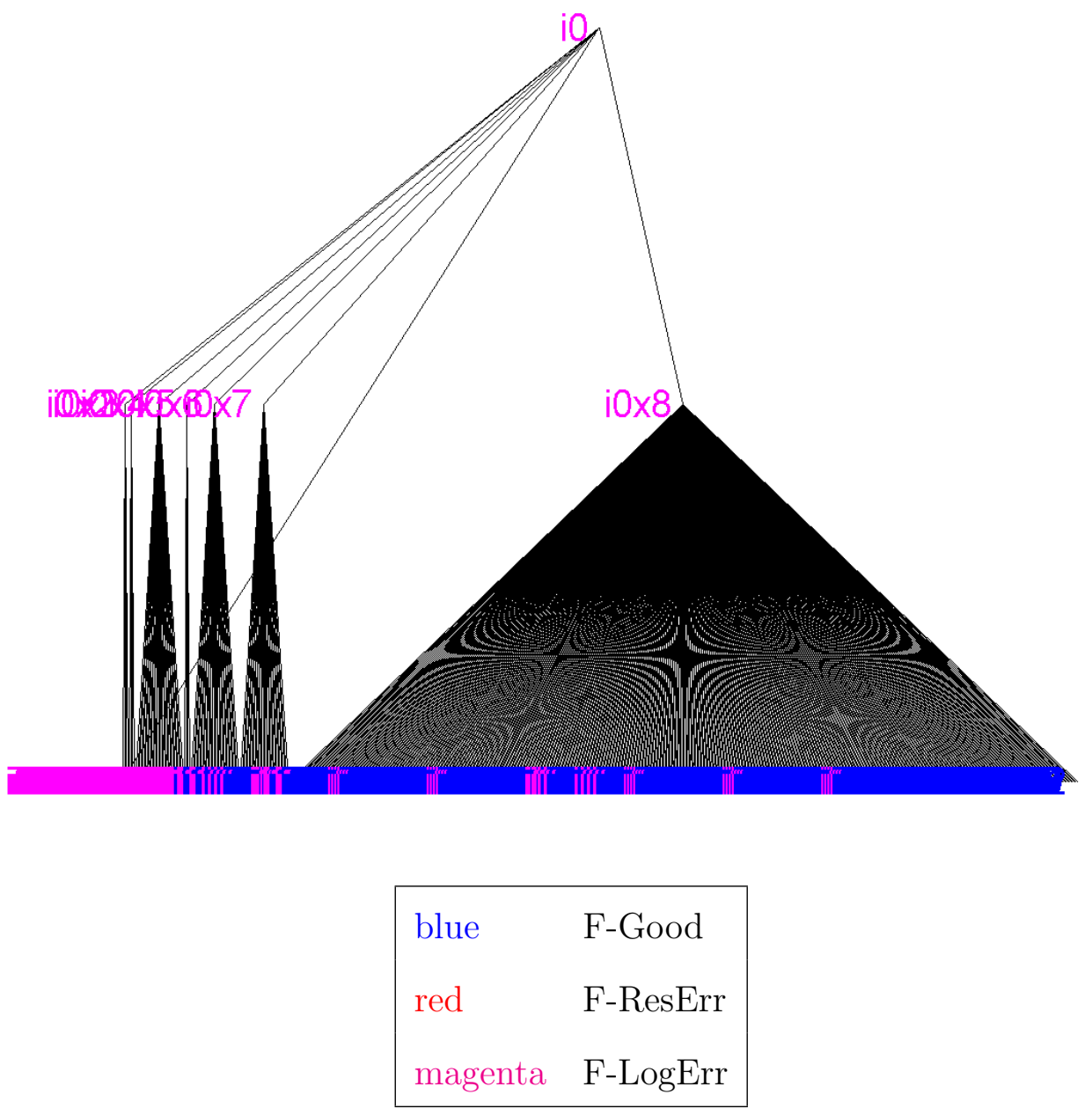

Figure 6.5: Tree graph of research strategy for logic error phase. 


\subsection{Phase 4 - Logic Error Phase}

These are the intermediate knowledge state indexes of the strategy constructed in Phase 4:

\begin{tabular}{|l|l|}
\cline { 2 - 2 } \multicolumn{1}{l|}{} & $\mathrm{i} 0$ \\
\hline State and Type & F-LogErr intermediate index \\
\hline Samples Available & 90 \\
\hline Obtained Facts & $\begin{array}{l}\text { Potentially Significant Predictors: } \\
C S, F R, P A\end{array}$ \\
\hline Task List & screen $(B u r r S i z e(C S, F R, P A))$ \\
\hline Experimental Design & 3 variable 2-level full factorial, 3 reps \\
\hline Design Cost & 24 \\
\hline
\end{tabular}

Table 6.54: Descriptions of the knowledge state index i0 during Phase 4 
6.5 Phase 4 - Logic Error Phase

\begin{tabular}{|c|c|}
\hline & $\mathrm{i} 0 \mathrm{x} 2$ \\
\hline State and Type & F-LogErr intermediate index \\
\hline Samples Available & 66 \\
\hline Obtained Facts & $\begin{array}{l}\text { Potentially Significant Predictors: } \\
C S, F R, P A \\
\text { Screened Predictors: } C S\end{array}$ \\
\hline Task List & quadraticmodel(BurrSize $(C S))$ \\
\hline Experimental Design & $\begin{array}{l}1 \text { variable CCD, } 3 \text { axial reps, } 3 \text { factorial } \\
\text { reps, } 6 \text { center points }\end{array}$ \\
\hline Design Cost & 18 \\
\hline
\end{tabular}

Table 6.55: Descriptions of the knowledge state index i0x2 during Phase 4 
6.5 Phase 4 - Logic Error Phase

\begin{tabular}{|c|c|}
\hline & $\mathrm{i} 0 \times 3$ \\
\hline State and Type & F-LogErr intermediate index \\
\hline Samples Available & 66 \\
\hline Obtained Facts & $\begin{array}{l}\text { Potentially Significant Predictors: } \\
C S, F R, P A \\
\text { Screened Predictors: } F R\end{array}$ \\
\hline Task List & quadraticmodel $($ BurrSize $(F R))$ \\
\hline Experimental Design & $\begin{array}{l}1 \text { variable CCD, } 3 \text { axial reps, } 3 \text { factorial } \\
\text { reps, } 6 \text { center points }\end{array}$ \\
\hline Design Cost & 18 \\
\hline
\end{tabular}

Table 6.56: Descriptions of the knowledge state index i0x3 during Phase 4 
6.5 Phase 4 - Logic Error Phase

\begin{tabular}{|l|l|}
\cline { 2 - 2 } \multicolumn{1}{l|}{} & $\mathrm{i} 0 \mathrm{x} 4$ \\
\hline State and Type & F-LogErr intermediate index \\
\hline Samples Available & 66 \\
\hline Obtained Facts & $\begin{array}{l}\text { Potentially Significant Predictors: } \\
\text { Screened Predictors: } C S, F R\end{array}$ \\
\hline Task List & quadraticmodel $($ Burr $S i z e(C S, F R))$ \\
\hline Experimental Design & 2 variable CCD, 3 axial reps, 3 factorial \\
& reps, 8 center points \\
\hline Design Cost & 32 \\
\hline
\end{tabular}

Table 6.57: Descriptions of the knowledge state index i0x4 during Phase 4 
6.5 Phase 4 - Logic Error Phase

\begin{tabular}{|c|c|}
\hline & $\mathrm{i} 0 \times 5$ \\
\hline State and Type & F-LogErr intermediate index \\
\hline Samples Available & 66 \\
\hline Obtained Facts & $\begin{array}{l}\text { Potentially Significant Predictors: } \\
C S, F R, P A \\
\text { Screened Predictors: } P A\end{array}$ \\
\hline Task List & quadraticmodel $($ BurrSize $(P A))$ \\
\hline Experimental Design & $\begin{array}{l}1 \text { variable CCD, } 3 \text { axial reps, } 3 \text { factorial } \\
\text { reps, } 6 \text { center points }\end{array}$ \\
\hline Design Cost & 18 \\
\hline
\end{tabular}

Table 6.58: Descriptions of the knowledge state index i0x5 during Phase 4 
6.5 Phase 4 - Logic Error Phase

\begin{tabular}{|l|l|}
\cline { 2 - 2 } \multicolumn{1}{l|}{} & i0x6 \\
\hline State and Type & F-LogErr intermediate index \\
\hline Samples Available & 66 \\
\hline Obtained Facts & $\begin{array}{l}\text { Potentially Significant Predictors: } \\
\text { Screened Predictors: } C S, P A\end{array}$ \\
\hline Task List & quadraticmodel $(B u r r S i z e(C S, P A))$ \\
\hline Experimental Design & 2 variable CCD, 3 axial reps, 3 factorial \\
& reps, 8 center points \\
\hline Design Cost & 32 \\
\hline
\end{tabular}

Table 6.59: Descriptions of the knowledge state index i0x6 during Phase 4 
6.5 Phase 4 - Logic Error Phase

\begin{tabular}{|l|l|}
\cline { 2 - 2 } \multicolumn{1}{l|}{} & $\mathrm{i} 0 \mathrm{x} 7$ \\
\hline State and Type & F-LogErr intermediate index \\
\hline Samples Available & 66 \\
\hline Obtained Facts & $\begin{array}{l}\text { Potentially Significant Predictors: } \\
\text { Screened Predictors: } F R, P A\end{array}$ \\
\hline Task List & quadraticmodel $(B u r r S i z e(F R, P A))$ \\
\hline Experimental Design & 2 variable CCD, 3 axial reps, 3 factorial \\
& reps, 8 center points \\
\hline Design Cost & 32 \\
\hline
\end{tabular}

Table 6.60: Descriptions of the knowledge state index i0x7 during Phase 4 
6.5 Phase 4 - Logic Error Phase

\begin{tabular}{|l|l|}
\cline { 2 - 2 } \multicolumn{1}{l|}{} & i0x8 \\
\hline State and Type & F-LogErr intermediate index \\
\hline Samples Available & 66 \\
\hline Obtained Facts & $\begin{array}{l}\text { Potentially Significant Predictors: } \\
\text { Screened Predictors: } C S, F R, P A\end{array}$ \\
\hline Task List & quadraticmodel $(B u r r S i z e(C S, F R, P A))$ \\
\hline Experimental Design & 3 variable CCD, 3 axial reps, 3 factorial \\
& reps, 10 center points \\
\hline Design Cost & 52 \\
\hline
\end{tabular}

Table 6.61: Descriptions of the knowledge state index i0x8 during Phase 4 


\subsection{Phase 4 - Logic Error Phase}

\subsubsection{Model Approximation Testing - Logic Error Phase}

\section{Phase 4 - Test 1}

Function to Approximate: BurrSize $=0.71 C S+0.31 F R+0.46 P A-0.01 C S *$ $C S-0.01 F R * F R-0.01 P A * P A+\epsilon, \epsilon \sim \mathrm{N}(0,0.01)$

The initial knowledge state index is i0, which is described in Table 6.62:

\begin{tabular}{|l|l|}
\cline { 2 - 2 } \multicolumn{1}{c|}{} & i0 \\
\hline State and Type & F-LogErr intermediate index \\
\hline Samples Available & 90 \\
\hline Obtained Facts & $\begin{array}{l}\text { Potentially Significant Predictors: } \\
C S, F R, P A\end{array}$ \\
\hline Task List & screen $($ BurrSize $(C S, F R, P A))$ \\
\hline Experimental Design & 3 variable 2-level full factorial, 3 reps \\
\hline Design Cost & 24 \\
\hline
\end{tabular}

Table 6.62: Descriptions of the knowledge state index during Move 1 of Phase 4 - Test 1

After performing the planned move of i0, the predictors that passed the screening process are CuttingSpeed, FeedRate, and PointAngle. As the result of the experimental events at the previous knowledge state index, the current knowledge state index is now i0x8, which is described in Table 6.63 


\subsection{Phase 4 - Logic Error Phase}

\begin{tabular}{|l|l|}
\cline { 2 - 2 } \multicolumn{1}{l|}{} & i0x8 \\
\hline State and Type & F-LogErr intermediate index \\
\hline Samples Available & 66 \\
\hline Obtained Facts & $\begin{array}{l}\text { Potentially Significant Predictors: } \\
\text { Screened Predictors: } C S, F R, P A\end{array}$ \\
\hline Task List & quadraticmodel $($ Burr $S i z e(C S, F R, P A))$ \\
\hline Experimental Design & 3 variable CCD, 3 axial reps, 3 factorial \\
& reps, 10 center points \\
\hline Design Cost & 52 \\
\hline
\end{tabular}

Table 6.63: Descriptions of the knowledge state index during Move 2 of Phase 4 - Test 1

After performing the planned move of $\mathrm{i} 0 \mathrm{x} 8$, the model is estimated to be BurrSize $=0.079+0.71 C S+0.3 F R+0.46 P A-0.01 C S * C S-9.9 \mathrm{e}-3 F R * F R-0.01 P A * P A$. As the result of the experimental events at the previous knowledge state index, the current knowledge state index is now i0x8x336, which is described in Table 6.64: 


\subsection{Phase 4 - Logic Error Phase}

\begin{tabular}{|l|l|}
\cline { 2 - 2 } \multicolumn{1}{c|}{} & i0x8x336 \\
\hline State and Type & F-Good terminating index \\
\hline Samples Available & 14 \\
\hline Obtained Facts & $\begin{array}{l}\text { Potentially Significant Predictors: } \\
C S, F R, P A \\
\text { Screened Predictors: } C S, F R, P A \\
\text { Modeled Predictors: } \\
C S, F R, P A, C S * C S, F R * F R, P A * P A\end{array}$ \\
\hline Task List & FinishGood \\
\hline Experimental Design & Finish \\
\hline Design Cost & 0 \\
\hline
\end{tabular}

Table 6.64: Descriptions of the knowledge state index during Move 3 of Phase 4 - Test 1

A response function has been successfully modeled, and the model contains all of the predictors of interest, so experimentation can stop, and the final results can be shown. The type of termination, the main experimental results, and the overall cost are displayed in Figure 6.65. 


\subsection{Phase 4 - Logic Error Phase}

\begin{tabular}{|l|l|}
\cline { 2 - 2 } \multicolumn{1}{c|}{} & Results \\
\hline Terminating Index State & F-Good \\
\hline Experimental Results & Estimated Function: BurrSize $=0.079+$ \\
& $0.71 C S+0.3 F R+0.46 P A-0.01 C S * C S$ \\
& $-9.9 \mathrm{e}-3 F R * F R-0.01 P A * P A$ \\
\hline Data Source Function & BurrSize $=0.71 C S+0.31 F R+0.46 P A-$ \\
\hline Original Number of Samples & 90 \\
\hline Total Sample Cost & 76 \\
\hline Number Of Samples Left & 14 \\
\hline
\end{tabular}

Table 6.65: Final Results of Phase 4 - Test 1

\section{Phase 4 - Test 2}

Function to Approximate: BurrSize $=0.71 C S-0.31 F R+0.46 P A+\epsilon, \epsilon$ $\sim \mathrm{N}(0,0.01)$

The predictors that passed the screening process are CuttingSpeed, FeedRate, and PointAngle. The model is estimated to be BurrSize $=0.011+0.71 C S-0.31 F R$ $+0.46 P A+1.4 \mathrm{e}-4 C S * F R$. The terminating knowledge state index is described in Table 6.66: 


\subsection{Phase 4 - Logic Error Phase}

\begin{tabular}{|l|l|}
\cline { 2 - 2 } \multicolumn{1}{l|}{} & $\mathrm{i} 0 \mathrm{x} 8 \mathrm{x} 24$ \\
\hline State and Type & F-Good terminating index \\
\hline Samples Available & 14 \\
\hline Obtained Facts & $\begin{array}{l}\text { Potentially Significant Predictors: } \\
C S, F R, P A\end{array}$ \\
& $\begin{array}{l}\text { Screened Predictors: } C S, F R, P A \\
\text { Modeled Predictors: } C S, F R, P A, C S * F R\end{array}$ \\
\hline Task List & FinishGood \\
\hline Experimental Design & Finish \\
\hline Design Cost & 0 \\
\hline
\end{tabular}

Table 6.66: Descriptions of the knowledge state index during Move 1 of Phase 4 - Test 2

A response function has been successfully modeled, and the model contains all of the predictors of interest, so experimentation can stop, and the final results can be shown. The type of termination, the main experimental results, and the overall cost are displayed in Figure 6.67. 


\subsection{Phase 4 - Logic Error Phase}

\begin{tabular}{|l|l|}
\cline { 2 - 2 } \multicolumn{1}{c|}{} & Results \\
\hline Terminating Index State & F-Good \\
\hline Experimental Results & $\begin{array}{l}\text { Estimated Function: BurrSize }=0.011+ \\
0.71 C S-0.31 F R+0.46 P A+ \\
1.4 \mathrm{e}-4 C S * F R\end{array}$ \\
\hline Data Source Function & BurrSize $=0.71 C S-0.31 F R+0.46 P A+$ \\
\hline Original Number of Samples & 90 \\
\hline Total Sample Cost & 76 \\
\hline Number Of Samples Left & 14 \\
\hline
\end{tabular}

Table 6.67: Final Results of Phase 4 - Test 2

\section{Phase 4 - Test 3}

Function to Approximate: BurrSize $=-0.01 C S * C S+0.05 C S * F R-$ $0.01 F R * F R+\epsilon, \epsilon \sim \mathrm{N}(0,0.01)$

The predictors that passed the screening process are CuttingSpeed and FeedRate. The model is estimated to be BurrSize $=-0.038-0.01 C S * C S+0.05 C S * F R$ $0.01 F R * F R$. The terminating knowledge state index is described in Table 6.68; 


\subsection{Phase 4 - Logic Error Phase}

\begin{tabular}{|l|l|}
\cline { 2 - 2 } \multicolumn{1}{l|}{} & $\mathrm{i} 0 \mathrm{x} 4 \mathrm{x} 29$ \\
\hline State and Type & F-LogErr terminating index \\
\hline Samples Available & 34 \\
\hline Obtained Facts & $\begin{array}{l}\text { Potentially Significant Predictors: } \\
C S, F R, P A \\
\text { Screened Predictors: } C S, F R \\
\text { Modeled Predictors: } \\
C S * C S, C S * F R, F R * F R\end{array}$ \\
\hline Task List & FinishLogErr \\
\hline Experimental Design & Finish \\
\hline Design Cost & 0 \\
\hline
\end{tabular}

Table 6.68: Descriptions of the knowledge state index during Move 1 of Phase 4 - Test 3

A response function for BurrSize has been modeled, but it does not contain all the predictors of interest, which does not meet the project satisfaction condition. Consequently, the project satisfaction function determines that experimentation should continue. However, the available analytical tasks are specified to create a model with predictors that pass the screening and modeling significance tests. Since this been successfully achieved, the specified analytical task list is empty. This means that there are no more tasks to perform, and yet experimentation is supposed to continue. Due to this logical contradiction, experimentation will stop, and the results obtained 


\subsection{Phase 4 - Logic Error Phase}

so far will be given. The type of termination, the main experimental results, and the overall cost are displayed in Figure 6.69.

\begin{tabular}{|l|l|}
\cline { 2 - 2 } \multicolumn{1}{c|}{} & Results \\
\hline Terminating Index State & F-LogErr \\
\hline Experimental Results & $\begin{array}{l}\text { Estimated Function: BurrSize }=-0.038 \text { - } \\
0.01 C S * C S+0.05 C S * F R-0.01 F R * F R\end{array}$ \\
\hline Data Source Function & $\begin{array}{l}\text { BurrSize }=-0.01 C S * C S+0.05 C S * F R \\
-0.01 F R * F R+\epsilon, \epsilon \sim \mathrm{N}(0,0.01)\end{array}$ \\
\hline Original Number of Samples & 90 \\
\hline Total Sample Cost & 56 \\
\hline Number Of Samples Left & 34 \\
\hline
\end{tabular}

Table 6.69: Final Results of Phase 4 - Test 3

\section{Phase 4 - Test 4}

Function to Approximate: BurrSize $=\epsilon, \epsilon \sim \mathrm{N}(0,0.01)$

No predictors passed the screening process. The terminating knowledge state index is described in Table 6.70, 


\subsection{Phase 4 - Logic Error Phase}

\begin{tabular}{|l|l|}
\cline { 2 - 2 } \multicolumn{1}{c|}{} & i0x1 \\
\hline State and Type & F-LogErr terminating index \\
\hline Samples Available & 66 \\
\hline Obtained Facts & $\begin{array}{l}\text { Potentially Significant Predictors: } \\
\text { Screened Vars: None }\end{array}$ \\
\hline Task List & FinishLogErr \\
\hline Experimental Design & Finish \\
\hline Design Cost & 0 \\
\hline
\end{tabular}

Table 6.70: Descriptions of the knowledge state index during Move 1 of Phase 4 - Test 4

All predictors for BurrSize have been screened out, which does not meet the project satisfaction condition. Consequently, the project satisfaction function determines that experimentation should continue. However, the available analytical tasks are specified to create a model with predictors that pass the screening and modeling significance tests. Since this been successfully achieved, the specified analytical task list is empty. This means that there are no more tasks to perform, and yet experimentation is supposed to continue. Due to this logical contradiction, which cannot be resolved experimentally, experimentation will stop, and the results obtained so far will be given. The type of termination, the main experimental results, and the overall cost are displayed in Figure 6.71. 


\subsection{Phase 4 - Logic Error Phase}

\begin{tabular}{|l|l|}
\cline { 2 - 2 } \multicolumn{1}{c|}{} & Results \\
\hline Terminating Index State & F-LogErr \\
\hline Experimental Results & Screened predictors of BurrSize: None \\
\hline Data Source Function & BurrSize $=\epsilon, \epsilon \sim \mathrm{N}(0,0.01)$ \\
\hline Original Number of Samples & 90 \\
\hline Total Sample Cost & 24 \\
\hline Number Of Samples Left & 66 \\
\hline
\end{tabular}

Table 6.71: Final Results of Phase 4 - Test 4

\section{Phase 4 Summary:}

The problem with the new project satisfaction condition is that it only includes knowledge state indexes which represents the case where all variables pass screening and fit the model. The problem with this is that any hypothesis must be falsifiable, and any screening or modeling analytical task can produce a possible fact that will make the new project satisfaction condition unsatisfiable. Therefore, this is not a good project satisfaction condition. 


\subsection{Phase 5 - Catastrophe Phase}

\subsection{Phase 5 - Catastrophe Phase}

\subsubsection{Preliminary Sponsor-Designer Discussions}

The sponsor really didn't like the results of Example 4, but wants to know what happens if samples are accidently lost between performing the first and second experimental designs. The sponsor wants the designer to have 71 samples, and plan for 71 samples, but wants to see what happens if samples disappear between the first and second experimental designs, and wants the designer to compensate for the loss.

\subsubsection{Specifications Expressed Formally by Designer for Phase 5}

Number of samples: 71

\section{Project Satisfaction Condition:}

All potential predictors screened out, or response function constructed

\section{Task Specification Function:}

If potential predictors have not gone through screening:

$\operatorname{screen}(B u r r S i z e(C S, F R, P A))$ 


\subsection{Phase 5 - Catastrophe Phase}

If potential predictors have gone through screening (in order of preference, greatest to least):

quadraticmodel(BurrSize(screened predictors))

\section{Experimental Design Preference Ordering Function:}

For screening (in order of preference, greatest to least):

Full Factorial Design, 3 replications

Full Factorial Design, 2 replications

For quadratic modeling:

Central Composite Design

maximum number of center points $=($ number of screened predictors +2$) * 2$

minimum number of center points $=$ number of screened predictors +4

maximum number of factorial points $=3$

minimum number of factorial points $=2$

maximum number of axial points $=3$

minimum number of axial points $=2$

start with maximum values, reduce number of center points first, then number of axial points, then number of factorial points 


\subsection{Phase 5 - Catastrophe Phase}

\subsubsection{Constructed Research Strategy - Catastrophe Phase}

Figure 6.6 is a visual representation of the F-Good research strategy, determined from Section 6.6.2 (pre-catastrophe):

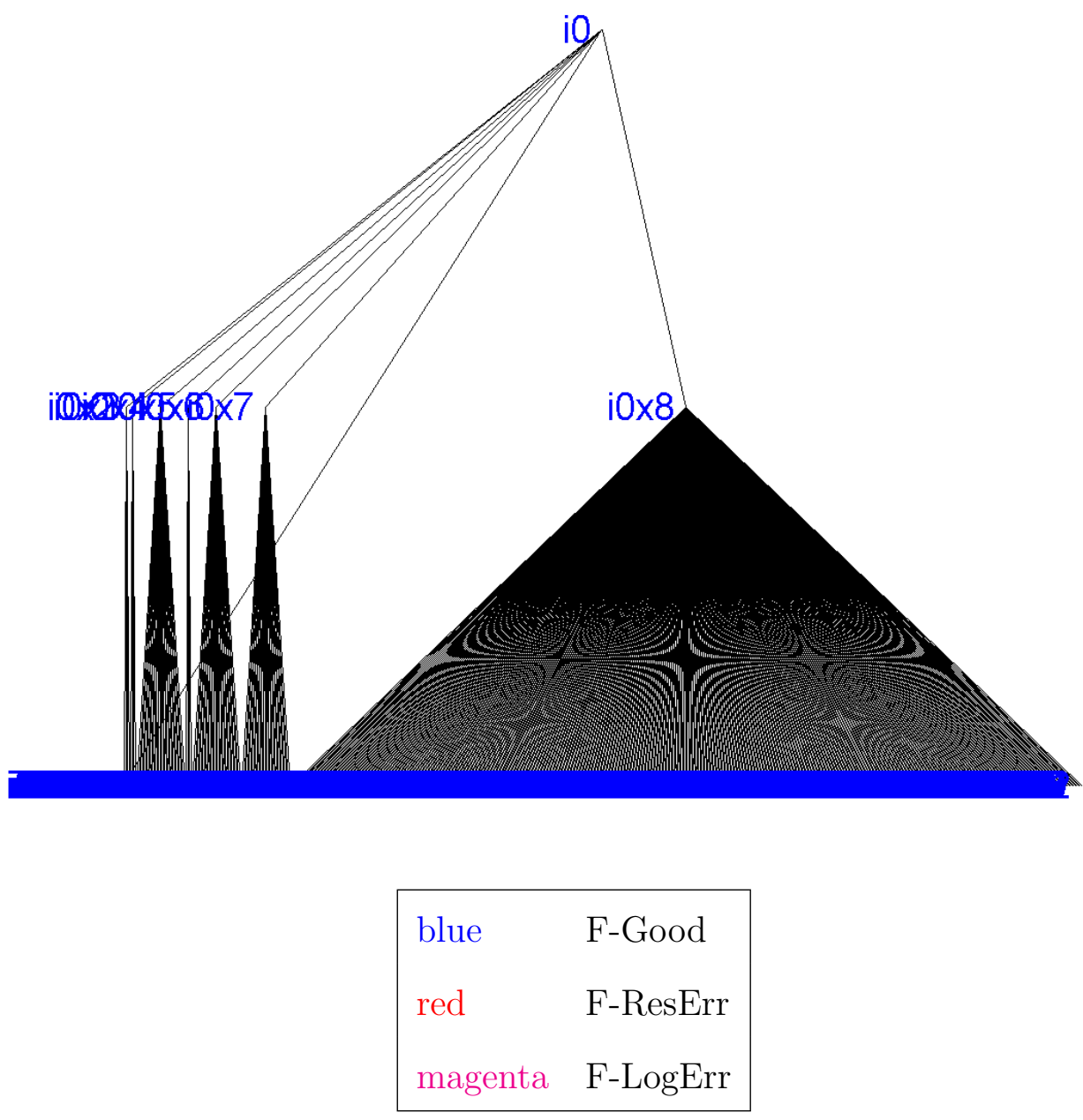

Figure 6.6: Tree graph of research strategy (pre-catastrophe) for catastrophe phase. 


\subsection{Phase 5 - Catastrophe Phase}

These are the intermediate knowledge state indexes of the research strategy constructed in Phase 5(pre-catastrophe):

\begin{tabular}{|l|l|}
\cline { 2 - 2 } \multicolumn{1}{l|}{} & i0 \\
\hline State and Type & F-Good intermediate index \\
\hline Samples Available & 71 \\
\hline Obtained Facts & $\begin{array}{l}\text { Potentially Significant Predictors: } \\
C S, F R, P A\end{array}$ \\
\hline Task List & screen $(B u r r S i z e(C S, F R, P A))$ \\
\hline Experimental Design & 3 variable 2-level full factorial, 3 reps \\
\hline Design Cost & 24 \\
\hline
\end{tabular}

Table 6.72: Descriptions of the knowledge state index i0 during Phase 5(precatastrophe) 
6.6 Phase 5 - Catastrophe Phase

\begin{tabular}{|c|c|}
\hline & $\mathrm{i} 0 \mathrm{x} 2$ \\
\hline State and Type & F-Good intermediate index \\
\hline Samples Available & 47 \\
\hline Obtained Facts & $\begin{array}{l}\text { Potentially Significant Predictors: } \\
C S, F R, P A \\
\text { Screened Predictors: } C S\end{array}$ \\
\hline Task List & quadraticmodel(BurrSize $(C S))$ \\
\hline Experimental Design & $\begin{array}{l}1 \text { variable CCD, } 3 \text { axial reps, } 3 \text { factorial } \\
\text { reps, } 6 \text { center points }\end{array}$ \\
\hline Design Cost & 18 \\
\hline
\end{tabular}

Table 6.73: Descriptions of the knowledge state index i0x2 during Phase 5(pre-catastrophe) 
6.6 Phase 5 - Catastrophe Phase

\begin{tabular}{|c|c|}
\hline & $\mathrm{i} 0 \times 3$ \\
\hline State and Type & F-Good intermediate index \\
\hline Samples Available & 47 \\
\hline Obtained Facts & $\begin{array}{l}\text { Potentially Significant Predictors: } \\
C S, F R, P A \\
\text { Screened Predictors: } F R\end{array}$ \\
\hline Task List & quadraticmodel $($ BurrSize $(F R))$ \\
\hline Experimental Design & $\begin{array}{l}1 \text { variable CCD, } 3 \text { axial reps, } 3 \text { factorial } \\
\text { reps, } 6 \text { center points }\end{array}$ \\
\hline Design Cost & 18 \\
\hline
\end{tabular}

Table 6.74: Descriptions of the knowledge state index i0x3 during Phase 5(pre-catastrophe) 
6.6 Phase 5 - Catastrophe Phase

\begin{tabular}{|l|l|}
\cline { 2 - 2 } \multicolumn{1}{l|}{} & $\mathrm{i} 0 \mathrm{x} 4$ \\
\hline State and Type & F-Good intermediate index \\
\hline Samples Available & 47 \\
\hline Obtained Facts & $\begin{array}{l}\text { Potentially Significant Predictors: } \\
\text { Screened Predictors: } C S, F R\end{array}$ \\
\hline Task List & quadraticmodel(Burr $S i z e(C S, F R))$ \\
\hline Experimental Design & 2 variable CCD, 3 axial reps, 3 factorial \\
& reps, 8 center points \\
\hline Design Cost & 32 \\
\hline
\end{tabular}

Table 6.75: Descriptions of the knowledge state index i0x4 during Phase 5(pre-catastrophe) 
6.6 Phase 5 - Catastrophe Phase

\begin{tabular}{|c|c|}
\hline & $\mathrm{i} 0 \times 5$ \\
\hline State and Type & F-Good intermediate index \\
\hline Samples Available & 47 \\
\hline Obtained Facts & $\begin{array}{l}\text { Potentially Significant Predictors: } \\
C S, F R, P A \\
\text { Screened Predictors: } P A\end{array}$ \\
\hline Task List & quadraticmodel(BurrSize $(P A))$ \\
\hline Experimental Design & $\begin{array}{l}1 \text { variable CCD, } 3 \text { axial reps, } 3 \text { factorial } \\
\text { reps, } 6 \text { center points }\end{array}$ \\
\hline Design Cost & 18 \\
\hline
\end{tabular}

Table 6.76: Descriptions of the knowledge state index i0x5 during Phase 5(pre-catastrophe) 
6.6 Phase 5 - Catastrophe Phase

\begin{tabular}{|l|l|}
\cline { 2 - 2 } \multicolumn{1}{l|}{} & i0x6 \\
\hline State and Type & F-Good intermediate index \\
\hline Samples Available & 47 \\
\hline Obtained Facts & $\begin{array}{l}\text { Potentially Significant Predictors: } \\
\text { Screened Predictors: } C S, P A\end{array}$ \\
\hline Task List & quadraticmodel(Burr Size $(C S, P A))$ \\
\hline Experimental Design & 2 variable CCD, 3 axial reps, 3 factorial \\
& reps, 8 center points \\
\hline Design Cost & 32 \\
\hline
\end{tabular}

Table 6.77: Descriptions of the knowledge state index i0x6 during Phase 5(pre-catastrophe) 
6.6 Phase 5 - Catastrophe Phase

\begin{tabular}{|l|l|}
\cline { 2 - 2 } \multicolumn{1}{l|}{} & $\mathrm{i} 0 \mathrm{x} 7$ \\
\hline State and Type & F-Good intermediate index \\
\hline Samples Available & 47 \\
\hline Obtained Facts & $\begin{array}{l}\text { Potentially Significant Predictors: } \\
\text { Screened Predictors: } F R, P A\end{array}$ \\
\hline Task List & quadraticmodel(Burr Size $(F R, P A))$ \\
\hline Experimental Design & 2 variable CCD, 3 axial reps, 3 factorial \\
& reps, 8 center points \\
\hline Design Cost & 32 \\
\hline
\end{tabular}

Table 6.78: Descriptions of the knowledge state index i0x7 during Phase 5(pre-catastrophe) 
6.6 Phase 5 - Catastrophe Phase

\begin{tabular}{|l|l|}
\cline { 2 - 2 } \multicolumn{1}{l|}{} & $\mathrm{i} 0 \mathrm{x} 8$ \\
\hline State and Type & F-Good intermediate index \\
\hline Samples Available & 47 \\
\hline Obtained Facts & $\begin{array}{l}\text { Potentially Significant Predictors: } \\
\text { Screened Predictors: } C S, F R, P A\end{array}$ \\
\hline Task List & quadraticmodel(Burr $S i z e(C S, F R, P A))$ \\
\hline Experimental Design & 3 variable CCD, 2 axial reps, 3 factorial \\
& reps, 10 center points \\
\hline Design Cost & 46 \\
\hline
\end{tabular}

Table 6.79: Descriptions of the knowledge state index i0x8 during Phase 5(pre-catastrophe) 


\subsection{Phase 5 - Catastrophe Phase}

\subsubsection{Model Approximation Testing - Catastrophe Phase}

\section{Phase 5 - Test 1}

Catastrophic loss of samples at second stage: 5

Function to Approximate: BurrSize $=0.71 C S+0.31 F R+0.46 P A-0.01 C S *$ $C S-0.01 F R * F R-0.01 P A * P A+\epsilon, \epsilon \sim \mathrm{N}(0,0.01)$

The initial knowledge state index is i0, which is described in Table 6.80:

\begin{tabular}{|l|l|}
\cline { 2 - 2 } \multicolumn{1}{c|}{} & i0 \\
\hline State and Type & F-Good intermediate index \\
\hline Samples Available & 71 \\
\hline Obtained Facts & $\begin{array}{l}\text { Potentially Significant Predictors: } \\
C S, F R, P A\end{array}$ \\
\hline Task List & screen $($ BurrSize $(C S, F R, P A))$ \\
\hline Experimental Design & 3 variable 2-level full factorial, 3 reps \\
\hline Design Cost & 24 \\
\hline
\end{tabular}

Table 6.80: Descriptions of the knowledge state index during Move 1 of Phase 5 - Test 1

After performing the planned move of i0, the predictors that passed the screening process are CuttingSpeed, FeedRate, and PointAngle. As the result of the experimental events at the previous knowledge state index, the current knowledge state index is now $\mathrm{i} 0 \mathrm{x} 8$, which is described in Table 6.81. 


\subsection{Phase 5 - Catastrophe Phase}

\begin{tabular}{|l|l|}
\cline { 2 - 2 } \multicolumn{1}{l|}{} & i0x8 \\
\hline State and Type & F-Good intermediate index \\
\hline Samples Available & 47 \\
\hline Obtained Facts & $\begin{array}{l}\text { Potentially Significant Predictors: } \\
\text { Screened Predictors: } C S, F R, P A\end{array}$ \\
\hline Task List & quadraticmodel $($ Burr $S i z e(C S, F R, P A))$ \\
\hline Experimental Design & 3 variable CCD, 2 axial reps, 3 factorial \\
& reps, 10 center points \\
\hline Design Cost & 46 \\
\hline
\end{tabular}

Table 6.81: Descriptions of the knowledge state index during Move 3 of Phase 5 - Test 1

After performing the planned move of $\mathrm{i} 0 \mathrm{x} 8$, the model is estimated to be Burr Size $=-6.6 \mathrm{e}-3+0.71 C S+0.32 F R+0.46 P A-0.01 C S * C S-0.01 F R * F R-0.01 P A * P A$. As the result of the experimental events at the previous knowledge state index, the current knowledge state index is now i0x8x336, which is described in Table 6.82. 


\subsection{Phase 5 - Catastrophe Phase}

\begin{tabular}{|l|l|}
\cline { 2 - 2 } \multicolumn{1}{l|}{} & $\mathrm{i} 0 \mathrm{x} 8 \mathrm{x} 336$ \\
\hline State and Type & F-Good terminating index \\
\hline Samples Available & 1 \\
\hline Obtained Facts & $\begin{array}{l}\text { Potentially Significant Predictors: } \\
C S, F R, P A \\
\text { Screened Predictors: } C S, F R, P A \\
\text { Modeled Predictors: } \\
C S, F R, P A, C S * C S, F R * F R, P A * P A\end{array}$ \\
\hline Task List & FinishGood \\
\hline Experimental Design & Finish \\
\hline Design Cost & 0 \\
\hline
\end{tabular}

Table 6.82: Descriptions of the knowledge state index during Move 5 of Phase 5 - Test 1

A response function has been successfully modeled, so experimentation can stop, and the final results can be shown. The type of termination, the main experimental results, and the overall cost are displayed in Figure 6.83. 


\begin{tabular}{|l|l|}
\cline { 2 - 2 } \multicolumn{1}{c|}{} & Results \\
\hline Terminating Index State & F-Good \\
\hline Experimental Results & Estimated Function: BurrSize $=-6.6 \mathrm{e}-3+$ \\
& $0.71 C S+0.32 F R+0.46 P A-0.01 C S * C S$ \\
& $-0.01 F R * F R-0.01 P A * P A$ \\
\hline Data Source Function & BurrSize $=0.71 C S+0.31 F R+0.46 P A-$ \\
\hline Original Number of Samples & 71 \\
\hline Total Sample Cost & 70 \\
\hline Number Of Samples Left & 1 \\
\hline
\end{tabular}

Table 6.83: Final Results of Phase 5 - Test 1 


\subsection{Phase 5 - Catastrophe Phase}

Phase 5 - Test 2

Catastrophic loss of samples at second stage: 10

Function to Approximate: BurrSize $=0.71 C S+0.31 F R+0.46 P A-0.01 C S *$ $C S-0.01 F R * F R-0.01 P A * P A+\epsilon, \epsilon \sim \mathrm{N}(0,0.01)$

The initial knowledge state index is i0, which is described in Table 6.84:

\begin{tabular}{|l|l|}
\cline { 2 - 2 } \multicolumn{1}{l|}{} & i0 \\
\hline State and Type & F-Good intermediate index \\
\hline Samples Available & 71 \\
\hline Obtained Facts & $\begin{array}{l}\text { Potentially Significant Predictors: } \\
C S, F R, P A\end{array}$ \\
\hline Task List & screen $(B u r r S i z e(C S, F R, P A))$ \\
\hline Experimental Design & 3 variable 2-level full factorial, 3 reps \\
\hline Design Cost & 24 \\
\hline
\end{tabular}

Table 6.84: Descriptions of the knowledge state index during Move 1 of Phase 5 - Test 1

After performing the planned move of i0, the predictors that passed the screening process are CuttingSpeed, FeedRate, and PointAngle. As the result of the experimental events at the previous knowledge state index, the current knowledge state index is now i0x8, which is described in Table 6.85. 


\subsection{Phase 5 - Catastrophe Phase}

\begin{tabular}{|l|l|}
\cline { 2 - 2 } \multicolumn{1}{l|}{} & i0x8 \\
\hline State and Type & F-Good intermediate index \\
\hline Samples Available & 47 \\
\hline Obtained Facts & $\begin{array}{l}\text { Potentially Significant Predictors: } \\
\text { Screened Predictors: } C S, F R, P A\end{array}$ \\
\hline Task List & quadraticmodel $($ Burr $S i z e(C S, F R, P A))$ \\
\hline Experimental Design & 3 variable CCD, 2 axial reps, 3 factorial \\
& reps, 10 center points \\
\hline Design Cost & 46 \\
\hline
\end{tabular}

Table 6.85: Descriptions of the knowledge state index during Move 3 of Phase 5 - Test 1

After performing the planned move of $\mathrm{i} 0 \mathrm{x} 8$, the model is estimated to be Burr Size $=-0.039+0.71 C S+0.31 F R+0.46 P A-0.01 C S * C S-2.2 \mathrm{e}-5 C S * P A-0.01 F R * F R$ - 0.01PA*PA. As the result of the experimental events at the previous knowledge state index, the current knowledge state index is now i0x8x368, which is described in Table 6.86. 


\subsection{Phase 5 - Catastrophe Phase}

\begin{tabular}{|l|l|}
\cline { 2 - 2 } \multicolumn{1}{c|}{} & $\mathrm{i} 0 \mathrm{x} 8 \mathrm{x} 368$ \\
\hline State and Type & F-Good terminating index \\
\hline Samples Available & 1 \\
\hline Obtained Facts & $\begin{array}{l}\text { Potentially Significant Predictors: } \\
C S, F R, P A\end{array}$ \\
& $\begin{array}{l}\text { Screened Predictors: } C S, F R, P A \\
\text { Modeled Predictors: } C S, F R, P A, C S *\end{array}$ \\
\hline Task List & FinishGood $P A, F R * F R, P A * P A$ \\
\hline Experimental Design & Finish \\
\hline Design Cost & 0 \\
\hline
\end{tabular}

Table 6.86: Descriptions of the knowledge state index during Move 5 of Phase 5 - Test 1

A response function has been successfully modeled, so experimentation can stop, and the final results can be shown. The type of termination, the main experimental results, and the overall cost are displayed in Figure 6.87. 


\begin{tabular}{|l|l|}
\cline { 2 - 2 } \multicolumn{1}{l|}{} & Results \\
\hline Terminating Index State & F-Good \\
\hline Experimental Results & Estimated Function: BurrSize $=-0.039+$ \\
& $0.71 C S+0.31 F R+0.46 P A-0.01 C S * C S$ \\
& $-2.2 \mathrm{e}-5 C S * P A-0.01 F R * F R-$ \\
$0.01 P A * P A$
\end{tabular}

Table 6.87: Final Results of Phase 5 - Test 1 


\subsection{Phase 5 - Catastrophe Phase}

Phase 5 - Test 3

Catastrophic loss of samples at second stage: 15

Function to Approximate: BurrSize $=0.71 C S+0.31 F R+0.46 P A-0.01 C S *$ $C S-0.01 F R * F R-0.01 P A * P A+\epsilon, \epsilon \sim \mathrm{N}(0,0.01)$

The initial knowledge state index is i0, which is described in Table 6.88;

\begin{tabular}{|l|l|}
\cline { 2 - 2 } \multicolumn{1}{l|}{} & i0 \\
\hline State and Type & F-Good intermediate index \\
\hline Samples Available & 71 \\
\hline Obtained Facts & $\begin{array}{l}\text { Potentially Significant Predictors: } \\
C S, F R, P A\end{array}$ \\
\hline Task List & screen $(B u r r S i z e(C S, F R, P A))$ \\
\hline Experimental Design & 3 variable 2-level full factorial, 3 reps \\
\hline Design Cost & 24 \\
\hline
\end{tabular}

Table 6.88: Descriptions of the knowledge state index during Move 1 of Phase 5 - Test 1

After performing the planned move of i0, the predictors that passed the screening process are CuttingSpeed, FeedRate, and PointAngle. As the result of the experimental events at the previous knowledge state index, the current knowledge state index is now i0x8, which is described in Table 6.89. 


\subsection{Phase 5 - Catastrophe Phase}

\begin{tabular}{|l|l|}
\cline { 2 - 2 } \multicolumn{1}{l|}{} & i0x8 \\
\hline State and Type & F-Good intermediate index \\
\hline Samples Available & 47 \\
\hline Obtained Facts & $\begin{array}{l}\text { Potentially Significant Predictors: } \\
\text { Screened Predictors: } C S, F R, P A\end{array}$ \\
\hline Task List & quadraticmodel $($ Burr $S i z e(C S, F R, P A))$ \\
\hline Experimental Design & 3 variable CCD, 2 axial reps, 3 factorial \\
& reps, 10 center points \\
\hline Design Cost & 46 \\
\hline
\end{tabular}

Table 6.89: Descriptions of the knowledge state index during Move 3 of Phase 5 - Test 1

After performing the planned move of $\mathrm{i} 0 \mathrm{x} 8$, the model is estimated to be Burr Size $=9.5 \mathrm{e}-3+0.71 C S+0.31 F R+0.46 P A-0.01 C S * C S-0.01 F R * F R-0.01 P A * P A$. As the result of the experimental events at the previous knowledge state index, the current knowledge state index is now i0x8x336, which is described in Table 6.90: 


\subsection{Phase 5 - Catastrophe Phase}

\begin{tabular}{|l|l|}
\cline { 2 - 2 } \multicolumn{1}{l|}{} & $\mathrm{i} 0 \mathrm{x} 8 \mathrm{x} 336$ \\
\hline State and Type & F-Good terminating index \\
\hline Samples Available & 1 \\
\hline Obtained Facts & $\begin{array}{l}\text { Potentially Significant Predictors: } \\
C S, F R, P A \\
\text { Screened Predictors: } C S, F R, P A \\
\text { Modeled Predictors: } \\
C S, F R, P A, C S * C S, F R * F R, P A * P A\end{array}$ \\
\hline Task List & FinishGood \\
\hline Experimental Design & Finish \\
\hline Design Cost & 0 \\
\hline
\end{tabular}

Table 6.90: Descriptions of the knowledge state index during Move 5 of Phase 5 - Test 1

A response function has been successfully modeled, so experimentation can stop, and the final results can be shown. The type of termination, the main experimental results, and the overall cost are displayed in Figure 6.91. 


\subsection{Phase 5 - Catastrophe Phase}

\begin{tabular}{|l|l|}
\cline { 2 - 2 } \multicolumn{1}{c|}{} & Results \\
\hline Terminating Index State & F-Good \\
\hline Experimental Results & Estimated Function: BurrSize $=9.5 \mathrm{e}-3+$ \\
& $0.71 C S+0.31 F R+0.46 P A-0.01 C S * C S$ \\
& $-0.01 F R * F R-0.01 P A * P A$ \\
\hline Data Source Function & BurrSize $=0.71 C S+0.31 F R+0.46 P A-$ \\
\hline Original Number of Samples & 71 \\
\hline Total Sample Cost & 70 \\
\hline Number Of Samples Left & 1 \\
\hline
\end{tabular}

Table 6.91: Final Results of Phase 5 - Test 1

\section{Phase 5 Summary:}

The sponsor is able to observe that even though the minimal strategy cost is 51, as shown in Section 6.4. However, the 71-sample strategy is constructed with the expectation that 71 samples are available, and the planned move for the initial knowledge state index costs more samples as a result. Therefore, a loss of 15 samples at the second stage prevents completion of the project in the case where all variables pass the screening process. The sponsor is willing to accept this, and so accepts the 71-sample research strategy. 


\section{7}

\section{Summary}

\section{$7.1 \quad$ Results}

The key purpose of this research has been to develop a statistical decision-making methodology that can help the researcher to make experimental design choices so as not to waste resources. ARC-RSM is a mathematically sound way to produce deterministically generated, reproducible, testable, defendable, adaptive, resourceconstrained multi-stage experimental schedules without having to spend physical resource, as outlined below:

\section{Deterministically generated:}

Each research strategy is specified by predetermined conditions and preferences, and each step of the process is deterministic (see Chapters 34 ).

\section{Reproducible:}




\subsection{Results}

Every possible planned move of a research strategy uses an existing form of experimental design that is strictly definable in mathematical representations, and is reproducible in the real world (see Section 2.1). In addition, the planned moves of a research strategy are deterministically generated. Therefore, different people using ARC-RSM with the same specifications will end up reproducing the same research strategy.

\section{Testable:}

Since a research strategy uses experimental designs which are applicable in the real world, it is testable in a real-world environment. In addition, since it is mathematically sound and finitely bounded (see Section 3.3 and Sections 4.6 4.8), it can be tested in a simulated environment.

\section{Defendable:}

Each step in generating and using a research strategy is explicitly stated and finitely bounded (see Chapters 34 ), and each choice is restricted to analytical tasks and experimental designs of established literature.

\section{Adaptive:}

The compromise conditions allow planned moves to adjust to feedback during generation (see Section 4.7), and the localized logic at each knowledge index allows adjustment of the research strategy in response to unexpected occurrences (see Section 6.6.4.

\section{Resource-constrained:}

It is a direct requirement of the methodology that the resource constraints be 


\subsection{Current Limitations}

explicitly defined during the specification of the initial knowledge index (see Section 3.3).

\subsection{Current Limitations}

In order to incorporate realistic and pre-existing research limitations, and to ensure terminability, bounding conditions were defined (see Definition 4.22):

- There could only be a finite number of analytical tasks considered at one time, and the list of tasks could only be reduced. If new tasks could be added, then there could potentially be an infinite loop of tasks being added and removed, and terminability could not be assured. Similarly, only a finite number of experimental designs could be considered for each design list, and that list can only go down.

- While an analytical task could return a potentially infinite number of numerical values, that range of values had to be broken into a finite number of partitions, based on what would be considered significant. For example, the range of a significance test would be partitioned into 'pass' and 'fail'. Otherwise, a planned move could have an infinite number of results, making an exhaustive search impossible (in finite time), so terminability could not be assured.

- There has to be a global lower bound for how much an experimental design could cost, or else experimentation could potentially continue forever, since there 


\subsection{Future Research}

could be a sequence experimental design costing half of the resource available to it, so the resource never actually runs out.

In addition, there are also two limiting issues with this methodology:

1. There does not seem to be a general way to incorporate the data of an interrupted experimental design into another experimental design. Therefore, this methodology is limited to starting over with the facts gained by that point and the new amount of resources.

2. This methodology currently uses brute force searches to determine the preferred choice from a reducing algorithm. While it is exhaustive, it can also be potentially expensive computationally.

\subsection{Future Research}

There are three main interests for future research:

1. It would likely be beneficial to use mathematical simplification methods, such as homomorphisms and quotient groups, to convert and reduce complex analytical tasks to more basic structures that would be easier to analyze. For example, $y=a+b^{2}$ might be considered equivalent to $y=a^{2}+b$. Even if there are fine distinctions that are important to the sponsor, a simplified form might be useful in eliminating undesirable planned moves from a reducing algorithm before those distinctions are established. 


\subsection{Future Research}

2. In order to improve the efficiency of task-design selection, it would be of interest to study and compare the performances of different heuristic interpretations and alterations of preference functions. This would allow researchers to develop more efficient but equivalent methods to represent the task-design selection desired by the sponsor-designer team. For example, there can be a rule that no more than a third of the total resources can be spent on screening. This would help designers determine additional criteria for the methodology, beyond what has already been defined, that could improve how quickly a research strategy can be constructed. Furthermore, evolutionary methods like genetic algorithms can be used to further this process, with mathematical methods like topology providing criteria for the evolution of new heuristics.

3. While this methodology has been developed for response surface methodology, it is not inherently limited to it. Therefore, it would be of interest to study how to extend ARC-RSM beyond traditional RSM to other types of statistical analysis, incorporating new tools such as artificial neural networks, fuzzy logic, reconstructability analysis, etc. 


\section{Bibliography}

[1] S. Abbes. The (True) Concurrent Markov Property and Some Applications to Markov Nets, 2005.

[2] D. Ashlock. In Evolutionary Computation for Modeling and Optimization. Springer New York, 2006.

[3] M. Baerns and M. Holeňa. Combinatorial development of solid catalytic materials. London, Imperial College Press, 2009.

[4] S. D. Balkin. A neural network approach to response surface methodology. Communications in Statistics - Theory and Methods, 29:2215-2227, 2000. URL/http://www.personal.psu.edu/users/j/x/jxz203/lin/Lin_pub/ 2000_Commstat2.pdf.

[5] R. Baragona, F. Battaglia, and I. Poli. In Evolutionary Statistical Procedures, Statistics and Computing. Springer Berlin Heidelberg, 2011.

[6] F. Ben Abdelaziz, S. Krichen, and O. Dridi. A Multiobjective Resource- 


\section{BIBLIOGRAPHY}

Constrained Project-Scheduling Problem. In K. Mellouli, editor, Symbolic and Quantitative Approaches to Reasoning with Uncertainty, volume 4724 of Lecture Notes in Computer Science, pages 719-730. Springer Berlin / Heidelberg, 2007. URL http://www.springerlink.com.proxy.lib.pdx.edu/content/ 81533h716k0150q4, xvi, 21, 22

[7] A. Benveniste, E. Fabre, and S. Haar. Markov Nets: Probabilistic Models for distributed and concurrent systems. In IEEE Transactions on Automatic Control, 2001.

[8] M. A. Bezerra, R. E. Santelli, E. P. Oliveira, L. S. Villar, and L. A. Escaleira. Response surface methodology (rsm) as a tool for optimization in analytical chemistry. Talanta, 76(5):965 - 977, 2008. ISSN 0039-9140. doi: DOI:10. 1016/j.talanta.2008.05.019. URL http://www.sciencedirect.com/science/ article/B6THP-4SJP794-8/2/5c82676380bfa8d08c7418c8ab863988.

[9] L. Bianchi, M. Dorigo, L. M. Gambardella, and W. J. Gutjahr. A survey on metaheuristics for stochastic combinatorial optimization. Natural Computing, 8 (2):239-287, 2008.

[10] A. Bobrowski. Functional Analysis for Probability and Stochastic Processes: An Introduction. Cambridge University Press, 2005.

[11] M. Boschetti, V. Maniezzo, and M. Roffilli. Decomposition Techniques as Metaheuristic Frameworks. In V. Maniezzo, T. Sttzle, and S. Vo, editors, 


\section{BIBLIOGRAPHY}

Matheuristics, volume 10 of Annals of Information Systems, pages 135-158. Springer US, 2010. ISBN 978-1-4419-1306-7. URL http://dx.doi.org/10. 1007/978-1-4419-1306-7_5.

[12] G. Box and N. Draper. Response Surfaces, Mixtures, and Ridge Analyses. John Wiley \& Sons, Inc., 2007. xv, 3, 11, 12

[13] G. E. P. Box and K. B. Wilson. On the experimental attainment of optimum conditions. Journal of the Royal Statistical Society. Series B (Methodological), 13(1):pp. 1-45, 1951. ISSN 00359246. URL http://www.jstor.org/stable/ 2983966. 11

[14] J. Branke, S. Greco, R. Slowiński, and P. Zielniewicz. Interactive Evolutionary Multiobjective Optimization Using Robust Ordinal Regression. In Proceedings of the 5th International Conference on Evolutionary Multi-Criterion Optimization, EMO '09, pages 554-568, Berlin, Heidelberg, 2009. Springer-Verlag. ISBN 978-3642-01019-4. doi: http://dx.doi.org/10.1007/978-3-642-01020-0_43. URL http: //dx.doi.org/10.1007/978-3-642-01020-0_43.

[15] D. E. Brown and J. B. Schamburg. A Modified Response Surface Methodology for Knowledge Discovery with Simulations. Journal of Operations Research (Elsevier), 2004. xvi, 17,18

[16] T. Bui and J. Lee. An agent-based framework for building decision support systems. Decis. Support Syst., 25:225-237, April 1999. ISSN 0167- 


\section{BIBLIOGRAPHY}

9236. doi: 10.1016/S0167-9236(99)00008-1. URL http://portal.acm.org/ citation. .fm?id=311624.311629.

[17] S. Cahon, N. Melab, and E. G. Talbi. ParadisEO: A Framework for the Reusable Design of Parallel and Distributed Metaheuristics. Journal of Heuristics, 10(3): 357-380, 2004.

[18] W. Chainbi. Modeling Multi-Agent Systems as Labeled Transitions Systems: A Unifying Approach. In A. Hkansson, N. Nguyen, R. Hartung, R. Howlett, and L. Jain, editors, Agent and Multi-Agent Systems: Technologies and Applications, volume 5559 of Lecture Notes in Computer Science, pages 131140. Springer Berlin / Heidelberg, 2009. URL http://dx.doi.org/10.1007/ 978-3-642-01665-3_14.

[19] R. C. Conant and W. R. Ashby. Every good regulator of a system must be a model of that system. Intl. J. Systems Science, pages 89-97, 1970.

[20] R. C. Conant and W. R. Ashby. Mechanisms of intelligence: Ross Ashby's writings on cybernetics. Intersystems Publications, 1981. xv, 6, 7

[21] M. H. DeGroot. Optimal statistical decisions. New York, McGraw-Hill, 1970.

[22] G. Deng. Simulation-based optimization, 2007. URL http://pages.cs.wisc. edu/ ferris/theses/GengDeng.pdf.

[23] D. Dhupal, B. Doloi, and B. Bhattacharyya. Optimization of process parameters of nd:yag laser microgrooving of al2tio5 ceramic material by 


\section{BIBLIOGRAPHY}

response surface methodology and artificial neural network algorithm. Proceedings of the Institution of Mechanical Engineers - Part B - Engineering Manufacture, 221(8):1341 - 1350, 2007. ISSN 09544054. URL http://stats.lib.pdx.edu.proxy.lib.pdx.edu/proxy.php?url=http:

//search.ebscohost.com.proxy.lib.pdx.edu/login.aspx?direct=true\& $\mathrm{db}=\mathrm{aph} \& \mathrm{AN}=26369518 \&$ site=ehost-live.

[24] J. Dowling and S. Haridi. Decentralized Reinforcement Learning for the Online Optimization of Distributed Systems. In M. E. Cornelius Weber and N. M. Mayer, editors, Reinforcement Learning: Theory and Applications, Advanced Robotic Systems Journal, pages 143-166. I-Tech Education and Publishing, Vienna, Austria. ISBN 978-3-902613-14-1. URL http://www. intechopen.com/ articles/show/title/decentralized_reinforcement_learning_for_the_ online_optimization_of_distributed_systems.

[25] B. Dubiel and O. Tsimhoni. Integrating agent based modeling into a discrete event simulation. In Proceedings of the 37th conference on Winter simulation, WSC '05, pages 1029-1037. Winter Simulation Conference, 2005. ISBN 0-78039519-0. URL http://portal .acm.org/citation . cfm?id=1162708.1162888.

[26] M. G. Eboli and F. G. Cozman. Markov decision processes from colored Petri nets. In Proceedings of the 20th Brazilian conference on Advances in artificial intelligence, SBIA'10, pages 72-81, Berlin, Heidelberg, 2010. Springer- 


\section{BIBLIOGRAPHY}

Verlag. ISBN 3-642-16137-5, 978-3-642-16137-7. URL http://portal .acm.org/ citation. cfm?id=1929622.1929631.

[27] A. Ebrahimpour, R. Rahman, D. Ean Ch'ng, M. Basri, and A. Salleh. A modeling study by response surface methodology and artificial neural network on culture parameters optimization for thermostable lipase production from a newly isolated thermophilic geobacillus sp. strain arm. BMC Biotechnology, 8(1):96, 2008. ISSN 1472-6750. doi: 10.1186/1472-6750-8-96. URL http://www.biomedcentral. com/1472-6750/8/96.

[28] C. Ermel and A. Martini. A Taste of Categorical Petri Nets, 1996. URL http: //citeseerx.ist.psu.edu/viewdoc/summary?doi=10.1.1.35.2086.

[29] J. Ferreirós. Consolidation of axiomatic set theory. In Labyrinth of Thought, pages 365-392. Birkhäuser Basel, 2007. ISBN 978-3-7643-8350-3. URL http: //dx.doi.org/10.1007/978-3-7643-8350-3_11.

[30] K. Fleszar and K. S. Hindi. Solving the resource-constrained project scheduling problem by a variable neighbourhood search. European Journal of Operational Research, 155(2):402 - 413, 2004. ISSN 0377-2217. doi: DOI:10.1016/ S0377-2217(02)00884-6. URL http://www.sciencedirect.com/science/ article/B6VCT-48326GK-8/2/b526d371f6e9916794470aab060331ea. 22

[31] D. M. Gabbay and K. Schlechta. Logical Tools for Handling Change in AgentBased Systems. Cognitive Technologies. Springer Berlin Heidelberg, 2010. ISBN 


\section{BIBLIOGRAPHY}

978-3-642-04407-6. URL http://dx.doi.org/10.1007/978-3-642-04407-6_ 1 .

[32] P. Gács, J. T. Tromp, and P. M. Vitányi. Algorithmic Statistics. IEEE Transactions on Information Theory, 47:2443-2463, 2001.

[33] M. H. Gadallah. Optimization Formulation Based on Limited Data and RSM: An Approximation.

[34] A. Georgieva and I. Jordanov. Hybrid Metaheuristics for Global Optimization: A Comparative Study. In Proceedings of the 3rd international workshop on Hybrid Artificial Intelligence Systems, HAIS '08, pages 298-305, Berlin, Heidelberg, 2008. Springer-Verlag. ISBN 978-3-540-87655-7. doi: http:// dx.doi.org/10.1007/978-3-540-87656-4_37. URL http://dx.doi.org/10.1007/ 978-3-540-87656-4_37.

[35] C. Gershenson. Design and Control of Self-Organizing Systems, 2007. URL http://citeseerx.ist.psu.edu/viewdoc/download?doi=10.1.1.88.2924\& rep=rep1\&type $=p d f$.

[36] N. Gilbert and K. G. Troitzsch. Simulation for the Social Scientist. Maidenhead, New York, Open University Press, 2005.

[37] P. C. Giordano, H. D. Marténez, A. A. Iglesias, A. J. Beccaria, and H. C. Goicoechea. Application of response surface methodology and artificial neural networks for optimization of recombinant oryza sativa non-symbiotic hemoglobin 


\section{BIBLIOGRAPHY}

1 production by escherichia coli in medium containing byproduct glycerol. Bioresource Technology, 101(19):7537 - 7544, 2010. ISSN 0960-8524. doi: DOI:10. 1016/j.biortech.2010.05.001. URL http://www.sciencedirect.com/science/ article/B6V24-504W3SK-4/2/007c45d816dd9069f7c6bb776d50c906.

[38] A. K. Gupta. Predictive modelling of turning operations using response surface methodology, artificial neural networks and support vector regression. International Journal of Production Research, 48(3):763 - 778, 2010. ISSN 00207543. URL http://stats.lib.pdx.edu.proxy.lib.pdx.edu/proxy. php?url=http://search.ebscohost.com.proxy.lib.pdx.edu/login.aspx? direct=true\&db=buh\&AN=49143836\&site=ehost - live.

[39] W. J. Gutjahr. Convergence analysis of metaheuristics. In V. Maniezzo, T. Sttzle, and S. Vo, editors, Matheuristics, volume 10 of Annals of Information Systems, pages 159-187. Springer US, 2010. ISBN 978-1-4419-1306-7. URL http://www. springerlink.com.proxy.lib.pdx.edu/content/r62237182k2k2557.

[40] G. Hanrahan and K. Lu. Application of factorial and response surface methodology in modern experimental design and optimization. Critical Reviews in Analytical Chemistry, 36(3/4):141 - 151, 2006. ISSN 10408347. URL http://stats.lib.pdx.edu.proxy.lib.pdx.edu/proxy.php?url=http: //search.ebscohost.com.proxy.lib.pdx.edu/login.aspx?direct=true\& $\mathrm{db}=$ aph\&AN=23332261\&site=ehost-live.

[41] S. Hess and S. von Cramon-Taubadel. Meta Response Surface Design for General 


\section{BIBLIOGRAPHY}

and Partial Equilibrium Models. 2008 International Congress, August 26-29, 2008, Ghent, Belgium 43689, European Association of Agricultural Economists, 2008. URL http://ideas .repec.org/p/ags/eaae08/43689.html.

[42] D. G. Humphrey and J. R. Wilson. A Revised Simplex Search Procedure for Stochastic Simulation Response Surface Optimization. INFORMS JOURNAL ON COMPUTING, 12(4):272-283, 2000. doi: 10.1287/ijoc.12.4.272.11879. URL http://joc. journal. informs.org/cgi/content/abstract/12/4/272.

[43] A. Jaszkiewicz. Evaluation of Multiple Objective Metaheuristics. In M. S. K. T. V. Gandibleux, X.; Sevaux, editor, Metaheuristics for Multiobjective Optimisation, volume 535 of Lecture Notes in Economics and Mathematical Systems, pages 65-89. Berlin ; New York, N.Y. : Springer, c2004., 2004. ISBN 978-3-54020637-8.

[44] A. Jaszkiewicz and J. Branke. Interactive Multiobjective Evolutionary Algorithms. In J. Branke, K. Deb, K. Miettinen, and R. Slowinski, editors, Multiobjective Optimization, volume 5252 of Lecture Notes in Computer Science, pages 179-193. Springer Berlin / Heidelberg, 2008. URL http://dx.doi.org/ 10.1007/978-3-540-88908-3_7.

[45] S. Karnik, V. Gaitonde, and J. Davim. A comparative study of the ann and rsm modeling approaches for predicting burr size in drilling. The International Journal of Advanced Manufacturing Technology, 38:868-883, 2008. ISSN 02683768. URL http://dx.doi.org/10.1007/s00170-007-1140-7. 70 


\section{BIBLIOGRAPHY}

[46] M. B. Kasiri, H. Aleboyeh, and A. Aleboyeh. Modeling and optimization of heterogeneous photo-fenton process with response surface methodology and artificial neural networks. Environmental Science \& Technology, 42(21):7970-7975, 2008. doi: 10.1021/es801372q. URL http://pubs.acs.org/doi/abs/10.1021/ es801372q.

[47] J. R. Kathleen M. Carley, Natalia Y. Kamneva. Response Surface MethodologyCASOS Technical Report, 2004. URL http://reports-archive.adm.cs.cmu. edu/anon/isri2004/CMU-ISRI-04-136.pdf.

[48] D. Kim, S. Rhee, and H. Park. Modelling and optimization of a gma welding process by genetic algorithm and response surface methodology. International Journal of Production Research, 40(7):1699 - 1711, 2002. ISSN 00207543. URL http://stats.lib.pdx.edu.proxy.lib.pdx.edu/proxy.php?url=http: //search.ebscohost.com.proxy.lib.pdx.edu/login.aspx?direct=true\& $\mathrm{db}=$ buh\&AN=6638925\&site=ehost - live.

[49] J. P. Kleijnen. Response Surface Methodology for Constrained Simulation Optimization: an Overview. Simulation Modelling Practice and Theory, 16(1):50-64, 2008.

[50] J. R. Kok and N. Vlassis. Collaborative Multiagent Reinforcement Learning by Payoff Propagation. JOURNAL OF MACHINE LEARNING RESEARCH, 7: $1789-1828,2006$. 


\section{BIBLIOGRAPHY}

[51] R. Kolisch and S. Hartmann. Heuristic Algorithms for Solving the ResourceConstrained Project Scheduling Problem: Classification and Computational Analysis. Project scheduling: Recent models, algorithms and applications, pages 147-178, 1999. 22

[52] R. Kolisch and S. Hartmann. Experimental evaluation of state-ofthe-art heuristics for the resource-constrained project scheduling problem. European Journal of Operational Research, 127(2):394 - 407, 2000. ISSN 0377-2217. doi: DOI:10.1016/S0377-2217(99)00485-3. URL http://www.sciencedirect.com/science/article/B6VCT-419JGY3-G/ 2/9c53398b4f51f869c28decea7b0c6b23. 22

[53] R. Kolisch and S. Hartmann. Experimental Investigation of Heuristics for Resource-Constrained Project Scheduling: An Update. European Journal of Operational Research, 174(1):23 - 37, 2006. ISSN 0377-2217. doi: DOI: 10.1016/j.ejor.2005.01.065. URL http://www.sciencedirect.com/science/ article/B6VCT-4G7DY5D-7/2/6b51533fd69866a5f3a3a556e13ea72a, 22

[54] J. Lancaster and M. Ozbayrak. Evolutionary algorithms applied to project scheduling problems - a survey of the state-of-the-art. International Journal of Production Research, 45(2):425 - 450, 2007. ISSN 00207543. URL http://stats.lib.pdx.edu.proxy.lib.pdx.edu/proxy.php?url=http: //search.ebscohost.com.proxy.lib.pdx.edu/login.aspx?direct=true\& $\mathrm{db}=$ buh\&AN $=24155431$ \&s ite=ehost - live. 


\section{BIBLIOGRAPHY}

[55] H. C. Lau, W. C. Wan, M. K. Lim, and S. Halim. A Development Framework for Rapid Metaheuristics Hybridization. In Proc. Metaheuristics: Progress as Real Problem Solvers 28th Annual International Computer Software and Applications Conference (COMPSAC), 362-367, Hong Kong, pages 362-367, 2004.

[56] A. M. Law and M. G. McComas. Simulation-based optimization. In Proceedings of the 32nd conference on Winter simulation, WSC '00, pages 46-49, San Diego, CA, USA, 2000. Society for Computer Simulation International. ISBN 0-78036582-8. URL http://portal.acm.org/citation. cfm?id=510378.510389.

[57] J. Liu, W. Zhong, and L. Jiao. Multi-Agent Evolutionary Model for Global Numerical Optimization. In L. M. Hiot, Y. S. Ong, R. A. Sarker, and T. Ray, editors, Agent-Based Evolutionary Search, volume 5 of Adaptation, Learning, and Optimization, pages 13-48. Springer Berlin Heidelberg, 2010. ISBN 978-3-64213425-8. URL http://dx.doi.org/10.1007/978-3-642-13425-8_2.

[58] R. Martí and G. Reinelt. Meta-Heuristics. In S. S. Antman, P. Holmes, L. Sirovich, and K. Sreenivasan, editors, The Linear Ordering Problem, volume 175 of Applied Mathematical Sciences, pages 41-84. Springer Berlin Heidelberg, 2011. ISBN 978-3-642-16729-4. URL http://dx.doi.org/10.1007/ 978-3-642-16729-4_3.

[59] A. Martini. Elements of Basic Category Theory. Technical report, 1996.

[60] G. Merkuryeva. Response Surface-Based Simulation Metamodelling Methods. 


\section{BIBLIOGRAPHY}

In P. M. Pardalos, D. Hearn, A. Dolgui, J. Soldek, and O. Zaikin, editors, Supply Chain Optimisation, volume 94 of Applied Optimization, pages 205-215. Springer US, 2005. ISBN 978-0-387-23581-3. URL http://dx.doi.org/10. 1007/0-387-23581-7_15.

[61] B. Meyer. Hybrids of Constructive Metaheuristics and Constraint Programming: A Case Study with ACO. In C. Blum, M. Aguilera, A. Roli, and M. Sampels, editors, Hybrid Metaheuristics, volume 114 of Studies in Computational Intelligence, pages 151-183. Springer Berlin / Heidelberg, 2008. ISBN 978-3-540-782940. URL http://dx.doi.org/10.1007/978-3-540-78295-7_6.

[62] D. C. Montgomery. Design and analysis of experiments. John Wiley \& Sons, 2008. xv, 8, 13

[63] R. H. Myers, A. I. Khuri, and J. Carter, Walter H. Response surface methodology: 1966-1988. Technometrics, 31(2):pp. 137-157, 1989. ISSN 00401706. URL http://www.jstor.org/stable/1268813.

[64] R. H. Myers, D. C. Montgomery, and C. M. Anderson-Cook. Response Surface Methodology. John Wiley \& Sons, Inc., 2009.

[65] V. Nannen and A. E. Eiben. Efficient relevance estimation and value calibration of evolutionary algorithm parameters. In IEEE Congress on Evolutionary Computation '07, pages 103-110, 2007.

[66] H. G. Neddermeijer, G. J. van Oortmarssen, N. Piersma, and R. Dekker. A 


\section{BIBLIOGRAPHY}

framework for Response Surface Methodology for simulation optimization. In Proceedings of the 32nd conference on Winter simulation, WSC '00, pages 129136, San Diego, CA, USA, 2000. Society for Computer Simulation International. ISBN 0-7803-6582-8. URL http://portal.acm.org/citation.cfm?id= 510378.510401. xvi, 15, 16

[67] M. K. Neelam Shihani, B. K. Kumbhar. Modeling of extrusion process using response surface methodology and artificial neural networks. Journal of Engineering Science and Technology, 1(1):31-40, 2006.

[68] R. Nicolai and R. Dekker. Automated Response Surface Methodology for Simulation Optimization Models with Unknown Variance. Quality Technology \& Quantitative Management, 6:325-352, 2009. xvi, 19, 20

[69] R. Noorossana, S. Davanloo Tajbakhsh, and A. Saghaei. An artificial neural network approach to multiple-response optimization. The International Journal of Advanced Manufacturing Technology, 40:1227-1238, 2009. ISSN 0268-3768. URL http://dx.doi .org/10.1007/s00170-008-1423-7.

[70] M. J. North and C. M. Macal. Managing Business Complexity: Discovering Strategic Solutions with Agent-Based Modeling and Simulation. Oxford University Press, Inc., 2007.

[71] R. Olfati-saber, J. A. Fax, and R. M. Murray. Consensus and cooperation in 


\section{BIBLIOGRAPHY}

networked multi-agent systems. In Proceedings of the IEEE, volume 95, pages 215-233, 2007.

[72] T. Ören. Software Agents for Experimental Design in Advanced Simulation Environments. Proc. of the 4th St. Petersburg Workshop on Simulation, June 18-23, 2001, pages 89-95, 2001.

[73] M. J. Osborne and A. Rubinstein. A course in game theory. MIT Press, 1994. 22

[74] D. Ouelhadj and S. Petrovic. A cooperative distributed hyper-heuristic framework for scheduling. 2008 IEEE International Conference on Systems, Man and Cybernetics (SMC 2008), pages 2560 - 2565, 2008. 22

[75] S. Parsons and M. Wooldridge. Game Theory and Decision Theory in MultiAgent Systems. Autonomous Agents and Multi-Agent Systems, 5:243-254, September 2002. ISSN 1387-2532. doi: 10.1023/A:1015575522401. URL http://portal.acm.org/citation. cfm?id=608611.608694. 23

[76] I. Partalas, I. Feneris, and I. P. Vlahavas. A Hybrid Multiagent Reinforcement Learning Approach Using Strategies and Fusion. International Journal on Artificial Intelligence Tools.

[77] S. Prestwich. The Relation Between Complete and Incomplete Search. In C. Blum, M. Aguilera, A. Roli, and M. Sampels, editors, Hybrid Metaheuristics, volume 114 of Studies in Computational Intelligence, pages 63- 


\section{BIBLIOGRAPHY}

83. Springer Berlin / Heidelberg, 2008. URL http://dx.doi.org/10.1007/ 978-3-540-78295-7_3.

[78] B. Rahmanian, M. Pakizeh, S. A. A. Mansoori, and R. Abedini. Application of experimental design approach and artificial neural network (ann) for the determination of potential micellar-enhanced ultrafiltration process. Journal of Hazardous Materials, 187(1-3):67 - 74, 2011. ISSN 0304-3894. doi: DOI:10. 1016/j.jhazmat.2010.11.135. URL http://www.sciencedirect.com/science/ article/B6TGF-51N7RWV-9/2/38538024a838936c20401e126506a545.

[79] M. Rørdam, F. Larsen, and N. Laustsen. An introduction to K-theory for $C^{*}$ algebras. Cambridge University Press, 2000.

[80] J. B. Schamburg and D. E. Brown. A generalized multiple response surface methodology for complex computer simulation applications. In Proceedings of the 36th conference on Winter simulation, WSC '04, pages 958-966. Winter Simulation Conference, 2004. ISBN 0-7803-8786-4. URL http://portal.acm. org/citation.cfm?id=1161734.1161909. 17

[81] K. Taveter and G. Wagner. Agent-Oriented Enterprise Modeling Based on Business Rules. In IN PROC. OF 2OTH INT. CONF. ON CONCEPTUAL MODELING (ER2001, pages 527-540. Springer-Verlag, 2001.

[82] S. A. Umpleby. Ross ashby's general theory of adaptive systems. International Journal of General Systems, 38(2):231 - 238, 2009. ISSN 03081079. URL 


\section{BIBLIOGRAPHY}

http://stats.lib.pdx.edu.proxy.lib.pdx.edu/proxy.php?url=http:

//search.ebscohost.com.proxy.lib.pdx.edu/login.aspx?direct=true\&

$\mathrm{db}=\mathrm{a} 9 \mathrm{~h} \& \mathrm{AN}=36323761 \&$ site $=$ ehost - live.

[83] V. Valls, S. Quintanilla, and F. Ballestin. An Evolutionary Approach to the Resource-Constrained Project Scheduling Problem. Annals of Operations Research, 131:2004, 2001.

[84] A. van Breemen and T. D. Vries. An Agent-Based Framework for Designing Multi-Controller Systems. In Proc. of the Fifth International Conference on The Practical Applications of Intelligent Agents and Multi-Agent Technology, pages 219-235, 2000.

[85] G. Verfaillie, C. Pralet, and M. Lemaître. Constraint-based modeling of discrete event dynamic systems. Journal of Intelligent Manufacturing, 21:31-47, 2010. ISSN 0956-5515. URL http://dx.doi.org/10.1007/s10845-008-0176-3.

[86] L. Wang, S. J. Turner, and F. Wang. Resolving mutually exclusive interactions in agent based distributed simulations. In Proceedings of the 36th conference on Winter simulation, WSC '04, pages 783-791. Winter Simulation Conference, 2004. ISBN 0-7803-8786-4. URL http://portal.acm.org/citation.cfm?id= 1161734.1161877.

[87] L. F. Wilson, D. Burroughs, J. Sucharitaves, and A. Kumar. An agent-based framework for linking distributed simulations. In Proceedings of the 32nd con- 


\section{BIBLIOGRAPHY}

ference on Winter simulation, WSC '00, pages 1713-1721, San Diego, CA, USA, 2000. Society for Computer Simulation International. ISBN 0-7803-6582-8. URL http://portal.acm.org/citation. cfm?id=510378.510630.

[88] D. Wolf, O. V. Buyevskaya, and M. Baerns. An evolutionary approach in the combinatorial selection and optimization of catalytic materials. Applied Catalysis A: General, 200(1-2):63 - 77, 2000. ISSN 0926-860X. doi: DOI:10.1016/ S0926-860X(00)00643-8. URL http://www.sciencedirect.com/science/ article/B6TF5-4105N1D-6/2/7855a38fc180d6856de8052898d8aa2f.

[89] R. Yang, P.-A. Heng, and K.-S. Leung. Backward Reasoning on Rule-Based Systems Modeled by Fuzzy Petri Nets Through Backward Tree. In S. K. Halgamuge and L. Wang, editors, Computational Intelligence for Modelling and Prediction, volume 2 of Studies in Computational Intelligence, pages 61-71. Springer Berlin / Heidelberg, 2005. URL http://dx.doi.org/10.1007/10966518_5.

[90] D. Ye, M. Zhang, and D. Sutanto. DGF: Decentralized Group Formation for Task Allocation in Complex Adaptive Systems. In Q. Bai and N. Fukuta, editors, Advances in Practical Multi-Agent Systems, volume 325 of Studies in Computational Intelligence, pages 3-19. Springer Berlin / Heidelberg, 2011. URL http://dx.doi.org/10.1007/978-3-642-16098-1_1.

[91] P. V. Yee and S. Haykin. Regularized Radial Basis Function Networks. John Wiley \& Sons, Inc., 2001. 


\section{BIBLIOGRAPHY}

[92] L. Yilmaz and T. Ören. Agent-Directed Simulation and Systems Engineering. WILEY-VCH Verlag GmbH \& Co. KGaA, Weinheim, 2009.

[93] S. Youssefi, Z. Emam-Djomeh, and S. M. Mousavi. Comparison of artificial neural network (ann) and response surface methodology (rsm) in the prediction of quality parameters of spray-dried pomegranate juice. Drying Technology, 27(7/8):910 - 917, 2009. ISSN 07373937. URL http://stats.lib.pdx.edu.proxy.lib.pdx.edu/proxy.php?url=http:

//search.ebscohost.com.proxy.lib.pdx.edu/login.aspx?direct=true\& $\mathrm{db}=$ aph\&AN=43211469\&site=ehost - live.

[94] S. Zacks. Stage-Wise Adaptive Designs. Hoboken, N.J., John Wiley \& Sons, Inc., 2009 .

[95] Z. Zhang and C. Zhang. Agent-Based Hybrid Intelligent Systems, volume 2938 of Lecture Notes in Computer Science. Springer Berlin / Heidelberg, 2004. URL http://www.springerlink.com.proxy.lib.pdx.edu/content/ 978-3-540-20908-9/. 


\section{Appendix A}

\section{Additional Background}

\section{A.1 Design of Experiments}

Definition A.1. A response is a main element of interest within a system that a researcher is trying to control, but cannot manipulate directly. When trying to affect a given response, an element of interest which can be directly controlled and is suspected of having a direct effect on the response is called a predictor. An experimental run is an observance of the response variable as the values of the predictor variables are changed or replicated. An element which may affect the response, but is not of interest to the experimenter, is called a nuisance factor. An experimental design is a schedule of experimental runs arranged in order to isolate specific effects and reduce the effects of nuisance factors.

Definition A.2. Blocking is a type of experimental design technique in which ex- 


\section{A.1 Design of Experiments}

perimental runs are arranged in order to reduce specific nuisance factors.

Definition A.3. Screening is a process which can be performed using experimental design, which is intended to determine which predictors have a significant effect on the response.

Definition A.4. Modeling is a process which can be performed using experimental design, which is intended to determine the relationship the predictors have on the response, including the effects that the interrelationships of the predictors have on the response. This relationship includes a response function, which is a function which outputs a prediction of the response based upon the predictor values.

Definition A.5. Optimization is a process which can be performed using experimental design, and which requires an existing response function, uses the current response function with new experimental data to derive an estimate for the predictor values most likely to result in an optimal value for the response.

Definition A.6. A central composite design is a kind of experimental design typically used for fitting a second-order model. An example of a 2-factor central composite design is shown in Figure A.1. 


\section{A.1 Design of Experiments}

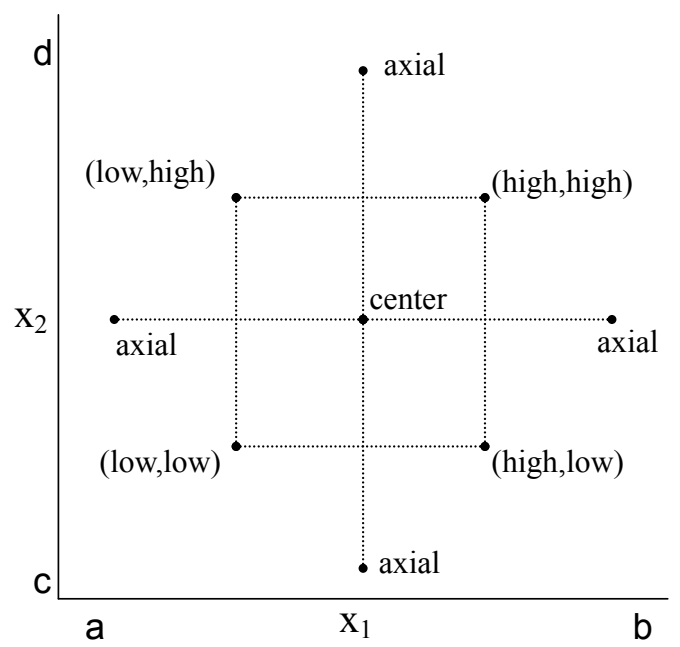

Figure A.1: Example of a central composite design with two predictors.

The point at the center of the design is referred to as a center point, the points of the square are referred to as corner points (the name can vary), and the points at the end of the cross are axial points.

Definition A.7. A factorial design is a kind of experimental design with a variety of uses (screening is one of the most common), and can be embedded in more complicated experimental designs. A factorial design has $k$ predictors, and each predictor has $n$ levels. In a full factorial design, also called a $\boldsymbol{n}^{\boldsymbol{k}}$ factorial design, each possible combination of predictor levels is tested the same number of times as the others. An example of a $2^{2}$ factorial design is shown in Figure A.2. 


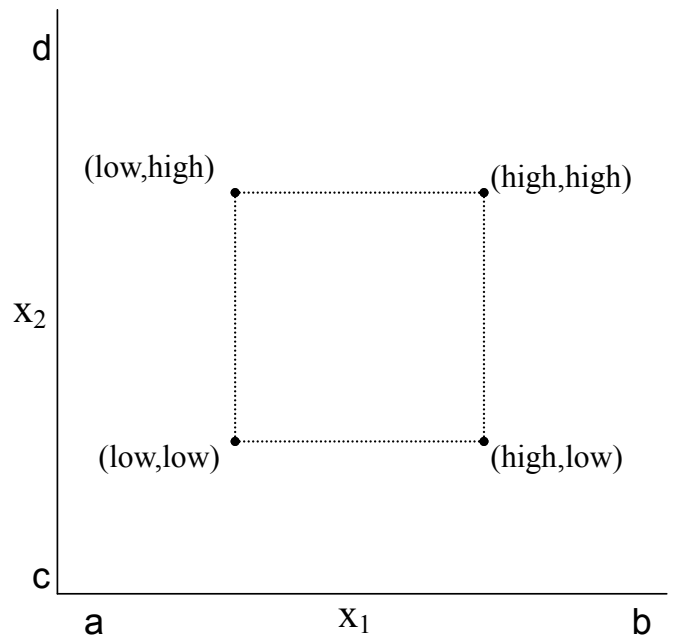

Figure A.2: Example of a $2^{2}$ factorial design.

However, full factorial experimental designs can be expensive, and we might only be able to afford a fractional factorial design, also called a $\boldsymbol{n}^{\boldsymbol{k}-\boldsymbol{j}}$ factorial design, in which only a fraction $\left(n^{k-j}\right)$ of the experimental runs are performed.

\section{A.2 Set Theory}

Definition A.8. A predicate is a description of properties and/or interrelationships of one or more elements (For example, "x is red"). This can be expressed as a function; for example, "isRed $(x)$ " to mean " $x$ is red."

Definition A.9. A set is a collection of objects in which order has no significance. The specification of a set $S$ is the condition that an object must satisfy in order to 
be a member of $S$. A set is defined by its specification as such:

set name $=\{x:$ predicate describing the specification of set that $x$ must satisfy $\}$

Definition A.10. An index set is a set whose elements are used to represent locations.

Definition A.11. A set $R$ is a relation if it is a set of ordered pairs. If $R$ is a relation, $x R y$ means the same thing as $(x, y) \in R$. The domain and range of a relation $R$ (abbreviated dom $R$ and ran $R$ respectively) are defined as

$$
\begin{aligned}
& \operatorname{dom} R=\{x: \text { for some } y, x R y\} \\
& \text { ran } R=\{y: \text { for some } x, x R y\}
\end{aligned}
$$

Definition A.12. Let $R$ be a relation. If $x R x$ for every $x \in \operatorname{dom} R \cup \operatorname{ran} R$, then $R$ is reflexive. If $x R y$ implies $y R x$, then $R$ is symmetric. If $x R y$ and $y R x$ implies $x=y$, then $R$ is antisymmetric. If If $x R y$ and $y R z$ implies $x R z$, then $R$ is transitive.

Definition A.13. Let $R$ be a relation. If $R$ is reflexive, antisymmetric, and transitive, then it is a partial order. A set with a partial order is called a partially ordered set. 
Definition A.14. If $R$ is reflexive, symmetric, and transitive, then it is an equivalence relation. If $x$ Ry for an equivalence relation $R$, then $x$ and $y$ are equivalent under $R$. A partition $\mathcal{C}$ of a set $X$ is a disjoint collection of nonempty subsets of $X$ whose union is $X$. 


\section{Index}

$\begin{array}{ll}\left(i_{\mathscr{F}}\right)^{*}, 49 & \preccurlyeq \omega^{p}, 58 \\ A_{T}, 42 & \Theta_{P}, 56 \\ A_{\tau}, 42 & \delta(i, p, S, d), 52 \\ D, 44 & \operatorname{pref}_{d \mid K}^{p}, 45 \\ D_{p}, 45 & \vec{S}, 43 \\ D_{(i, K)}, 48 & \Phi_{k}^{(i, p)}, 51 \\ D_{(i, K, p)}, 48 & \Upsilon_{k}^{(i, p)}, 51 \\ D_{(i, p)}, 48 & \Lambda_{(i, p)}, 51 \\ I_{\tau}, 42 & \operatorname{pref}_{\tau}^{p}, 43 \\ I_{d}, 44 & \theta_{p}, 56 \\ T, 42 & \theta(i, p), 56 \\ T_{i}, 46 & \omega^{p}, 57 \\ T_{(i, p)}, 47 & \operatorname{cost}(d), 45 \\ X, 42 & l p t(i, S, p), 47 \\ \Omega_{P}, 57 & m p d(i, K, L, p), 48 \\ \Omega_{p}, 57 & n^{k} \text { factorial design, } 215 \\ \preccurlyeq \Omega_{p}, 59 & n^{k-j} \text { factorial design, } 216\end{array}$




\section{INDEX}

targetList ${ }_{\tau}^{p}, 42$

CDEA, 11

affordability criteron, 44

analytical target, 42

analytical task, 42

analytical task specification function, 43

antisymmetric, 217

application criteron, 44

available analytical task list, 46

available design list, 48

axial point, 215

blocking, 213

bounded, 50

center point, 215

central composite design, 214

considerable, 57

considered path, 57

corner point, 215

domain, 217

equivalence relation, 218 equivalent, 218

experimental design, 13,213

experimental design preference ordering function, 45,67

experimental run, 12,213

F-Good, 54

F-Good intermediate index, 53

F-Good terminating index, 53

F-LogErr, 54

F-LogErr intermediate index, 53

F-LogErr terminating index, 53

F-ResErr, 54

F-ResErr intermediate index, 53

F-ResErr terminating index, 53

fact, 31

fact predicate, 31

factorial design, 215

feedback compromise threshold function, 36

feedback evaluation function, 53

Finish, 56

FinishGood, 56 
FinishLogErr, 56

FinishResErr, 56

fractional factorial design, 216

full factorial design, 215

goal, 32

goal command, 32

index priority weight function, 36

index set, 217

initial knowledge state index, 33

intermediate index, 52

knowledge criteron, 42

knowledge state index, 33

knowledge state index set, 33

logical span, 49

lowest priority analytical task list, 47

modeling, 214

most preferred design, 48

nuisance factor, 13,213

operational component, 29 optimization, 214

partial order, 217

partially ordered set, 217

partition, 218

planned move, 30

potential choice, 57

predicate, 216

predictor, 3, 213

preference function, 35

preferred choice, 55, 56

preferred design set, 45

project satisfaction function, 35

range, 217

reducing algorithm, 51

reflexive, 217

relation, 217

research strategic arrangement, 57

research strategy, 30, 56

research strategy set, 57

resource predicate, 32

resource-constrained project scheduling, 
21

response, 3, 213

response function, 214

Response Surface Methodology (RSM), 11

satisfiable, 49

screening, 214

set, 216

specification, 216

specified analytical task list, 47

specified design list, 48

sponsor-designer preference function list,

35

state evaluation function, 54

strategic component, 29

strategic criteron, 44

successor list feedback evaluation func-

tion, 55

successor list, 52

successor potential, 52

symmetric, 217 target list, 42

terminating index, 52,53

transition function, 52

transitive, 217

valid analytical task, 49

valid analytical task set, 50 